B. Janssens and K.-H. Neeb (2015) "Norm Continuous Unitary Representations of Lie Algebras of Smooth Sections,"

International Mathematics Research Notices, Vol. 2015, No. 18, pp. 9081-9137

Advance Access Publication December 1, 2014

doi:10.1093/imrn/rnu231

\title{
Norm Continuous Unitary Representations of Lie Algebras of Smooth Sections
}

\author{
B. Janssens ${ }^{1}$ and K.-H. Neeb ${ }^{2}$ \\ ${ }^{1}$ Mathematisch Instituut, Universiteit Utrecht, Budapestlaan 6, 3584 CD \\ Utrecht, the Netherlands and ${ }^{2}$ Department Mathematik, FAU \\ Erlangen-Nürnberg, Cauerstrasse 11,91058 Erlangen, Germany
}

Correspondence to be sent to: mail@bjadres.nl

Let $\mathfrak{K} \rightarrow X$ be a smooth Lie algebra bundle over a $\sigma$-compact manifold $X$ whose typical fiber is the compact Lie algebra $\mathfrak{k}$. We give a complete description of the irreducible bounded (i.e., norm continuous) unitary representations of the Fréchet-Lie algebra $\Gamma(\mathfrak{K})$ of all smooth sections of $\mathfrak{K}$, and of the LF-Lie algebra $\Gamma_{C}(\mathfrak{K})$ of compactly supported smooth sections. For $\Gamma(\mathfrak{K})$, irreducible bounded unitary representations are finite tensor products of so-called evaluation representations, hence in particular finite dimensional. For $\Gamma_{c}(\mathfrak{K})$, bounded unitary irreducible (factor) representations are possibly infinite tensor products of evaluation representations, which reduces the classification problem to results of Glimm and Powers on irreducible (factor) representations of UHF $C^{*}$-algebras. The key part in our proof is the result that every irreducible bounded unitary representation of a Lie algebra of the form $\mathfrak{k} \otimes_{\mathbb{R}} \mathcal{A}_{\mathbb{R}}$, where $\mathcal{A}_{\mathbb{R}}$ is a unital real complete continuous inverse algebra, is a finite product of evaluation representations. On the group level, our results cover in particular the bounded unitary representations of the identity component $\operatorname{Gau}(P)_{0}$ of the group of smooth gauge transformations of a principal fiber bundle $P \rightarrow X$ with compact base and structure group, and the groups $\operatorname{SU}_{n}(\mathcal{A})_{0}$ with $\mathcal{A}$ a complete involutive commutative continuous inverse algebra.

Received 29 October 2013; Revised 8 October 2014; Accepted 30 October 2014

Communicated by Prof. Dan-Virgil Voiculescu

(C) The Author(s) 2014. Published by Oxford University Press. All rights reserved. For permissions, please e-mail: journals.permissions@oup.com. 


\section{Introduction}

If $G$ is a topological group, then we call a unitary representation $\pi: G \rightarrow \mathrm{U}(\mathcal{H})$ norm continuous or bounded if $\pi$ is continuous with respect to the norm topology on the unitary group $\mathrm{U}(\mathcal{H})$. If $G$ is a Lie group with an exponential function exp: $\mathfrak{g} \rightarrow G$ which is a local diffeomorphism in 0 , that is, $G$ is locally exponential, then basic Lie theory implies that the continuous homomorphisms $\alpha: \mathfrak{g} \rightarrow \mathfrak{u}(\mathcal{H})$, that is, the bounded unitary representations of the Lie algebra $\mathfrak{g}$, are in one-to-one correspondence with the bounded unitary representations of the simply connected covering $\widetilde{G}_{0}$ of the identity component $G_{0}$ of $G$. Here we are using the term "Lie group" for Lie groups modeled on locally convex spaces. For 1-connected, that is, connected and simply connected, locally exponential Lie groups, bounded unitary representations can therefore be understood completely in terms of representations of their Lie algebras.

Let $\mathfrak{K} \rightarrow X$ be a smooth Lie algebra bundle over a $\sigma$-compact smooth finitedimensional manifold $X$ whose typical fiber is the finite-dimensional Lie algebra $\mathfrak{k}$. In the present paper, we shall give a complete description of the irreducible bounded projective unitary representations of the Fréchet-Lie algebra $\Gamma(\mathfrak{K})$ of all smooth sections of $\mathfrak{K}$ (endowed with the smooth compact open topology) and of the LF-Lie algebra $\Gamma_{C}(\mathfrak{K})$ of compactly supported smooth sections, endowed with the locally convex direct limit topology with respect to the Fréchet-Lie algebras $\Gamma(\mathfrak{K})_{C}:=\{s \in \Gamma(\mathfrak{K}): \operatorname{supp}(s) \subseteq C\}$, where $C \subseteq X$ is a compact subset. If $X$ is compact, then both Lie algebras coincide, but if $X$ is non-compact, then their respective bounded representation theory is quite different.

It will be shown that the problem reduces to the case where $\mathfrak{k}$ is compact semisimple, and the representations are linear rather than projective.

For every point $x \in X$ and a unitary representation $(\rho, V)$ of the fiber $\mathfrak{K}_{X} \cong \mathfrak{k}$, we obtain an irreducible unitary representation $\pi_{X, \rho}(s):=\rho(s(x))$ of $\Gamma(\mathfrak{K})$. We call these representations evaluation representations. Our central result is that every irreducible bounded unitary representation of $\Gamma(\mathfrak{K})$ is a finite tensor product of irreducible evaluation representations in different points of $X$ and a one-dimensional representation (of the center). In particular, it is finite dimensional.

If $X$ is non-compact, then the bounded representation theory of the LF-Lie algebra $\Gamma_{C}(\mathfrak{K})$ is "wild" in the sense that there exist bounded factor representations of types II and III. Here our main result is a complete reduction of the classification of bounded irreducible (factor) representations to the classification of irreducible (factor) representations of UHF $C^{*}$-algebras. This correspondence is established as follows. Let 
$\mathbf{x} \subseteq X$ be a locally finite subset and let $\left(\rho_{X}, V_{X}\right)$ be irreducible representations of $\mathcal{K}_{X} \cong \mathfrak{k}$ corresponding to $x \in \mathbf{x}$. We then form the $C^{*}$-algebra

$$
\mathcal{A}_{\mathbf{x}, \rho}:=\widehat{\bigotimes}_{x \in \mathbf{x}} B\left(V_{x}\right)
$$

for which the evaluation representations $\pi_{x, \rho_{x}}$ combine to a continuous morphism $\eta_{\mathbf{x}, \rho}: \Gamma_{C}(\mathfrak{K}) \rightarrow \mathcal{A}_{\mathbf{x}, \rho}$. We then show that a bounded irreducible (factor) representation of $\Gamma_{C}(\mathfrak{K})$ is of the form $\pi=\beta \circ \eta_{\mathbf{x}, \rho}$ for some irreducible (factor) representation of $\beta$ of some $\mathcal{A}_{\mathbf{x}, \rho}$. Conversely, every irreducible (factor) representation $\beta$ of $\mathcal{A}_{\mathbf{x}, \rho}$ defines a bounded irreducible (factor) representation $\beta \circ \eta_{\mathbf{x}, \rho}$ of $\Gamma_{C}(\mathfrak{K})$. Since $\pi$ determines the set $\mathbf{x}$ and the representations $\rho_{X}$ uniquely, we thus obtain a complete reduction of the classification of bounded irreducible (factor) representations of $\Gamma_{C}(\mathfrak{K})$ to the corresponding problem for the UHF $C^{*}$-algebras $\mathcal{A}_{\mathbf{x}, \rho}$. These algebras have been classified by Glimm in [17], where one also finds a characterization of their pure states. Even stronger results were obtained later by Powers in [38], where he shows that the automorphism group acts transitively on the set of pure states, so that every irreducible representation is a twist (by an automorphism) of an infinite tensor product of irreducible representations.

The key part in our proof is the corresponding result for $X$ compact and the trivial Lie algebra bundle, that is, for $\Gamma(\mathfrak{K}) \cong C^{\infty}(X, \mathfrak{k}) \cong \mathfrak{k} \otimes_{\mathbb{R}} C^{\infty}(X, \mathbb{R})$. This is achieved by dealing with a substantially larger class of Lie algebras of the form $\mathfrak{k}_{\mathcal{A}}:=\mathfrak{k} \otimes_{\mathbb{R}} \mathcal{A}_{\mathbb{R}}$, where $\mathcal{A}_{\mathbb{R}}$ is a commutative complete unital continuous inverse algebra, that is, a complete locally convex topological algebra with open unit group and continuous inversion map. Here our main result asserts that every irreducible bounded unitary representation of $\mathfrak{k}_{\mathcal{A}}$ is a finite tensor product of evaluation representations $\pi_{\chi, \rho}(x \otimes a)=\chi(a) \rho(x)$, where $\rho: \mathfrak{k} \rightarrow \mathfrak{u}\left(V_{\rho}\right)$ is an irreducible unitary representation and $\chi: \mathcal{A}_{\mathbb{R}} \rightarrow \mathbb{R}$ is a character. This generalizes a similar result obtained in [34] for the special case $\mathcal{A}_{\mathbb{R}}=C(X, \mathbb{R})$, where $X$ is compact. The main new point here is the passage from Banach algebras to general complete continuous inverse algebras $\mathcal{A}$. This is achieved by a more direct approach that does not rely on holomorphic line bundles and Banach spaces of holomorphic sections.

The methods we use to deal with the Lie algebras of the form $\mathfrak{k}_{\mathcal{A}}$ rely heavily on the fact that $\mathcal{A}_{\mathbb{R}}$ is unital, but it turns out that if $\mathcal{A}_{\mathbb{R}}=C_{0}(X, \mathbb{R})$, where $X$ is a locally compact space countable at infinity, then every bounded unitary representation of $\mathfrak{k}_{\mathcal{A}} \cong C_{0}(X, \mathfrak{k})$ has a unique extension to $C\left(X_{\omega}, \mathfrak{k}\right) \cong C_{0}(X, \mathfrak{k}) \rtimes \mathfrak{k}$, where $X_{\omega}$ is the onepoint compactification of $X$ (cf. Section 6). This extends our classification result to algebras of the form $C_{0}(X, \mathfrak{k})$. 
On the group level our results cover in particular the bounded unitary representations of the identity component $\operatorname{Gau}(P)_{0}$ of the group of smooth gauge transformations of a $K$-principal bundle $P \rightarrow X$, where $K$ is a compact Lie group and $X$ is compact, the groups $\mathrm{SU}_{n}(\mathcal{A})_{0}$, where $\mathcal{A}$ is an involutive complete commutative continuous inverse algebra, and, more generally, connected groups with Lie algebra $\mathfrak{k}_{\mathcal{A}}$.

The structure of the paper is as follows. In Section 1, we start with a key ingredient of our classification for the algebras $\mathfrak{k}_{\mathcal{A}}$, where $\mathcal{A}$ is an involutive commutative complete continuous inverse algebra $\mathcal{A}$. Every function $\phi: \mathcal{A} \rightarrow \mathbb{C}$ which is multiplicative and polynomial of degree $N$ is a product of $N$ algebra homomorphisms $\chi_{j}: \mathcal{A} \rightarrow \mathbb{C}$ (Theorem 1.8). In Section 2, we analyze the bounded unitary representations of $\mathfrak{k}_{\mathcal{A}} \cong \mathfrak{k} \otimes_{\mathbb{R}} \mathcal{A}_{\mathbb{R}}$, where $\mathfrak{k}$ is compact semisimple, $\mathcal{A}_{\mathbb{R}}:=\left\{a \in \mathcal{A}: a^{*}=a\right\}$ and $\mathcal{A}$ is a involutive commutative complete continuous inverse algebra. This is done by studying their complex linear extensions to $\mathfrak{g}(\mathcal{A})=\mathfrak{g} \otimes_{\mathbb{C}} \mathcal{A}$, where $\mathfrak{g}:=\mathfrak{k}_{\mathbb{C}}$ is the complexification of $\mathfrak{k}$. Let $\mathfrak{t} \subseteq \mathfrak{k}$ be maximal abelian, so that $\mathfrak{g}^{0}:=\mathfrak{t} \otimes_{\mathbb{R}} \mathcal{A} \subseteq \mathfrak{g}(\mathcal{A})$ is maximal abelian in $\mathfrak{g}(\mathcal{A})$ and there is a natural triangular decomposition $\mathfrak{g}(\mathcal{A}) \cong \mathfrak{g}^{+} \oplus \mathfrak{g}^{0} \oplus \mathfrak{g}^{-}$determined by a positive system of roots $\Delta^{+}$of the semisimple complex Lie algebra $\mathfrak{g}$. If $\pi: \mathfrak{g}(\mathcal{A}) \rightarrow B(\mathcal{H})$ is a bounded irreducible representation, then the subspace $\mathcal{H}^{\mathfrak{g}^{-}}$is a one-dimensional representation of $\mathfrak{g}^{0}$, hence determined by a linear functional $\lambda: \mathfrak{g}^{0} \rightarrow \mathbb{C}$, called its highest weight. Two such representations are equivalent if and only if their highest weights are equal. Therefore, it remains to determine the set of highest weights. This is achieved in Theorem 2.9, which asserts that these are precisely the sums of functionals of the form $\lambda \otimes \chi$, where $\lambda$ is dominant integral for $\left(\mathfrak{g}, \mathfrak{t}_{\mathbb{C}}, \Delta^{+}\right)$and $\chi: \mathcal{A} \rightarrow \mathbb{C}$ is an involutive algebra homomorphism. Here, the functionals of the form $\lambda \otimes \chi$ correspond to evaluation representations $\pi_{\chi, \lambda}(x \otimes a):=\chi(a) \rho_{\lambda}(x)$, where $\rho_{\lambda}$ is the irreducible representation of $\mathfrak{k}_{\mathbb{C}}$ of highest weight $\lambda$. Part of the proof of Theorem 2.9, namely, the $\mathfrak{s l}_{2}$-reduction needed to show that all highest weights are sums of such functionals, is carried out in Section 3. We obtain in particular that all irreducible bounded unitary representations of $\mathfrak{k}_{\mathcal{A}}$ are finite tensor products of irreducible evaluation representations.

In Section 4, we finally turn to the Lie algebras $\Gamma(\mathfrak{K})$ and $\Gamma_{C}(\mathfrak{K})$. For $\Gamma_{C}(\mathfrak{K})$ an important observation is that every factor representation is a tensor product $\pi=\pi_{1} \otimes \pi_{2}$, where $\pi_{1}$ can be chosen such that it vanishes on the ideal $\Gamma(\mathfrak{K})_{C}$ of sections supported in a given compact subset $C \subseteq X$ and $\pi_{2}$ is a finite tensor product of irreducible evaluation representations. Eventually, this factorization provides the bridge between Lie algebra representations and the infinite tensor products of matrix algebras. In Section 5, we show that assuming compactness of the fiber Lie algebra $\mathfrak{k}$ and linearity of the projective representations does not lead to loss of generality in the classification of 
irreducible bounded projective unitary representations of $\Gamma(\mathfrak{K})$. In Section 6, we first observe that, for $\mathfrak{k}_{\mathcal{A}}$ with $\mathcal{A}$ the non-unital Banach algebra $\mathcal{A}=\ell^{1}(\mathbb{N}, \mathbb{C})$, infinite tensor products of evaluation representations lead to infinite-dimensional irreducible bounded unitary representations and to a wild bounded representation theory. This is in contrast to the fact that, for a locally compact space countable at infinity, all irreducible bounded representations of $C_{0}(X, \mathfrak{k}) \cong \mathfrak{k} \otimes_{\mathbb{R}} C_{0}(X, \mathbb{R})$ are finite tensor products of evaluation representations, even though $\mathcal{A}=C_{0}(X)$ is not unital. If $\mathcal{A}$ is a Banach algebra with $\ell^{1}(\mathbb{N}, \mathbb{C}) \subseteq \mathcal{A} \subseteq C_{0}(\mathbb{N}, \mathbb{C})$, then the representation theory of $\mathfrak{k}_{\mathcal{A}}$ becomes quite involved because some bounded representations of $\ell^{1}(\mathbb{N}, \mathfrak{k})$ only extend to unbounded projective representations of $\mathfrak{k}_{\mathcal{A}}$. We plan to pursue these aspects in the forthcoming paper [26]. In Section 6.3, we assume that $X$ is compact with boundary and in this context we characterize those representations of $\Gamma_{C}\left(X^{\circ}\right)$ extending to the Banach-Lie algebra of $C^{k}$-section whose $k$-jet vanishes on the boundary $\partial X$.

Finite-dimensional representations of Lie algebras of the form $\mathfrak{g} \otimes \mathcal{A}$, where $\mathfrak{g}$ is semisimple and $\mathcal{A}$ a unital commutative algebra, are presently under active investigation from an algebraic point of view. More generally, one studies equivariant map algebras, that is, Lie algebras of the form $(\mathfrak{g} \otimes \mathcal{A})^{\Gamma}$, where $\mathcal{A}$ is the algebra of regular functions on an affine variety and $\Gamma$ a finite group acting on $\mathfrak{g}$ and $\mathcal{A}$. The irreducible finite-dimensional representations of equivariant map algebras have recently been classified by Neher, Savage, and Senesi [36]. Their main result asserts that they are finite tensor products of evaluation representations, which is remarkably parallel to our results in Sections 2 and 4. See also [35] for a recent survey on equivariant map algebras. This connects with our context if $\mathcal{A}=C^{\infty}(Y, \mathbb{C})$ and $\Gamma$ acts freely on $Y$, so that $\mathfrak{K}:=Y \times_{\Gamma} \mathfrak{k}$ is a Lie algebra bundle over $X:=Y / \Gamma$ with $\Gamma(\mathfrak{K}) \cong(\mathfrak{k} \otimes \mathcal{A})^{\Gamma}$.

In [2] an irreducible unitary representation of a Lie group $C_{C}^{\infty}(X, K)$ is called a non-commutative distribution. In this sense, we contribute to the program outlined in [2] by classifying the bounded non-commutative distributions for $X$ and $K$ compact (at least for the identity component). The problem to classify all smooth (projective) irreducible unitary representations of gauge groups is still wide open, although the classification of their central extensions by Janssens and Wockel [27] is a key step towards this goal.

We conclude this introduction with a brief discussion of the literature on unbounded representation of mapping groups. For any, not necessary compact, Lie group $K$, the group $C(X, K)$ has unitary representations obtained as finite tensor products of evaluation representations. However, for some non-compact groups, such as $K=\widetilde{\mathrm{SU}}_{1, n}(\mathbb{C})$, one even has "continuous" tensor product representations which are 
irreducible and extend to groups of measurable maps (cf. [6, 10, 14, 16, 24, 51] for semisimple target groups, [20] for a general discussion and classification results for locally compact target groups, [4] for classification results for compact and nilpotent target groups, and [37] for an example where the target group $U(\infty)$ is infinite dimensional). In the algebraic context of loop groups, these representations also appear in [25] which contains a classification of various types of unitary representations generalizing highest weight representations. All these representations are most naturally defined on groups of measurable maps, so that they neither require a topology nor a smooth structure on $X$.

One of the first references concerning unitary representations of groups of smooth maps such as $C^{\infty}\left(\mathbb{R}, \mathrm{SU}_{2}(\mathbb{C})\right)$ is [15], where the authors introduce the concept of a derivative representation which depends only on the derivatives up to some order $N$ in some point $t_{0} \in \mathbb{R}$. Put in our language, derivative representations are studied as unitary representations of the Lie algebra $\mathfrak{k}_{\mathcal{A}}$, where $\mathcal{A}=\mathbb{C} \llbracket X \rrbracket$ is the continuous inverse algebra of formal power series. In addition to these representations, there exist irreducible representations of mapping groups defined most naturally on groups of Sobolev $H^{1}$-maps, the so-called energy representations (cf. [1-3, 22, 23]). In [23, Sections 15, 17] one even finds some projective modifications of these representations that lift to unitary representations of the simply connected covering group.

For central extensions of loop groups $C^{\infty}\left(\mathbb{S}^{1}, K\right)$, the most studied class of irreducible representations is the class of highest weight representations from Kac-Moody theory which extend to positive energy representations of the semidirect product with $\mathbb{R}$, generating the rotation action on $\mathbb{S}^{1}$ [39]. Other unitary representations are the twisted loop modules from [9]. These are representations of the semidirect product $C^{\infty}\left(\mathbb{S}^{1}, K\right) \rtimes_{\alpha}$ $\mathbb{T}$ induced from finite tensor products of evaluation representations of $C^{\infty}\left(\mathbb{S}^{1}, K\right)$. The restrictions of these representations to the loop group are bounded but not irreducible. In [48] (see also [2, Section 5.4]), Torresani studies projective unitary "highest weight representations" of $C^{\infty}\left(\mathbb{T}^{d}, \mathfrak{k}\right)$, where $\mathfrak{k}$ is compact simple. Besides the finite tensor products of evaluation representations (elementary representations), he finds finite tensor products of evaluation representations of $C^{\infty}\left(\mathbb{T}^{d}, \mathfrak{k}\right) \cong C^{\infty}\left(\mathbb{T}^{d-1}, C^{\infty}(\mathbb{T}, \mathfrak{k})\right)$, where the representations of the target algebra $C^{\infty}(\mathbb{T}, \mathfrak{k})$ are projective highest weight representations (semi-elementary representations).

\section{Notation and terminology}

A (locally convex) Lie group $G$ is a group equipped with a smooth manifold structure modeled on a locally convex space for which the group multiplication and the inversion 
are smooth maps. We write $1 \in G$ for the identity element. Its Lie algebra $\mathfrak{g}=\mathbf{L}(G)$ is identified with the tangent space $T_{1}(G)$. The Lie bracket is obtained by identification with the Lie algebra of left invariant vector fields. A smooth map $\exp _{G}: \mathfrak{g} \rightarrow G$ is called an exponential function if each curve $\gamma_{X}(t):=\exp _{G}(t x)$ is a one-parameter group with $\gamma_{X}^{\prime}(0)=x$. A Lie group $G$ is said to be locally exponential if it has an exponential function for which there is an open 0-neighborhood $U$ in $\mathfrak{g}$ mapped diffeomorphically by $\exp _{G}$ onto an open subset of $G$.

If $\pi: G \rightarrow \mathrm{U}(\mathcal{H})$ is a norm-continuous (=bounded) unitary representation of a locally exponential Lie group $G$, then it is automatically smooth [31, Theorems III.1.5, IV.1.18] and its differential $d \pi: \mathfrak{g} \rightarrow \mathfrak{u}(\mathcal{H})$ is a continuous representation of $\mathfrak{g}$ by skewhermitian bounded operators on $\mathcal{H}$. We call such homomorphisms $\mathfrak{g} \rightarrow \mathfrak{u}(\mathcal{H})$ bounded unitary representations of the Lie algebra $\mathfrak{g}$ because they are representations by bounded operators. For a 1-connected, that is, connected and simply connected, Lie group $G$, the bounded unitary representations of the Lie algebra $\mathfrak{g}$ are in one-to-one correspondence with the unitary representations $\pi: G \rightarrow \mathrm{U}(\mathcal{H})$ which are smooth maps with respect to the Lie group structure on $\mathrm{U}(\mathcal{H})$ defined by the norm. All groups considered in the present paper are locally exponential.

\section{Multiplicative Characters on Continuous Inverse Algebras}

In this section, we provide a key ingredient of our classification results. We show that every function $\phi: \mathcal{A} \rightarrow \mathbb{C}$ on a complete continuous inverse algebra $\mathcal{A}$ which is multiplicative and polynomial of degree $N$ is a product of $N$ algebra homomorphisms $\chi_{j}: \mathcal{A} \rightarrow \mathbb{C}$ (Theorem 1.8). This will be crucial in Section 3 to deal with the Lie algebra $\mathfrak{s u}_{2}(\mathcal{A})$. For unital commutative Banach algebras, this result is already known [34], so the new point is its extension to locally convex algebras.

\subsection{Tensor products of continuous inverse algebras}

\section{Definition 1.1.}

(a) A unital locally convex associative algebra (with continuous multiplication) $\mathcal{A}$ over $\mathbf{k} \in\{\mathbb{R}, \mathbb{C}\}$ is called a continuous inverse algebra (cia for short) if its unit group $\mathcal{A}^{\times}$is open and the inversion map $\mathcal{A}^{\times} \rightarrow \mathcal{A}, a \mapsto a^{-1}$ is continuous. We write $\Gamma_{\mathcal{A}}:=\operatorname{Hom}(\mathcal{A}, \mathbb{C}) \backslash\{0\}$ for the set of non-zero algebra homomorphisms; the characters of $\mathcal{A}$. 
If $\mathcal{A}$ has no unit, then we say that $\mathcal{A}$ is a cia if the unital algebra $\mathcal{A}_{+}:=\mathbf{k} \oplus \mathcal{A}$ with the product

$$
(t, a)(s, b):=(t s, t b+s a+a b)
$$

is a cia.

(b) An involutive cia is a complex cia $\mathcal{A}$, endowed with an antilinear antiisomorphism $a \mapsto a^{*}$. This map is called the involution of $\mathcal{A}$. Then $\mathcal{A}_{\mathbb{R}}:=$ $\left\{a \in \mathcal{A}: a^{*}=a\right\}$ is a real subspace of $\mathcal{A}$ which is a real cia if $\mathcal{A}$ is commutative.

A character $\chi: \mathcal{A} \rightarrow \mathbb{C}$ is said to be involutive if $\chi\left(a^{*}\right)=\overline{\chi(a)}$ holds for $a \in \mathcal{A}$. We write $\Gamma_{\mathcal{A}}^{*}$ for the set of non-zero involutive characters of $\mathcal{A}$. Note that, for any character $\chi$, we obtain another character $\chi^{*}(a):=\overline{\chi\left(a^{*}\right)}$, and that $\chi$ is involutive if and only if $\chi^{*}=\chi$.

\section{Example 1.2.}

(a) For any compact smooth manifold $X$ and $k \in \mathbb{N}_{0} \cup\{\infty\}$, the algebra $\mathcal{A}:=$ $C^{k}(X, \mathbf{k})$ is a unital complete cia. Its characters are of the form $\chi(f)=f(x)$ for some $x \in X$ (cf. [54, Theorem 4.3.1(b)]).

(b) If $X$ is non-compact, then $C^{k}(X, \mathbf{k})$ is no longer a cia because spectra of elements of a cia are compact. In this case, the algebra $\mathcal{A}:=C_{c}^{k}(X, \mathbf{k})$ of compactly supported $C^{k}$-functions is a complete cia (cf. [18, Proposition 7.1]). Its characters are of the form $\chi(f)=f(x)$ for some $x \in X$ (after extending the character to the unital algebra $\mathcal{A}_{+}$, the arguments in the proof of [54, Theorem 4.3.1(b)] carry over).

(c) For $d \in \mathbb{N}$, let $\mathcal{A}:=\mathbf{k} \llbracket X_{1}, \ldots, X_{d} \rrbracket$ denote the algebra of formal power series in the commuting variables $X_{1}, \ldots, X_{d}$. We endow $\mathcal{A}$ with the Fréchet topology defined by the seminorms

$$
p_{\alpha}(a):=\left|a_{\alpha}\right|, \quad \text { where } \quad a=\sum_{\alpha \in \mathbb{N}_{0}^{d}} a_{\alpha} X^{\alpha} .
$$

Then $\mathcal{A}$ is a unital complete cia with unit group $\mathcal{A}^{\times}=\left\{a: a_{0} \neq 0\right\}$ and the unique maximal ideal $\mathfrak{m}=\left(X_{1}, \ldots, X_{d}\right)$ is a hyperplane. Accordingly $\Gamma_{\mathcal{A}}=\{\varepsilon\}$ with $\varepsilon(a)=a_{0}$.

(d) Let $K \subset \mathbb{C}^{n}$ be a compact set. For each open neighborhood $U$ of $K$, we denote by $\mathcal{O}^{\infty}(U)$ the Banach algebra of bounded holomorphic functions on $U$, equipped with the supremum norm. Let $\mathcal{O}_{\text {an }}(K)$ be the algebra of 
germs of holomorphic functions around $K$. Then $\mathcal{O}_{\text {an }}(K)$ is the direct limit of the algebras $\mathcal{O}^{\infty}(U)$, where $U$ runs over the open neighborhoods of $K$, and we equip $\mathcal{O}_{\text {an }}(K)$ with the direct limit topology. This makes $\mathcal{O}_{\text {an }}(K)$ into a complete unital cia (cf. [52]). If $K$ is a rationally convex subset of $\mathbb{C}^{n}$ (meaning that, for every $x \in \mathbb{C}^{n} \backslash K$, there is a polynomial $p$ such that $p(x) \notin p(K)$ ), then every character is of the form $\chi(f)=f(x)$ for some $x \in K$, and $\Gamma_{\mathcal{O}_{\text {an }}(K)}$ is homeomorphic to $K$ (cf. [7]).

(e) The Fréchet algebra $\mathcal{A}:=\mathcal{S}\left(\mathbb{R}^{d}, \mathbb{R}\right)$ of Schwartz functions on $\mathbb{R}^{d}$ is a complete cia with $\Gamma_{\mathcal{A}} \cong \mathbb{R}^{d}$ (all characters are given by evaluations in points of $\mathbb{R}^{d}$ ). This follows from the automatic continuity of characters on cias, the density of $C_{C}^{\infty}\left(\mathbb{R}^{d}, \mathbb{R}\right)$, and (b).

(f) If $\sigma: \Gamma \times X \rightarrow X$ is an action of the group $\Gamma$ by diffeomorphisms on the compact manifold $X$, then the subalgebra $C^{\infty}(X, \mathbf{k})^{\Gamma}$ of $\Gamma$-invariant functions is also a Fréchet cia. More generally, if $\mathcal{A}$ is a unital cia and $\Gamma \subseteq \operatorname{Aut}(\mathcal{A})$, then the subalgebra $\mathcal{A}^{\Gamma}$ of $\Gamma$-fixed points is a cia. Here the main point is to observe that $\mathcal{A}^{\Gamma} \cap \mathcal{A}^{\times} \subseteq\left(\mathcal{A}^{\Gamma}\right)^{\times}$.

Definition 1.3. (a) If $E$ and $F$ are locally convex spaces, then the projective topology on $E \otimes F$ is defined by the seminorms

$$
(p \otimes q)(x)=\inf \left\{\sum_{j=1}^{n} p\left(y_{j}\right) q\left(z_{j}\right): x=\sum_{j=1}^{n} y_{j} \otimes z_{j}\right\}
$$

where $p$ and $q$ are continuous seminorms on $E$ and $F$, respectively (cf. [49, Proposition 43.4]). It has the universal property that continuous bilinear maps $E \times F \rightarrow G, G$ a locally convex space, are in one-to-one correspondence with continuous linear maps $E \otimes F \rightarrow G$. One likewise defines the projective topology for tensor products of finitely many factors.

(b) Now let $\mathcal{A}^{\hat{\otimes} N}$ denote the completed projective $N$-fold tensor product of a commutative cia $\mathcal{A}$. It has the universal property that continuous linear maps $\mathcal{A}^{\hat{\otimes} N} \rightarrow E$ to a complete locally convex space $E$ are in one-to-one correspondence with continuous $N$-linear maps $\mathcal{A}^{N} \rightarrow E$. From the universal property and the associativity of projective tensor products it easily follows that $\mathcal{A}^{\hat{\otimes} N}$ carries a natural unital commutative algebra structure, determined by

$$
\left(a_{1} \otimes \cdots \otimes a_{N}\right)\left(b_{1} \otimes \cdots \otimes b_{N}\right):=a_{1} b_{1} \otimes \cdots \otimes a_{N} b_{N} \quad \text { for } a_{i}, b_{i} \in \mathcal{A} .
$$


The symmetric group $S_{N}$ acts on $\mathcal{A}^{\hat{\otimes} N}$ by permuting the tensor factors, that is,

$$
\sigma\left(a_{1} \otimes \cdots \otimes a_{N}\right):=a_{\sigma^{-1}(1)} \otimes \cdots \otimes a_{\sigma^{-1}(N)}, \quad \sigma \in S_{N}, a_{j} \in \mathcal{A} .
$$

The fixed point algebra $\bar{S}^{N}(\mathcal{A}):=\left(\mathcal{A}^{\hat{\otimes} N}\right)^{S_{N}}$ is also a unital commutative algebra which is a closed subalgebra of $\mathcal{A}^{\hat{\otimes} N}$, hence complete. It is topologically generated by tensors of the form

$$
a_{1} \vee \cdots \vee a_{N}:=\frac{1}{N !} \sum_{\sigma \in S_{N}} a_{\sigma(1)} \otimes \cdots \otimes a_{\sigma(N)},
$$

and by polarization it is actually generated by the diagonal elements

$$
a^{\vee N}=a \vee \cdots \vee a=a \otimes \cdots \otimes a, \quad a \in \mathcal{A} .
$$

We do not know if the (completed) tensor product of two cias is always a cia. The following theorem shows that this is true for complete commutative ones.

Theorem 1.4. If $\mathcal{A}_{1}$ and $\mathcal{A}_{2}$ are complete commutative unital continuous inverse algebras, then their completed projective tensor product $\mathcal{A}_{1} \hat{\otimes} \mathcal{A}_{2}$ is also a continuous inverse algebra.

Proof. Let $\mathcal{B}:=\mathcal{A}_{1} \otimes \mathcal{A}_{2}$ denote the projective tensor product of $\mathcal{A}_{1}$ and $\mathcal{A}_{2}$. Then $\mathcal{B}$ is a unital locally convex commutative algebra. The projective topology on $\mathcal{B}$ is defined by the seminorms $p \otimes q$, where $p$ and $q$ are continuous seminorms on $\mathcal{A}_{1}$ and $\mathcal{A}_{2}$, respectively (cf. Definition 1.3). Suppose that $p$ and $q$ are submultiplicative. For $x=\sum_{j} y_{j} \otimes z_{j}$ and $x^{\prime}=\sum_{j} y_{j}^{\prime} \otimes z_{j}^{\prime}$ we have $x x^{\prime}=\sum_{j, k} y_{j} y_{k}^{\prime} \otimes z_{j} z_{k}^{\prime}$. From

$$
\sum_{j, k} p\left(y_{j} y_{k}^{\prime}\right) q\left(z_{j} z_{k}^{\prime}\right) \leq \sum_{j, k} p\left(y_{j}\right) q\left(z_{j}\right) p\left(y_{k}^{\prime}\right) q\left(z_{k}^{\prime}\right)=\sum_{j} p\left(y_{j}\right) q\left(z_{j}\right) \sum_{k} p\left(y_{k}^{\prime}\right) q\left(z_{k}^{\prime}\right)
$$

we then derive that

$$
(p \otimes q)\left(x x^{\prime}\right) \leq(p \otimes q)(x)(p \otimes q)\left(x^{\prime}\right),
$$

that is, that $p \otimes q$ is submultiplicative.

According to Turpin's Theorem [50], complete commutative continuous inverse algebras have a defining family of submultiplicative seminorms, that is, they are projective limits of commutative Banach algebras. As we have seen above, this property is inherited by $\mathcal{B}$. In particular, $\mathcal{B}$ embeds into a topological product of Banach algebras, which implies that the inversion map $\mathcal{B}^{\times} \rightarrow \mathcal{B}$ is continuous. It therefore remains to show that $\mathcal{B}^{\times}$is a neighborhood of 1 . 
It is clear that $\Gamma_{\mathcal{B}}:=\operatorname{Hom}(\mathcal{B}, \mathbb{C})$ can be identified with the product set $\Gamma_{\mathcal{A}_{1}} \times \Gamma_{\mathcal{A}_{2}}$. As subsets of the dual space $\mathcal{A}_{j}^{\prime}$, the set $\Gamma_{\mathcal{A}_{j}}$ is closed and equicontinuous. Indeed, let $1+U$ be an open neighborhood of 1 in $\mathcal{A}^{\times}$, with $U$ a circular neighborhood of 0 . If $u \in U$, then $1+\gamma u$ is invertible for all $\gamma \in \mathbf{k}$ with $|\gamma| \leq 1$, and thus $\chi(1+\gamma u) \neq 0$ for all $\chi \in \Gamma_{\mathcal{A}}$. This implies that $|\chi(u)|<1$ for all $\chi$, which shows that $\Gamma_{\mathcal{A}}$ is equicontinuous, hence weak-*-compact (cf. [49, Proposition 32.8]). In particular, the Gelfand maps

$$
\mathcal{G}_{j}: \mathcal{A}_{j} \rightarrow C\left(\Gamma_{\mathcal{A}_{j}}\right), \quad \mathcal{G}_{j}(a)(\chi):=\hat{a}(\chi):=\chi(a)
$$

are continuous homomorphisms satisfying $\left\|\mathcal{G}_{j}(u)\right\|<1$ for $u \in U$. We thus obtain on $\Gamma_{\mathcal{B}}$ the structure of a compact Hausdorff space, and the Gelfand map

$$
\mathcal{G}: \mathcal{B} \rightarrow C\left(\Gamma_{\mathcal{B}}\right), \quad a_{1} \otimes a_{2} \mapsto \hat{a}_{1} \otimes \hat{a}_{2}
$$

for $\mathcal{B}$ is continuous by the universal property of the projective tensor product.

We claim that, for $\|\mathcal{G}(b)\|<1$, the Neumann series $\sum_{n=0}^{\infty} b^{n}$ converges in the completion $\hat{\mathcal{B}}$ of $\mathcal{B}$. Let $r: \mathcal{B} \rightarrow \mathbb{R}$ be a submultiplicative seminorm and write $q_{r}: \mathcal{B} \rightarrow \mathcal{B}_{r}$ for the map into the corresponding Banach algebra $\mathcal{B}_{r}$, which is the completion of $\mathcal{B} / r^{-1}(0)$ with respect to the norm induced by $r$. Then $\Gamma_{\mathcal{B}_{r}}$ is a subset of $\Gamma_{\mathcal{B}}$, so that $\|\mathcal{G}(b)\|<1$ implies that $\operatorname{Spec}\left(q_{r}(b)\right.$ ) (which is the image of $\mathcal{G}(b)$ restricted to $\Gamma_{\mathcal{B}_{r}}$ ) is contained in the open unit disc and therefore $\sum_{n=0}^{\infty} q_{r}(b)^{n}$ converges in $\mathcal{B}_{r}$. Since $r$ was arbitrary, it follows that $\sum_{n=0}^{\infty} b^{n}$ converges in $\hat{\mathcal{B}}$, which can be identified with a subset of the product space $\prod_{r} \mathcal{B}_{r}$.

We conclude that $\hat{\mathcal{B}}$ is a commutative unital algebra with an open unit group and continuous inversion. This completes the proof.

Corollary 1.5. For any complete commutative unital continuous inverse algebra $\mathcal{A}$, the completed projective tensor powers $\mathcal{A}^{\hat{\otimes} N}$ are continuous inverse algebras.

\subsection{Multiplicative characters}

We classify the holomorphic multiplicative characters of $\mathcal{A}$. For this, we shall need the following proposition, which is a generalization of the corresponding assertion concerning Banach algebras [34] and which can also be obtained as a special case of [53, Proposition 2.4].

Proposition 1.6. If $\mathcal{A}$ is a complex commutative continuous inverse algebra and $\Gamma \subseteq$ $\operatorname{Aut}(\mathcal{A})$ a finite subgroup, then each character $\chi: \mathcal{A}^{\Gamma} \rightarrow \mathbb{C}$ extends to a character of $\mathcal{A}$. 
Proof. Let $\mathcal{I} \subseteq \mathcal{A}^{\Gamma}$ denote the kernel of $\chi$. Then $\mathcal{I}$ is a proper ideal of $\mathcal{A}^{\Gamma}$, and [34, Lemma 3.1] implies that $\mathcal{A I}$ is a proper ideal of $\mathcal{A}$, hence contained in a maximal ideal $\mathcal{M}$. Now $\mathcal{A} / \mathcal{M}$ is a complex division algebra and a cia [53, Lemma B.9], so that [5] implies that $\mathcal{A} / \mathcal{M} \cong \mathbb{C}$. Therefore, the quotient map $\mathcal{A} \rightarrow \mathcal{A} / \mathcal{M}$ is a character extending $\chi$.

\section{Remark 1.7.}

(a) Unfortunately, the analog of Proposition 1.6 for involutive characters is false. Here is the minimal example. Let $\mathbb{D}:=\mathbb{C}^{2}$ denote the two-dimensional involutive algebra, where the algebra structure is given by pointwise multiplication and $\left(z_{1}, z_{2}\right)^{*}:=\left(\bar{z}_{2}, \bar{z}_{1}\right)$. Then $\Gamma_{\mathbb{D}}=\left\{\chi_{1}, \chi_{2}\right\}$ is a two-element set with $\chi_{j}(z)=z_{j}$ and $\chi_{1}^{*}=\chi_{2}$. In particular, the involutive algebra $\mathbb{D}$ has no involutive characters. However, $\Gamma:=\operatorname{Aut}(\mathbb{D}, *)$ is the two-element group $\{\mathrm{id}, \tau\}$ with $\tau\left(z_{1}, z_{2}\right)=\left(z_{2}, z_{1}\right)$ and $\mathbb{D}^{\Gamma}=\Delta_{\mathbb{C}}$ is the diagonal subalgebra, on which $\chi(z, z):=z$ defines an involutive character.

(b) An involutive cia is called hermitian if, for each hermitian element $a=a^{*}$, the spectrum $\sigma_{\mathcal{A}}(a)$ is contained in $\mathbb{R}$, that is, $a+z \mathbf{l}$ is invertible for $z \notin \mathbb{R}$. For a hermitian cia any character $\chi: \mathcal{A} \rightarrow \mathbb{C}$ is involutive because $\chi(a) \in \mathbb{R}$ for $a=a^{*}$.

(c) The algebra $\mathbb{D}$ is not hermitian because the element $a=(i,-i)$ is hermitian with $\sigma_{\mathbb{D}}(a)=\{ \pm i\}$. Note that $\chi(z):=z_{1} z_{2}$ is a multiplicative character $(\mathbb{D}, \cdot) \rightarrow$ $(\mathbb{C}, \cdot)$ satisfying $\chi\left(a^{*}\right)=\overline{\chi(a)}$ for every $a \in \mathbb{D}$.

Theorem 1.8. Let $\mathcal{A}$ be a commutative unital complete continuous inverse algebra. Then, for every continuous polynomial multiplicative map $\phi: \mathcal{A} \rightarrow(\mathbb{C}, \cdot)$ of degree $N$, there exist finitely many continuous algebra homomorphisms $\chi_{1}, \ldots, \chi_{N}: \mathcal{A} \rightarrow \mathbb{C}$ such that $\varphi=\chi_{1} \cdots \chi_{N}$.

Proof. Write $\phi(a)=\psi(a \vee \cdots \vee a)$ for a continuous linear map $\psi: S^{N}(\mathcal{A}) \rightarrow \mathbb{C}$. For the diagonal generators of $S^{N}(\mathcal{A})$ (cf. Definition 1.3), we then have

$$
\psi\left(a^{\vee N} b^{\vee N}\right)=\psi\left((a b)^{\vee N}\right)=\phi(a b)=\phi(a) \phi(b)=\psi\left(a^{\vee N}\right) \psi\left(b^{\vee N}\right) .
$$

From the linearity of $\psi$ and its multiplicativity on a set of topological linear generators, it now follows that $\psi$ is an algebra homomorphism.

The continuity of $\psi$ further implies that it extends to a continuous linear map on the completion $\bar{S}^{N}(\mathcal{A})$, which is the fixed point algebra for the canonical $S_{N}$-action on the completion of $\mathcal{A}^{\otimes N}$. From Corollary 1.5, we know that this completion is a continuous 
inverse algebra, so that Proposition 1.6 shows that $\psi$ extends to a continuous character $\chi: \mathcal{A}^{\otimes N} \rightarrow \mathbb{C}$. Then

$$
\phi(a)=\chi(a \otimes \cdots \otimes a)=\prod_{i=1}^{n} \chi\left(1^{\otimes i-1} \otimes a \otimes 1^{\otimes n-i}\right)=\chi_{1}(a) \cdots \chi_{N}(a),
$$

where $\chi_{i}: \mathcal{A} \rightarrow \mathbb{C}$ is the character $\chi_{i}(a):=\chi\left(\mathbf{1}^{\otimes i-1} \otimes a \otimes \mathbf{1}^{\otimes n-i}\right)$. Since every character of $\mathcal{A}$ is automatically continuous (because $\mathcal{A}^{\times}$is open), this proves the theorem.

Corollary 1.9. If $\mathcal{A}$ is a complex unital complete cia and $\phi:(\mathcal{A}, \cdot) \rightarrow \mathbb{C}$ holomorphic and multiplicative, then there exist finitely many continuous algebra homomorphisms $\chi_{j}: \mathcal{A} \rightarrow \mathbb{C}$ such that $\varphi=\chi_{1} \cdots \chi_{N}$.

Proof. Since $\phi$ is holomorphic, its restriction to the subalgebra $\mathbb{C} 1$ is holomorphic and multiplicative, hence of the form $\phi(z \mathbf{1})=z^{n}$ for some $n \in \mathbb{N}_{0}$. This implies that $\phi(z a)=z^{n} \phi(a)$ for $z \in \mathbb{C}, a \in \mathcal{A}$. Now $\phi$ has a Taylor expansion in homogeneous polynomials (cf. [31]), and the only nonzero term is the one of degree $n$. Hence, the preceding theorem applies.

Example 1.10. If $X$ is a compact manifold, then $\mathcal{A}=C^{\infty}(X)$ is a unital continuous inverse algebra with spectrum $\Gamma_{\mathcal{A}}=X$ (Example 1.2(b)). As the completed tensor powers are given by $\mathcal{A}^{\hat{\otimes} n} \cong C^{\infty}\left(X^{n}\right)$ [29, Theorem 4] and the $S_{n}$-action on this algebra is induced by the natural action of $S_{n}$ on $X^{n}$, the algebra $S^{n}(\mathcal{A})$ consists of the smooth symmetric functions on $X^{n}$. In particular, its spectrum is the quotient $X^{n} / S_{n}$, which can be identified with the set of $n$-element multisubsets of $X$. As the simple example $X=\mathbb{R}$ already shows, this space is not a smooth manifold.

\section{Irreducible *-Representations of $\mathfrak{g} \otimes \mathcal{A}$}

Let $\mathfrak{k}$ be a compact semisimple Lie algebra and $\mathfrak{g}:=\mathfrak{k}_{\mathbb{C}}$ its complexification. In this section, we develop an analog of the classical Cartan-Weyl theory of highest weight representations for bounded irreducible unitary representations of $\mathfrak{k}_{\mathcal{A}}:=\mathfrak{k} \otimes_{\mathbb{R}} \mathcal{A}_{\mathbb{R}}$. Here and in the remainder of this section, $\mathcal{A}$ is an involutive unital complete commutative continuous inverse algebra. Our main result is a classification of the irreducible bounded unitary representations of $\mathfrak{k}_{\mathcal{A}}$ as finite tensor products of evaluation representations. 


\subsection{Triangular decomposition}

We write $x \mapsto \bar{X}$ for the complex conjugation on $\mathfrak{g}=\mathfrak{k}_{\mathbb{C}}$ and put $x^{*}:=-\bar{X}$. We then have $\mathfrak{k}=\left\{x \in \mathfrak{g}: x^{*}=-x\right\}$. If $\mathfrak{t} \subseteq \mathfrak{k}$ is maximal abelian, then $\mathfrak{h}:=\mathfrak{t}_{\mathbb{C}}$ is a Cartan subalgebra. Let $\Delta \subseteq \mathfrak{h}^{*}$ be the corresponding root system, so that we have the root decomposition

$$
\mathfrak{g}=\mathfrak{h} \oplus \bigoplus_{\alpha \in \Delta} \mathfrak{g}_{\alpha} .
$$

Note that $\overline{\mathfrak{h}}=\mathfrak{h}$ and $\overline{\mathfrak{g}}_{\alpha}=\mathfrak{g}_{-\alpha}$. We write $\check{\alpha} \in \mathfrak{h}$ for the coroot associated to $\alpha \in \Delta$, that is, the unique element $\check{\alpha} \in\left[\mathfrak{g}_{\alpha}, \mathfrak{g}_{-\alpha}\right]$ with $\alpha(\check{\alpha})=2$. Fix a positive system $\Delta^{+}$, and let $\Pi=\left\{\alpha_{1}, \ldots, \alpha_{r}\right\}$ denote the corresponding simple roots.

Then $\mathfrak{g}(\mathcal{A}):=\mathfrak{g} \otimes_{\mathbb{C}} \mathcal{A}$, equipped with Lie bracket

$$
\left[x_{1} \otimes a_{1}, x_{2} \otimes a_{2}\right]:=\left[x_{1}, x_{2}\right] \otimes a_{1} a_{2}
$$

is a locally convex Lie algebra with respect to the natural tensor product topology, for which $\mathfrak{g}(\mathcal{A}) \cong \mathcal{A}^{\text {dimg }}$ as a topological vector space. The antilinear antiautomorphisms $*$ of $\mathfrak{g}$ and $\mathcal{A}$ combine to the antilinear antiautomorphism of $\mathfrak{g}(\mathcal{A})$, defined by

$$
(x \otimes a)^{*}:=x^{*} \otimes a^{*} .
$$

The Lie algebra $\mathfrak{k}_{\mathcal{A}}=\mathfrak{k} \otimes_{\mathbb{R}} \mathcal{A}_{\mathbb{R}}$ is the corresponding real form; $\mathfrak{k}_{\mathcal{A}}=\left\{z \in \mathfrak{g}(\mathcal{A}): z^{*}=-z\right\}$. We define.

$$
\mathfrak{g}^{ \pm}:=\sum_{\alpha \in \mp \Delta^{+}}\left(\mathfrak{g}_{\alpha} \otimes \mathcal{A}\right) \quad \text { and } \quad \mathfrak{g}^{0}:=\mathfrak{h} \otimes \mathcal{A}
$$

to obtain the triangular decomposition

$$
\mathfrak{g}(\mathcal{A})=\mathfrak{g}^{+} \oplus \mathfrak{g}^{0} \oplus \mathfrak{g}^{-} .
$$

(In view of the difference in sign conventions for holomorphic induction and highest weight representations, we define $\mathfrak{g}^{+}$to be the span of the root spaces corresponding to negative roots.)

\subsection{Inducible functionals}

Bounded *-representations correspond to so-called inducible functionals. In this section, we will classify the inducible functionals of $\mathfrak{g}(\mathcal{A})$.

\section{Definition 2.1.}

(a) For a real topological Lie algebra $\mathfrak{u}$, a bounded unitary representation is a pair $(\pi, \mathcal{H})$, where $\mathcal{H}$ is a complex Hilbert space and $\pi: \mathfrak{u} \rightarrow \mathfrak{u}(\mathcal{H})$ a continuous homomorphism of Lie algebras. 
(b) If $(\mathfrak{g}, *)$ is a complex topological Lie algebra and $x \mapsto x^{*}$ a continuous antilinear involutive antiisomorphism, then a bounded $*$-representation of $\mathfrak{g}$ is a pair $(\pi, \mathcal{H})$, where $\mathcal{H}$ is a complex Hilbert space and $\pi: \mathfrak{g} \rightarrow B(\mathcal{H})=\mathfrak{g l}(\mathcal{H})$ a continuous homomorphism of Lie algebras with $\pi\left(x^{*}\right)=\pi(x)^{*}$ for $x \in \mathfrak{g}$. Then the restriction to the real form $\mathfrak{u}:=\left\{x \in \mathfrak{g}: x^{*}=-x\right\}$ is a bounded unitary representation. Conversely, the complex linear extension of every bounded unitary representation of $\mathfrak{u}$ to $\mathfrak{g}$ is a bounded $*$-representation.

Proposition 2.2. Let $(\pi, \mathcal{H})$ be a bounded $*$-representation of $\mathfrak{g}(\mathcal{A})$. Then the following assertions hold:

(i) $\mathcal{E}:=\mathcal{H}^{\mathfrak{g}^{-}}=\left(\pi\left(\mathfrak{g}^{+}\right) \mathcal{H}\right)^{\perp}$ carries a $*$-representation $\rho$ of the commutative subalgebra $\mathfrak{g}^{0}=\mathfrak{h} \otimes \mathcal{A}$.

(ii) $\mathcal{E}$ generates the $\mathfrak{g}(\mathcal{A})$-module $\mathcal{H}$.

(iii) There exists an $N \in \mathbb{N}$ with $\pi\left(\mathfrak{g}^{+}\right)^{N}=\pi\left(\mathfrak{g}^{-}\right)^{N}=\{0\}$.

(iv) The restriction map $R: \pi(\mathfrak{g}(\mathcal{A}))^{\prime} \rightarrow \rho\left(\mathfrak{g}^{0}\right)^{\prime},\left.B \mapsto B\right|_{\mathcal{E}}$ is an isomorphism of von Neumann algebras.

(v) $(\pi, \mathcal{H})$ is irreducible if and only if $\operatorname{dim} \mathcal{E}=1$.

Proof. (i) The relation $\left(\mathfrak{g}^{+}\right)^{*}=\mathfrak{g}^{-}$implies that $\pi\left(\mathfrak{g}^{+}\right)^{*}=\pi\left(\mathfrak{g}^{-}\right)$, which leads to

$$
\mathcal{H}^{\mathfrak{g}^{-}}=\operatorname{ker}\left(\pi\left(\mathfrak{g}^{-}\right)\right)=\left(\pi\left(\mathfrak{g}^{+}\right) \mathcal{H}\right)^{\perp}
$$

Further, $\left[\mathfrak{g}^{0}, \mathfrak{g}^{-}\right] \subseteq \mathfrak{g}^{-}$implies that this closed subspace is invariant under $\mathfrak{g}^{0}$.

(ii) Since, for every $\mathfrak{g}(\mathcal{A})$-invariant subspace $\mathcal{K} \subseteq \mathcal{H}$, the orthogonal complement $\mathcal{K}^{\perp}$ is also invariant, it suffices to show that any non-zero invariant subspace $\mathcal{K}$ intersects $\mathcal{E}$ non-trivially. This amounts to showing that, if $\mathcal{H}$ is non-zero, then $\mathcal{E}$ is non-zero.

To this end, we integrate the representation of the finite-dimensional involutive Lie algebra $\pi: \mathfrak{g} \rightarrow \mathfrak{g l}(\mathcal{H})$ to a holomorphic representation of the corresponding 1-connected group $\pi_{G}: G \rightarrow \operatorname{GL}(\mathcal{H})$. Let $\mathfrak{t}:=\mathfrak{k} \cap \mathfrak{h}$. Then $T:=\exp _{G}(\mathfrak{t})$ is a torus, so that the boundedness of $\pi$ implies that $\mathcal{H}$ decomposes into finitely many $\mathfrak{h}$-weight spaces

$$
\mathcal{H}=\bigoplus_{\beta \in \mathfrak{h}^{*}} \mathcal{H}_{\beta} .
$$

From the relation $\mathfrak{g}_{\alpha}(\mathcal{A}) \mathcal{H}_{\beta} \subseteq \mathcal{H}_{\beta+\alpha}$ and the finiteness of the decomposition of $\mathcal{H}$, we derive the existence of a minimal $N \in \mathbb{N}$ with $\pi\left(\mathfrak{g}^{-}\right)^{N}=\{0\}$. Then $\pi\left(\mathfrak{g}^{-}\right)^{N-1} \mathcal{H}$ is non-zero and contained in $\mathcal{E}$.

(iii) We have already seen that $\pi\left(\mathfrak{g}^{-}\right)^{N}=\{0\}$, so that (ii) follows from $\pi\left(\mathfrak{g}^{+}\right)=\pi\left(\mathfrak{g}^{-}\right)^{*}$. 
(iv) Since $\pi(\mathfrak{g}(\mathcal{A}))^{\prime}$ commutes in particular with $\pi\left(\mathfrak{g}^{-}\right)$, it leaves the subspace $\mathcal{E}$ invariant, so that $R$ is well defined. Since $\mathcal{E}$ generates the $\mathfrak{g}(\mathcal{A})$-module $\mathcal{H}$, the map $R$ is injective. To see that it is surjective, it suffices to show that its range contains all projections of $\rho\left(\mathfrak{g}^{0}\right)^{\prime}$. Here we use that each von Neumann algebra is generated by hermitian projections [12, Chapter 1, Section 1.2] and that images of von Neumann algebras under restriction maps are von Neumann algebras [12, Chaper 1, Section 2.1, Proposition 1]. So let $P=P^{*}=P^{2} \in \rho\left(\mathfrak{g}^{0}\right)^{\prime}$ be a hermitian projection and $\mathcal{E}_{0}:=P(\mathcal{E})$ be its range.

If we denote by $U(\mathfrak{g}(\mathcal{A}))$ the universal enveloping algebra, then $\mathcal{H}_{0}:=\overline{U(\mathfrak{g}(\mathcal{A})) \mathcal{E}_{0}}$ is the closed $\mathfrak{g}(\mathcal{A})$-invariant subspace generated by $\mathcal{E}_{0}$. From $U(\mathfrak{g}(\mathcal{A}))=U\left(\mathfrak{g}^{+}\right) U\left(\mathfrak{g}^{0}\right) U\left(\mathfrak{g}^{-}\right)$, we derive that

$$
\mathcal{H}_{0} \subseteq \overline{U\left(\mathfrak{g}^{+}\right) \mathcal{E}_{0}} \subseteq \mathcal{E}_{0}+\pi\left(\mathfrak{g}^{+}\right) \mathcal{H} \subseteq \mathcal{E}_{0}+\mathcal{E}^{\perp}
$$

which implies that $\mathcal{H}_{0} \cap \mathcal{E}=\mathcal{E}_{0}$. We conclude that the orthogonal projection $\widetilde{P}: \mathcal{H} \rightarrow \mathcal{H}_{0}$, which is contained in $\pi(\mathfrak{g}(\mathcal{A}))^{\prime}$, satisfies $\left.\widetilde{P}\right|_{\mathcal{E}}=P$. Therefore, $R$ is surjective.

(v) In view of Schur's Lemma, $(\pi, \mathcal{H})$ is irreducible if and only if $\pi(\mathfrak{g}(\mathcal{A}))^{\prime}=\mathbb{C} 1$. According to (iv), this is equivalent to the irreducibility of $(\rho, \mathcal{E})$. As $\mathfrak{g}^{0}$ is abelian, this is equivalent to $\operatorname{dim} \mathcal{E}=1$.

Remark 2.3 (Disintegration of bounded representations). For every bounded *-representation $(\pi, \mathcal{H})$ of $\mathfrak{g}(\mathcal{A})$, every irreducible representation of the $C^{*}$-algebra $\mathcal{A}:=$ $C^{*}(\pi(\mathfrak{g}(\mathcal{A})))$ generated by $\pi(\mathfrak{g}(\mathcal{A}))$ defines an irreducible bounded $*$-representations of $\mathfrak{g}(\mathcal{A})$. This implies that $\pi$ is a direct integral of irreducible ones (cf. [11] in the separable case and [21] for inseparable representations). We therefore understand the structure of bounded $*$-representations if we know the irreducible representations.

Definition 2.4. We call an involutive linear functional $\lambda: \mathfrak{g}^{0} \rightarrow \mathbb{C}$ inducible if there exists a bounded $*$-representation $(\pi, \mathcal{H})$ of $\mathfrak{g}(\mathcal{A})$ with $(\rho, \mathcal{E}) \cong(\lambda, \mathbb{C})$, that is, if $\lambda$ occurs as the $\mathfrak{g}^{0}$-weight on $\mathcal{E}$ for some irreducible bounded representation of $\mathfrak{g}(\mathcal{A})$.

Lemma 2.5. If $\left(\pi_{1}, \mathcal{H}_{1}\right)$ and $\left(\pi_{2}, \mathcal{H}_{2}\right)$ are irreducible representations with $\mathfrak{g}^{0}$-weights $\lambda_{1}$ and $\lambda_{2}$ on $\mathcal{E}$, then $\pi_{1} \cong \pi_{2}$ if and only if $\lambda_{1}=\lambda_{2}$.

Proof. Suppose that $\lambda_{1}=\lambda_{2}=\lambda$. Consider the direct sum representation $\pi:=\pi_{1} \oplus \pi_{2}$ on $\mathcal{H}:=\mathcal{H}_{1} \oplus \mathcal{H}_{2}$. Then $\mathcal{E}=\mathcal{E}_{1} \oplus \mathcal{E}_{2}$ is two-dimensional with $\rho(x)(v, w)=(\lambda(x) v, \lambda(x) w)$. In particular, we obtain $\rho\left(\mathfrak{g}^{0}\right)^{\prime} \cong M_{2}(\mathbb{C})$ for the commutant. We conclude that $\pi(\mathfrak{g}(\mathcal{A}))^{\prime} \cong$ $M_{2}(\mathbb{C})$ (Proposition 2.2(iv)). If the representations $\pi_{1}$ and $\pi_{2}$ were not equivalent, then we would have obtained $\pi(\mathfrak{g}(\mathcal{A}))^{\prime} \cong \mathbb{C}^{2}$ by Schur's Lemma. This shows that $\pi_{1} \cong \pi_{2}$. 
Conversely, suppose that $\pi_{1} \simeq \pi_{2}$. Then the intertwiner $U: \mathcal{H}_{1} \rightarrow \mathcal{H}_{2}$ restricts to an intertwiner $\left.U\right|_{\mathcal{E}_{1}}: \mathcal{E}_{1} \rightarrow \mathcal{E}_{2}$ of the one-dimensional representations $\rho_{1}$ and $\rho_{2}$, so that $\lambda_{1}=\lambda_{2}$.

Definition 2.6. In view of the preceding lemma, we write $\left(\pi_{\lambda}, \mathcal{H}_{\lambda}\right)$ for the unique irreducible representation with $(\rho, \mathcal{E}) \cong(\lambda, \mathbb{C})$. We call $\lambda$ the highest weight of $\pi_{\lambda}$.

Lemma 2.7. If $\lambda$ and $\mu$ are inducible, then so is their sum $\lambda+\mu$.

Proof. We consider the $*$-representation $(\pi, \mathcal{H})$ with

$$
\mathcal{H}:=\mathcal{H}_{\lambda} \otimes \mathcal{H}_{\mu} \quad \text { and } \quad \pi:=\pi_{\lambda} \otimes 1+1 \otimes \pi_{\mu}
$$

Then $\mathcal{F}:=\mathcal{E}_{\lambda} \otimes \mathcal{E}_{\mu} \subseteq \mathcal{E}$ is a one-dimensional subspace on which $\mathfrak{g}^{0}$ acts by the weight $\lambda+$ $\mu$. Since $\mathcal{H}_{0}:=\overline{U(\mathfrak{g}(\mathcal{A})) \mathcal{F}} \subseteq \mathcal{H}$ is a $\mathfrak{g}(\mathcal{A})$-submodule with $\mathcal{F}=\left(\mathcal{H}_{0}\right)^{\mathfrak{g}^{-}}$(Proposition 2.2(iv)), it carries an irreducible representation with highest weight $\lambda+\mu$.

Definition 2.8. Let $\mathcal{A}$ be a commutative involutive cia and $\chi: \mathcal{A} \rightarrow \mathbb{C}$ an involutive character. Then $\operatorname{ev}_{\chi}:=\mathrm{id} \otimes \chi: \mathfrak{g}(\mathcal{A}) \rightarrow \mathfrak{g}(\mathbb{C}) \cong \mathfrak{g}$ is an involutive algebra homomorphism. If $(\rho, \mathcal{H})$ is a $*$-representation of $\mathfrak{g}$ with highest weight $\lambda$, then the representation $\pi_{\chi, \rho}:=$ $\rho \circ \mathrm{ev}_{\chi}$ of $\mathfrak{g}(\mathcal{A})$ on $\mathcal{H}$ is called an irreducible evaluation representation. This is an irreducible $*$-representation with highest weight $\lambda \otimes \chi$.

The proof of the following theorem builds on the main result of Section 3 (Theorem 3.4) which deals with the special case $\mathfrak{g}=\mathfrak{s l}_{2}(\mathbb{C})$.

Theorem 2.9. All functionals of the form $\lambda \otimes \chi$, where $\chi \in \Gamma_{\mathcal{A}}^{*}$ is an involutive character and $\lambda \in \mathfrak{h}^{*}$ is dominant integral, that is, $\lambda(\check{\alpha}) \in \mathbb{N}_{0}$ for $\alpha \in \Pi$, are inducible. Conversely, any inducible functional is such a finite sum.

Proof. The definition of the evaluation representations shows that any functional of the form $\lambda \otimes \chi, \chi \in \Gamma_{\mathcal{A}}^{*}$ and $\lambda \in \mathfrak{h}^{*}$ dominant integral, is inducible. Further, Lemma 2.7 implies that any sum of such functionals is inducible.

We now show that any inducible functional is of this form. Suppose that $\lambda$ is inducible and that $(\pi, \mathcal{H}):=\left(\pi_{\lambda}, \mathcal{H}_{\lambda}\right)$ is the corresponding representation of $\mathfrak{g}(\mathcal{A})$. Let $\alpha \in \Delta$ and

$$
\mathfrak{g}^{\alpha}(\mathcal{A}):=\mathfrak{g}_{\alpha}(\mathcal{A})+\mathfrak{g}_{-\alpha}(\mathcal{A})+\check{\alpha} \otimes \mathcal{A} \cong \mathfrak{s l}_{2}(\mathcal{A})
$$

Then $\mathcal{E}$ is annihilated by $\mathfrak{g}_{\alpha}(\mathcal{A})$ and generates a $\mathfrak{g}^{\alpha}(\mathcal{A})$-subrepresentation $\left(\pi_{\alpha}, \mathcal{H}_{\alpha}\right)$. Then

$$
U\left(\mathfrak{g}^{\alpha}(\mathcal{A})\right) \mathcal{E}=U\left(\mathfrak{g}_{-\alpha}(\mathcal{A})\right) \mathcal{E} \subseteq \mathcal{E}+\pi_{\alpha}\left(\mathfrak{g}_{-\alpha}(\mathcal{A})\right) \mathcal{H}_{\alpha}
$$


implies that

$$
\mathcal{E}_{\alpha}:=\mathcal{H}_{\alpha}^{\mathfrak{g}_{\alpha}(\mathcal{A})}=\left(\pi_{\alpha}\left(\mathfrak{g}_{-\alpha}(\mathcal{A})\right) \mathcal{H}_{\alpha}\right)^{\perp}=\mathcal{E}
$$

is one-dimensional, so that $\left(\pi_{\alpha}, \mathcal{H}_{\alpha}\right)$ is irreducible with $\mathcal{E}_{\alpha}=\mathcal{E}$. Therefore, $\left.\lambda\right|_{\check{\alpha} \otimes \mathcal{A}}$ is inducible. The main result of Section 3 (Theorem 3.4) asserts that there exist finitely many pairwise different involutive characters $\chi_{1}, \ldots, \chi_{N} \in \Gamma_{\mathcal{A}}$ and $m_{j} \in \mathbb{N}_{0}$ with

$$
\lambda(\check{\alpha} \otimes a)=\sum_{j=1}^{N} m_{j} \chi_{j}(a) \quad \text { for } a \in \mathcal{A} \text {. }
$$

Recall that $\Pi=\left\{\alpha_{1}, \ldots, \alpha_{r}\right\}$ is the set of simple roots. Then $\check{\alpha}_{1}, \ldots, \check{\alpha}_{r}$ is a basis of $\mathfrak{h}$, and we obtain finitely many pairwise different involutive characters $\chi_{j} \in \Gamma_{\mathcal{A}}$ and $m_{i j} \in \mathbb{N}_{0}$ with

$$
\lambda\left(\check{\alpha}_{i} \otimes a\right)=\sum_{j} m_{i j} \chi_{j}(a) \quad \text { for } a \in \mathcal{A} .
$$

Define $\lambda_{j} \in \mathfrak{h}^{*}$ by $\lambda_{j}\left(\check{\alpha}_{i}\right)=m_{i j}$ and note that $\lambda_{j}$ is dominant integral. We now have for each $i$

$$
\lambda\left(\check{\alpha}_{i} \otimes a\right)=\sum_{j}\left(\lambda_{j} \otimes \chi_{j}\right)\left(\check{\alpha}_{i} \otimes a\right) \quad \text { for } a \in \mathcal{A},
$$

so that $\lambda=\sum_{j} \lambda_{j} \otimes \chi_{j}$.

\subsection{Bounded *-representations}

The classification of inducible functionals now yields the irreducible $*$-representations of $\mathfrak{g}(\mathcal{A})$.

Lemma 2.10. If $\chi_{1}, \ldots, \chi_{N}: \mathcal{A} \rightarrow \mathbb{C}$ are mutually different characters of the complex algebra $\mathcal{A}$, then the homomorphism

$$
\chi: \mathcal{A} \rightarrow \mathbb{C}^{N}, \quad \chi(a):=\left(\chi_{1}(a), \ldots, \chi_{N}(a)\right)
$$

is surjective.

Proof. This follows immediately from the fact that characters are linearly independent.

Theorem 2.11. Every bounded irreducible $*$-representation $(\pi, \mathcal{H})$ of $\mathfrak{g}(\mathcal{A})$ is unitarily equivalent to a finite tensor product of irreducible evaluation representations; there exists a finite set $\mathbf{x} \subseteq \Gamma_{\mathcal{A}}^{*}$ of involutive characters, and for each $\chi \in \mathbf{x}$ an 
irreducible $*$-representation $\rho_{\chi}$ of $\mathfrak{g}$, such that $\pi \simeq \pi_{\mathbf{x}, \rho}:=\bigotimes_{\chi \in \mathbf{x}} \rho_{\chi} \circ \mathrm{ev}_{\chi}$. Conversely, all such representations are irreducible, and $\pi_{\mathbf{x}, \rho} \simeq \pi_{\mathbf{x}^{\prime}, \rho^{\prime}}$ if and only if $\mathbf{x}=\mathbf{x}^{\prime}$ and $\rho_{\chi} \simeq \rho_{\chi}^{\prime}$ for all $\chi \in \mathbf{x}$.

Proof. In view of Theorem 2.9, we can write the highest weight $\lambda: \mathfrak{h} \otimes \mathcal{A} \rightarrow \mathbb{C}$ of $(\pi, \mathcal{H})$ in the form $\lambda=\sum_{\chi \in \mathbf{x}} \lambda_{\chi} \otimes \chi$, where the $\lambda_{\chi}$ are dominant weights and $\mathbf{x} \subseteq \Gamma_{\mathcal{A}}$ is a finite set of involutive characters of $\mathcal{A}$. Then

$$
\mathrm{ev}_{\mathbf{x}}: \mathfrak{g}(\mathcal{A}) \rightarrow \mathfrak{g}^{\mathrm{x}}, \quad \mathrm{ev}(x \otimes a)(\chi)=\chi(a) X
$$

is a surjective homomorphism of Lie algebras (Lemma 2.10) through which all the evaluation representations $\pi_{\chi, \rho_{\chi}}$ factor, where $\rho_{\chi}$ is the representation with highest weight $\lambda_{\chi}$. This implies that the tensor product $\pi_{\mathbf{x}, \rho}=\bigotimes_{\chi \in \mathbf{x}} \rho_{\chi} \circ \mathrm{ev}_{\chi}$ defines an irreducible representation of $\mathfrak{g}(\mathcal{A})$, which clearly has highest weight $\lambda$.

Conversely, suppose that $\pi_{\mathbf{x}, \rho} \simeq \pi_{\mathbf{x}^{\prime}, \rho^{\prime}}$. Then $\operatorname{ker} \chi^{\prime} \supset \bigcap_{\chi \in \mathbf{x}} \operatorname{ker} \chi$ for all $\chi^{\prime} \in \mathbf{x}^{\prime}$, so that $\chi^{\prime} \in \mathbf{x}$ by Lemma 2.10. Similarly, we have $\chi \in \mathbf{x}^{\prime}$ for all $\chi \in \mathbf{x}$, whence $\mathbf{x}^{\prime}=\mathbf{x}$. It then follows from Lemma 2.5 and the surjectivity of $\mathrm{ev}_{\mathbf{x}}$ that $\pi_{\chi} \simeq \pi_{\chi}^{\prime}$ for all $\chi \in \mathbf{x}$.

Specializing to $\mathcal{A}=C^{\infty}(X, \mathbb{C})$ if $X$ is a compact manifold (Example 1.2(a)) and to $\mathcal{A}=C_{C}^{\infty}(X, \mathbb{C})_{+}$if $X$ is a non-compact manifold (Example 1.2(b)), we notice that $\Gamma_{\mathcal{A}} \simeq X$, so that we obtain:

Corollary 2.12. Let $X$ be a smooth manifold. Then every bounded irreducible *-representation $(\pi, \mathcal{H})$ of the Fréchet-Lie algebra $C^{\infty}(X, \mathfrak{k})$ (if $X$ is compact) or $C_{C}^{\infty}(X, \mathfrak{k}) \rtimes \mathfrak{k} \cong \mathfrak{k} \otimes_{\mathbb{R}} C_{C}^{\infty}(X, \mathbb{R})_{+}$(if $X$ is non-compact) is a finite tensor product $\pi \cong$ $\bigotimes_{X \in \mathbf{X}} \rho_{X} \circ \mathrm{ev}_{X}$ of irreducible evaluation representations for some finite subset $\mathbf{x} \subseteq X$ and irreducible $*$-representations $\rho_{X}$ of $\mathfrak{g}$. Conversely, all such representations are irreducible, and $\pi_{\mathbf{x}, \rho} \simeq \pi_{\mathbf{x}^{\prime}, \rho^{\prime}}$ if and only if $\mathbf{x}=\mathbf{x}^{\prime}$ and $\rho_{X} \simeq \rho_{X}^{\prime}$ for all $x \in \mathbf{x}$.

\subsection{Translation to the group context}

Theorem 2.11 classifies the bounded irreducible $*$-representations of $\mathfrak{g}(\mathcal{A})$. We now discuss how these results lift to the group level, providing a complete classification of the bounded unitary representations of the 1-connected Lie group $K_{\mathcal{A}}$ with Lie algebra $\mathfrak{k} \otimes_{\mathbb{R}} \mathcal{A}_{\mathbb{R}}$. Here and throughout this section, $\mathcal{A}$ will be a unital commutative involutive complete cia and $\mathfrak{k}$ a compact semisimple Lie algebra. 


\subsubsection{Matrix groups over cias}

We start by introducing some Lie groups related to $\mathcal{A}$.

\section{Definition 2.13.}

(a) Since $\mathcal{A}$ is commutative, $\operatorname{tr}: \mathfrak{g l}_{n}(\mathcal{A}) \rightarrow \mathcal{A},\left(x_{i j}\right) \mapsto \sum_{j=1}^{n} x_{j j}$ is a homomorphism of Lie algebras, so that

$$
\mathfrak{s l}_{n}(\mathcal{A}):=\left\{x \in \mathfrak{g l}_{n}(\mathcal{A}): \operatorname{tr} x=0\right\}
$$

is a closed ideal which is the Lie algebra of the Lie subgroup

$$
\mathrm{SL}_{n}(\mathcal{A}):=\operatorname{ker}(\operatorname{det})
$$

where det: $\mathrm{GL}_{n}(\mathcal{A}) \rightarrow \mathcal{A}^{\times}$is the natural determinant homomorphism (cf. [31, Proposition IV.3.4]). We write $\widetilde{\mathrm{SL}}_{n}(\mathcal{A})$ for the unique 1-connected locally exponential Lie group with Lie algebra $\mathfrak{s l}_{n}(\mathcal{A})$, which is the simply connected covering of the identity component $\operatorname{SL}_{n}(\mathcal{A})_{0}$.

(b) If $\mathcal{A}$ is involutive, then the involution extends to all matrix algebras $M_{n}(\mathcal{A})$ by

$$
\left(x_{i j}\right)^{*}:=\left(x_{j i}^{*}\right)
$$

We have corresponding unitary groups and their Lie algebras

$$
\mathrm{U}_{n}(\mathcal{A}):=\left\{g \in \mathrm{GL}_{n}(\mathcal{A}): g^{*}=g^{-1}\right\} \quad \text { and } \quad \mathfrak{u}_{n}(\mathcal{A}):=\left\{x \in \mathfrak{g l}_{n}(\mathcal{A}): x^{*}=-x\right\} .
$$

The closed subalgebra $\mathfrak{s u}_{n}(\mathcal{A}):=\mathfrak{u}_{n}(\mathcal{A}) \cap \mathfrak{s l}_{n}(\mathcal{A})$ is the Lie algebra of the Lie subgroup

$$
\mathrm{SU}_{n}(\mathcal{A}):=\mathrm{U}_{n}(\mathcal{A}) \cap \mathrm{SL}_{n}(\mathcal{A})
$$

Hence, it is the Lie algebra of a unique 1-connected Lie group, denoted $\widetilde{\mathrm{SU}}_{n}(\mathcal{A})$.

\section{Remark 2.14.}

(a) Let $K$ be a 1-connected compact Lie group and $G:=K_{\mathbb{C}}$ its universal complexification. We write $\mathfrak{k} \subseteq \mathfrak{g}=\mathfrak{k}_{\mathbb{C}}$ for their Lie algebras. Then $\mathfrak{k}_{\mathcal{A}}=\mathfrak{k} \otimes_{\mathbb{R}} \mathcal{A}_{\mathbb{R}}$ is a real form of the complex Lie algebra $\mathfrak{g}(\mathcal{A})=\mathfrak{g} \otimes_{\mathbb{C}} \mathcal{A}$. Let $K_{\mathcal{A}}$, resp., $G(\mathcal{A})$ be corresponding 1-connected locally exponential Lie groups. Then the canonical morphism $\eta: K_{\mathcal{A}} \rightarrow G(\mathcal{A})$ for which $\mathrm{L}(\eta)$ is the inclusion $\mathfrak{k} \otimes_{\mathbb{R}} \mathcal{A}_{\mathbb{R}} \hookrightarrow \mathfrak{g} \otimes_{\mathbb{C}} \mathcal{A}$ 
has the following universal property. For each smooth morphism $\alpha: K_{\mathcal{A}} \rightarrow H$, where $H$ is a complex Lie group with exponential function, there exists a unique holomorphic morphism $\alpha_{\mathbb{C}}: G(\mathcal{A}) \rightarrow H$ with $\alpha_{\mathbb{C}} \circ \eta=\alpha$. To verify this claim, we simply have to integrate the complex linear extension $\mathbf{L}(\alpha)_{\mathbb{C}}: \mathfrak{g} \otimes$ $\mathcal{A} \rightarrow \mathbf{L}(H)$ to a group homomorphism [31, Theorem 4.1.19].

(b) For $\mathfrak{k}=\mathfrak{s u}_{n}(\mathbb{C})$ and $\mathfrak{g}=\mathfrak{s l}_{n}(\mathbb{C})$ we have $K_{\mathcal{A}}=\widetilde{\mathrm{SU}}_{n}(\mathcal{A})$ and $G(\mathcal{A})=\widetilde{\mathrm{SL}}_{n}(\mathcal{A})$.

\subsubsection{Bounded unitary representations}

We now discuss the translation from Lie algebra to Lie group representations.

Definition 2.15. Let $G$ be a complex locally exponential Lie group endowed with an antiholomorphic antiautomorphism $g \mapsto g^{*}$. A holomorphic *-representation of $G$ is a pair $(\pi, \mathcal{H})$, where $\mathcal{H}$ is a complex Hilbert space and $\pi: G \rightarrow G L(\mathcal{H})$ is a holomorphic homomorphism satisfying $\pi\left(g^{*}\right)=\pi(g)^{*}$ for $g \in G$. Then $d \pi: \mathfrak{g} \rightarrow B(\mathcal{H})$ is a bounded *-representation of $\mathfrak{g}$ in the sense of Definition 2.1 .

With Remark 2.14, we immediately obtain the following generalization of Weyl's correspondence between unitary representations of $K_{\mathcal{A}}$ and holomorphic representations of $G(\mathcal{A})$.

Proposition 2.16. Let $\mathcal{A}$ be a commutative involutive cia, $\mathfrak{g}$ a semisimple complex Lie algebra with compact real form $\mathfrak{k}, G(\mathcal{A})$ a 1-connected Lie group with Lie algebra $\mathfrak{g} \otimes_{\mathbb{C}} \mathcal{A}$, and $K_{\mathcal{A}}$ a 1 -connected Lie group with Lie algebra $\mathfrak{k} \otimes_{\mathbb{R}} \mathcal{A}_{\mathbb{R}}$.

If $\left(\pi_{\mathbb{C}}, \mathcal{H}\right)$ is a holomorphic $*$-representation of $G(\mathcal{A})$, then $\pi:=\pi_{\mathbb{C}} \circ \eta$ is a bounded unitary representation of $K_{\mathcal{A}}$. We thus obtain a one-to-one correspondence between holomorphic $*$-representations $\pi_{\mathbb{C}}$ of $G(\mathcal{A})$ and bounded unitary representations $\pi$ of $K_{\mathcal{A}}$.

The commutants $\pi\left(K_{\mathcal{A}}\right)^{\prime}$ and $\pi_{\mathbb{C}}(G(\mathcal{A}))^{\prime}$ coincide, so that $\pi$ is irreducible if and only if $\pi_{\mathbb{C}}$ has this property.

Combining Theorem 2.11 with the preceding proposition, we obtain:

Theorem 2.17. Let $\mathcal{A}$ be a commutative involutive complete cia. Then every bounded irreducible unitary representation $(\pi, \mathcal{H})$ of $K_{\mathcal{A}}$ is a finite tensor product of evaluation representations corresponding to irreducible representations of $K$. In particular, $\mathcal{H}$ is finite dimensional. 
Remark 2.18. Let $K$ be a compact Lie group with Lie algebra k. Then Tychonov's Theorem implies that the product group $K^{X}$ of all maps $X \rightarrow K$ is a compact group. Any irreducible continuous unitary representation $(\pi, \mathcal{H})$ of $K^{X}$ is finite dimensional and factors through a projection to some quotient Lie group $K^{\mathbf{x}}$, where $\mathbf{x} \subseteq X$ is a finite subset. Hence, there exist irreducible unitary representations $\left(\rho_{X}, V_{X}\right)$, labeled by $x \in \mathbf{x}$, such that $\pi(g)=\bigotimes_{x \in \mathbf{x}} \rho_{X}\left(g_{X}\right)$.

It now follows from Corollary 2.12 that every irreducible bounded unitary representation of the connected Lie group $C^{\infty}(X, K)_{0}$, where $X$ is a compact manifold, extends to a continuous representation of the compact group $K^{X}$. This observation may be of some interest in the context of Loop Quantum Gravity where one works with compactified gauge groups of the form $K^{X}$ (cf. [47]). As we shall see in Section 4, this picture changes for the group $C_{C}^{\infty}(X, K)$ when $X$ is a non-compact manifold. In this case, there exist bounded irreducible unitary representations that do not extend to the compact group $K^{X}$. However, it turns out that they all factor through representations of groups of the type $K^{(\mathbf{x})}$ with $\mathbf{x} \subset X$ a locally finite subset.

\section{Irreducible *-Representations of $\mathfrak{s l}_{2}(\mathcal{A})$}

In this section, we apply Theorem 1.8 on multiplicative characters to obtain a classification of the inducible functionals of $\mathfrak{s l}_{2}(\mathcal{A})$, where $\mathcal{A}$ is a complete unital involutive commutative cia. We have already seen how this was used in Theorem 2.9 to obtain the corresponding result for tensor products $\mathfrak{g}(\mathcal{A})=\mathfrak{k}_{\mathbb{C}} \otimes \mathcal{A}$, where $\mathfrak{k}$ is a compact Lie algebra and $\mathfrak{g}=\mathfrak{k}_{\mathbb{C}}$. Theorem 2.9 in turn led to the characterization of bounded irreducible *-representations in Theorem 2.11.

Throughout this section, $\mathcal{A}$ will be a unital commutative involutive complete cia.

\subsection{The group $\widetilde{\mathrm{SL}}_{2}(\mathcal{A})$}

We use the standard notation for the basis elements of $\mathfrak{s l}_{2}(\mathbb{C})$ :

$$
h:=\left(\begin{array}{cc}
1 & 0 \\
0 & -1
\end{array}\right), \quad e:=\left(\begin{array}{ll}
0 & 1 \\
0 & 0
\end{array}\right) \quad \text { and } \quad f:=\left(\begin{array}{ll}
0 & 0 \\
1 & 0
\end{array}\right)
$$

satisfying the relations $[h, e]=2 e,[h, f]=-2 f$ and $[e, f]=h$. In the notation of Section 2, we have $\mathfrak{g}=\mathfrak{g}^{+} \oplus \mathfrak{g}^{0} \oplus \mathfrak{g}^{-}$with

$$
\mathfrak{g}=\mathfrak{s l}_{2}(\mathbb{C}), \quad \mathfrak{g}^{0}=\mathcal{A} h, \quad \mathfrak{g}^{-}=\mathcal{A} e, \quad \text { and } \quad \mathfrak{g}^{+}=\mathcal{A} f
$$


Recall that $\mathfrak{g}^{+}$is the span of the negative roots. Using Gauss decomposition, we see that the identity component $\mathrm{SL}_{2}(\mathcal{A})_{0}$ of the Lie group $\mathrm{SL}_{2}(\mathcal{A})$ is generated by matrices of the form

$$
\left(\begin{array}{ll}
1 & a \\
0 & 1
\end{array}\right), \quad\left(\begin{array}{ll}
1 & 0 \\
b & 1
\end{array}\right), \quad a, b \in \mathcal{A}
$$

Indeed, this follows from

$$
\left(\begin{array}{ll}
a & b \\
c & d
\end{array}\right) \in \mathrm{e}^{\mathcal{A f}} \Delta(a) \mathrm{e}^{\mathcal{A} e} \quad \text { for } \quad \Delta(a):=\left(\begin{array}{cc}
a & 0 \\
0 & a^{-1}
\end{array}\right) \text { and } a \in \mathcal{A}^{\times}
$$

and

$$
\Delta(a)=\left(\begin{array}{cc}
a & 0 \\
0 & a^{-1}
\end{array}\right)=\left(\begin{array}{cc}
1 & 0 \\
a^{-1}-1 & 1
\end{array}\right)\left(\begin{array}{ll}
1 & 1 \\
0 & 1
\end{array}\right)\left(\begin{array}{cc}
1 & 0 \\
a-1 & 1
\end{array}\right)\left(\begin{array}{cc}
1 & -a^{-1} \\
0 & 1
\end{array}\right) \text { for } a \in \mathcal{A}^{\times} .
$$

We write $q: \widetilde{\mathrm{SL}}_{2}(\mathcal{A}) \rightarrow \mathrm{SL}_{2}(\mathcal{A})_{0}$ for the universal covering homomorphism with $\mathrm{L}(q)=$ $\operatorname{id}_{\mathfrak{s} l_{2}(\mathcal{A})}$ and $e_{12}:(\mathcal{A},+) \rightarrow \widetilde{\mathrm{SL}}_{2}(\mathcal{A})$ for the unique continuous homomorphism satisfying

$$
q\left(e_{12}(x)\right)=\left(\begin{array}{ll}
1 & x \\
0 & 1
\end{array}\right) \quad \text { for } x \in \mathcal{A} .
$$

We likewise define $e_{21}: \mathcal{A} \rightarrow \widetilde{\mathrm{SL}}_{2}(\mathcal{A})$. For $a \in \mathcal{A}^{\times}$, we define $\widetilde{\Delta}(a) \in \widetilde{\mathrm{SL}}_{2}(\mathcal{A})$ by

$$
\widetilde{\Delta}(a):=e_{21}\left(a^{-1}-1\right) e_{12}(1) e_{21}(a-1) e_{12}\left(-a^{-1}\right)
$$

and observe that $\widetilde{\Delta}(\mathbf{1})=1$. In view of (1), we have $q \circ \widetilde{\Delta}=\Delta$ on $\mathcal{A}^{\times}$.

This means that the restriction $\widetilde{\Delta}: \mathcal{A}_{0}^{\times} \rightarrow \widetilde{\mathrm{SL}}_{2}(\mathcal{A})$ to the identity component $\mathcal{A}_{0}^{\times}$ is the unique continuous lift of the homomorphism $\Delta: \mathcal{A}_{0}^{\times} \rightarrow \mathrm{SL}_{2}(\mathcal{A})_{0}$ to the simply connected covering group $\widetilde{\mathrm{SL}}_{2}(\mathcal{A})$, hence in particular a morphism of Lie groups. This in turn implies that

$$
\widetilde{\Delta}\left(\mathrm{e}^{a}\right)=\exp _{\widetilde{\mathrm{SL}}_{2}(\mathcal{A})}(a h) \text { for } a \in \mathcal{A}
$$

where $\mathrm{e}^{x}=\sum_{n=0}^{\infty} \frac{x^{n}}{n !}$ is the exponential function of the Lie group $\mathcal{A}^{\times}$and $\exp \widetilde{\mathrm{SL}}_{2}(\mathcal{A})$ is the exponential function of the Lie group $\widetilde{\mathrm{SL}}_{2}(\mathcal{A})$.

\subsection{Inducible functionals on $\mathfrak{s l}_{2}(\mathcal{A})$}

Let $(\pi, \mathcal{H})$ be a bounded irreducible $*$-representation of $\mathfrak{s l}_{2}(\mathcal{A})$. We consider the closed subspace

$$
\mathcal{E}:=\mathcal{H}^{\mathfrak{g}^{-}}=\mathcal{H}^{\mathcal{A} e}=\operatorname{ker}(\pi(\mathcal{A} e))
$$


and recall from Proposition 2.2 that the representation $(\rho, \mathcal{E})$ of $\mathfrak{g}^{0}=\mathcal{A} h$ is onedimensional, hence can be written as $\rho(a h)=\lambda(a) 1$ for some $\lambda \in \mathcal{A}^{\prime}$. In this subsection, we shall determine which linear functionals arise from this construction.

To simplify notation, we now put

$$
G:=\widetilde{\mathrm{SL}}_{2}(\mathcal{A}) \text { and } G^{0}:=Z_{G}(h)
$$

In order to determine which $\lambda \in \mathcal{A}^{\prime}$ have the above property, we need to go to the level of Lie groups, that is, we need the holomorphic $*$-representation $\pi_{G}: \widetilde{\mathrm{SL}}_{2}(\mathcal{A}) \rightarrow \mathrm{GL}(\mathcal{H})$ with $\mathrm{d} \pi_{G}=\pi$ (cf. Remark 2.14).

Let $P \in B(\mathcal{H})$ denote the orthogonal projection to $\mathcal{E}$. Then

$$
\phi: G \rightarrow B(\mathcal{E}), \quad \phi(g):=P \pi_{G}(g) P
$$

is a holomorphic function. Observe that the homomorphism $\widetilde{\Delta}: \mathcal{A}_{0}^{\times} \rightarrow G$ defines an isomorphism $\widetilde{\Delta}: \mathcal{A}_{0}^{\times} \rightarrow\left(G^{0}\right)_{0}$ of locally exponential complex Lie groups.

Lemma 3.1. With $G, \phi$ and $\rho$ as above, we have:

(i) $\phi\left(g_{-} g g_{+}\right)=\phi(g)$ for $g \in G, g_{+} \in e_{12}(\mathcal{A}), g_{-} \in e_{21}(\mathcal{A})$.

(ii) $\rho_{G}=\left.\phi\right|_{G^{0}}: G^{0} \rightarrow \mathrm{GL}(\mathcal{E})$ is a representation with $\mathrm{d} \rho_{G}=\rho$.

(iii) $\phi\left(\widetilde{\Delta}\left(\mathrm{e}^{X}\right)\right)=\rho_{G}(\exp (x h))$ for $x \in \mathcal{A}$.

(iv) The group homomorphism $\rho_{G} \circ \tilde{\Delta}: \mathcal{A}_{0}^{\times} \rightarrow \mathrm{GL}(\mathcal{E})$ extends to a polynomial function $F: \mathcal{A} \rightarrow B(\mathcal{E})$.

Proof. We prove the above point by point.

(i) By definition, the elements of $\mathcal{E}$ are fixed by $e_{12}(\mathcal{A})$, so that $\phi\left(g g_{+}\right)=\phi(g)$ for $g \in G$ and $g_{+} \in e_{12}(\mathcal{A})$. From $\phi(g)^{*}=\phi\left(g^{*}\right)$ and $e_{12}(\mathcal{A})^{*}=e_{21}(\mathcal{A})$, we now have $(\mathrm{i})$.

(ii) As $\pi_{G}\left(G^{0}\right)$ normalizes $\mathfrak{g}^{-}$, it preserves $\mathcal{E}$, and this proves (ii).

(iii) follows from (ii) and (3).

(iv) From (i) and the definition of $\widetilde{\Delta}$, we derive

$$
\phi(\widetilde{\Delta}(a))=\phi\left(e_{12}(1) e_{21}(a-1)\right)=P \pi_{G}\left(e_{12}(1) e_{21}(a-1)\right) P=P \mathrm{e}^{\pi(e)} \mathrm{e}^{\pi((a-1) f)} P .
$$

That this function is polynomial in $a$ follows from the existence of a natural number $N \in \mathbb{N}$ with $\pi(\mathcal{A} f)^{N}=\{0\}$ (Proposition 2.2(iii)).

Proposition 3.2. For $\mathfrak{s l}_{2}(\mathcal{A})$, any inducible functional $\lambda: \mathcal{A} \rightarrow \mathbb{C}$ is a finite sum of characters. Any finite sum of involutive characters is inducible. 
Proof. Since $F$ is a polynomial map to $B(\mathcal{E}) \cong \mathbb{C}$ and $F(a b)=F(a) F(b)$ holds for $a, b \in$ $\mathcal{A}_{0}^{\times}=\mathrm{e}^{\mathcal{A}}$, analytic continuation implies that $F(a b)=F(a) F(b)$ for $a, b \in \mathcal{A}$. On the other hand, $F\left(\mathrm{e}^{X}\right)=\mathrm{e}^{\lambda(x)}$ for $x \in \mathcal{A}$. Now Corollary 1.9 implies the existence of $\chi_{1}, \ldots, \chi_{N} \in \Gamma_{\mathcal{A}}$ with $F=\prod_{j=1}^{N} \chi_{j}$. Differentiating in 1 , we obtain

$$
\lambda=\chi_{1}+\cdots+\chi_{N}
$$

For the converse, we only have to show that any involutive character $\chi$ is inducible (cf. Lemma 2.7). This follows from the fact that ev $:_{\chi} \mathfrak{s l}_{2}(\mathcal{A}) \rightarrow \mathfrak{s l}_{2}(\mathbb{C}) \subseteq \mathfrak{g l}_{2}(\mathbb{C})$ is a twodimensional $*$-representation with highest weight $\lambda=\chi$.

Remark 3.3. Every inducible character is involutive. Indeed, suppose that $\chi: \mathcal{A} \rightarrow \mathbb{C}$ is a non-involutive character. The pair $\left(\chi, \chi^{*}\right)$ then defines a surjective homomorphism

$$
\text { ev: } \mathfrak{s l}_{2}(\mathcal{A}) \rightarrow \mathfrak{s l}_{2}(\mathbb{D}) \cong \mathfrak{s l}_{2}(\mathbb{C}) \oplus \mathfrak{s l}_{2}(\mathbb{C})
$$

where the involution on $\mathbb{D}=\mathbb{C}^{2}$ is given by $\left(z_{1}, z_{2}\right)^{*}=\left(\bar{z}_{2}, \bar{z}_{1}\right)$ (cf. Remark 1.7 and Lemma 2.10). Then $\mathfrak{s l}_{2}(\mathbb{D}) \cong \mathfrak{s l}_{2}(\mathbb{C})^{2}$, but the corresponding real form is

$$
\mathfrak{s u}_{2}(\mathbb{D})=\mathfrak{s u}_{2}(\mathbb{C}) \otimes_{\mathbb{R}} \mathbb{D}_{\mathbb{R}}=\left(\mathfrak{s u}_{2}(\mathbb{C}) \otimes(1,1)\right) \oplus\left(\mathfrak{s u}_{2}(\mathbb{C}) \otimes(i,-i)\right)=\left\{\left(z,-z^{*}\right): z \in \mathfrak{s l}_{2}(\mathbb{C})\right\}
$$

As a real Lie algebra, we thus obtain $\mathfrak{s u}_{2}(\mathbb{D}) \cong \mathfrak{s l}_{2}(\mathbb{C})$, which is a simple real Lie algebra with no non-zero bounded unitary representation. This means that $\chi$ is not inducible.

The following theorem closes the gap in the characterization of inducible functionals in Proposition 3.2.

Theorem 3.4. For $\mathfrak{s l}_{2}(\mathcal{A})$, any inducible functional $\lambda: \mathcal{A} \rightarrow \mathbb{C}$ is a finite sum of involutive algebra characters. Conversely, any such sum is inducible.

Proof. In view of Proposition 3.2, it only remains to show that, if a functional $\lambda$ is inducible and a finite sum of characters, then it can be written as a finite sum of involutive characters.

Let $\left(\pi_{\lambda}, \mathcal{H}_{\lambda}\right)$ be the irreducible $*$-representation of $\mathfrak{s l}_{2}(\mathcal{A})$ with highest weight $\lambda$ and unit highest weight vector $v_{\lambda}$ spanning $\mathcal{E}_{\lambda}$. According to Proposition 3.2, we then have

$$
\lambda=\chi_{1}+\cdots+\chi_{N} \quad \text { with } \chi_{j} \in \Gamma_{\mathcal{A}}
$$

We rewrite

$$
\lambda=m_{1} \chi_{1}+\cdots+m_{k} \chi_{k}, \quad \chi_{j} \in \Gamma_{\mathcal{A}}, m_{j} \in \mathbb{N}_{0}
$$


where the $\chi_{j}$ are pairwise different, hence linearly independent in the dual space $\mathcal{A}^{\prime}$. Since $\lambda=\lambda^{*}$, the relation

$$
m_{1} \chi_{1}+\cdots+m_{k} \chi_{k}=m_{1} \chi_{1}^{*}+\cdots+m_{k} \chi_{k}^{*}
$$

implies the existence of an involution $\sigma \in S_{k}$ with $\chi_{j}^{*}=\chi_{\sigma(j)}$ for $j=1, \ldots, k$. If $\sigma(j)=j$, then $\chi_{j}$ is involutive; if $\sigma(j) \neq j$, then $\chi_{j}^{*}=\chi_{\sigma(j)}$. It follows in particular that $m_{j}=m_{\sigma(j)}$. We may thus write

$$
\lambda=\sum_{j=1}^{\ell} a_{j} \eta_{j}+\sum_{j=1}^{n} b_{j}\left(\gamma_{j}+\gamma_{j}^{*}\right), \quad a_{j}, b_{j} \in \mathbb{N}_{0},
$$

where the $\eta_{j}$ are involutive and $\gamma_{j} \neq \gamma_{j}^{*}$. This shows in particular that the ideal

$$
\mathcal{I}:=\bigcap_{j=1}^{N} \operatorname{ker} \chi_{j}=\bigcap_{i} \operatorname{ker} \eta_{i} \cap \bigcap_{j}\left(\operatorname{ker} \gamma_{j} \cap \operatorname{ker} \gamma_{j}^{*}\right)
$$

is $*$-invariant. Since $e \otimes \mathcal{I} \subseteq \mathfrak{g}^{-}$, the Lie algebra $e \otimes \mathcal{I}+h \otimes \mathcal{I}$ annihilates $v_{\lambda}$. This implies that the linear functional $\alpha(X):=\left\langle\pi_{\lambda}(X) v_{\lambda}, v_{\lambda}\right\rangle$ satisfies $\mathfrak{s l}_{2}(\mathcal{I}) \subseteq \operatorname{ker} \alpha$. Next we observe that, for $a \in \mathcal{I}$,

$$
\left\|\pi_{\lambda}(f \otimes a) v_{\lambda}\right\|^{2}=\left\langle\pi_{\lambda}(f \otimes a)^{*} \pi_{\lambda}(f \otimes a) v_{\lambda}, v_{\lambda}\right\rangle=\left\langle\pi_{\lambda}\left(\left[(f \otimes a)^{*}, f \otimes a\right]\right) v_{\lambda}, v_{\lambda}\right\rangle \in \alpha\left(\mathfrak{s} \mathfrak{l}_{2}(\mathcal{I})\right)=\{0\}
$$

whence $v_{\lambda} \in \mathcal{H}_{\lambda}^{\mathfrak{s l}_{2}(\mathcal{I})}$. Since $\mathfrak{s l}_{2}(\mathcal{I}) \unlhd \mathfrak{s l}_{2}(\mathcal{A})$ is an ideal, the subspace $\mathcal{H}_{\lambda}^{\mathfrak{s l}_{2}(\mathcal{I})}$ is invariant under $\mathfrak{s l}_{2}(\mathcal{A})$. As it contains the cyclic vector $v_{\lambda}$, it follows that $\mathfrak{s l}_{2}(\mathcal{I}) \subseteq \operatorname{ker} \pi_{\lambda}$ (cf. [30, Lemma IX.1.3] for similar arguments). Therefore, the representation $\pi_{\lambda}$ factors through a representation $\bar{\pi}_{\lambda}$ of the involutive quotient algebra $\mathfrak{s l}_{2}(\mathcal{A} / \mathcal{I})$. We now have to understand the structure of this algebra.

With (4) and Lemma 2.10, we see that codim $\mathcal{I}=\ell+2 n$. Accordingly, $\mathcal{A} / \mathcal{I}$ is isomorphic to the algebra $\mathbb{C}^{\ell} \oplus \mathbb{D}^{n}$ with the involution

$$
\left(x_{1}, \ldots, x_{\ell}, y_{1}, \ldots, y_{n}, z_{1}, \ldots, z_{n}\right)^{*}=\left(\bar{x}_{1}, \ldots, \bar{x}_{\ell}, \bar{z}_{1}, \ldots, \bar{z}_{n}, \bar{y}_{1}, \ldots, \bar{y}_{n}\right) .
$$

We thus obtain

$$
\mathfrak{s u}_{2}(\mathcal{A} / \mathcal{I}) \cong \mathfrak{s u}_{2}(\mathbb{C})^{\ell} \oplus \mathfrak{s l}_{2}(\mathbb{C})^{n}
$$

(cf. Remark 3.3). Since $\mathfrak{s l}_{2}(\mathbb{C})$ has no non-zero bounded $*$-representations, $\bar{\pi}_{\lambda}$ is trivial on the corresponding factors, and this in turn implies that $n=0$. 


\section{Lie Algebras of Smooth Sections}

Building on Corollary 2.12, we now extend our classification results to Lie algebras of smooth sections of Lie algebra bundles $\mathfrak{K} \rightarrow X$, where the typical fiber $\mathfrak{k}$ of $\mathfrak{K}$ is compact semisimple, and $X$ is a $\sigma$-compact smooth manifold with compact boundary $\partial X$. This includes in particular the Fréchet-Lie algebras $\mathfrak{g a u}(P)$ of infinitesimal gauge transformations of principal bundles $P \rightarrow X$ with compact semisimple structure group $K$.

\subsection{Lie algebra bundles}

Let $q: \mathfrak{K} \rightarrow X$ be a smooth Lie algebra bundle over $X$ whose typical fiber $\mathfrak{k}$ is a finitedimensional Lie algebra. Let $\Gamma(\mathfrak{K})$ denote the space of sections $s: X \rightarrow \mathfrak{K}$ that are smooth on the interior $X^{\circ}$, and whose derivatives extend continuously to the boundary. We endow $\Gamma(\mathfrak{K})$ with the smooth compact open topology obtained from the embedding

$$
\Gamma(\mathfrak{K}) \hookrightarrow \prod_{n \in \mathbb{N}_{0}} C\left(T^{n} X, T^{n} \mathfrak{K}\right), \quad s \mapsto\left(T^{n} s\right)_{n \in \mathbb{N}_{0}},
$$

and the compact open topology on the spaces $C\left(T^{n} X, T^{n} \mathfrak{K}\right)$. This turns $\Gamma(\mathfrak{K})$ into a Fréchet-Lie algebra with respect to the pointwise bracket

$$
\left[s_{1}, s_{2}\right](X):=\left[s_{1}(X), s_{2}(X)\right]
$$

(cf. [31, Theorem II.2.7]).

We write $X=\bigcup_{n \in \mathbb{N}} X_{n}$, where $X_{n}$ is a compact submanifold with boundary, $X=$ $\bigcup_{n} X_{n}$ and $X_{n} \subseteq X_{n+1}^{0}$ for $n \in \mathbb{N}$. (This is possible because we required $\partial X$ to be compact.) We also put $\mathfrak{K}_{n}:=\mathfrak{K}_{X_{n}}$ and observe that the restriction map $r_{n}: \Gamma(\mathfrak{K}) \rightarrow \Gamma\left(\mathfrak{K}_{n}\right)$ is surjective for every $n$ (cf. [55]). Therefore, the embedding

$$
\Gamma(\mathfrak{K}) \rightarrow \underset{\longleftarrow}{\lim } \Gamma\left(\mathfrak{K}_{n}\right)
$$

is a continuous bijective linear map between Fréchet spaces, hence a topological isomorphism by the Open Mapping Theorem (cf. [41]).

The space $\Gamma_{c}(\mathfrak{K})$ of compactly supported smooth functions is the union of the closed ideals

$$
\Gamma(\mathfrak{K})_{X_{n}}:=\left\{s \in \Gamma(\mathfrak{K}): \operatorname{supp}(s) \subseteq X_{n}\right\} \unlhd \Gamma(\mathfrak{K})
$$

which are Fréchet spaces. We endow $\Gamma_{C}(\mathfrak{K}) \cong \lim _{\longrightarrow} \Gamma(\mathfrak{K})_{X_{n}}$ with the corresponding locally convex direct limit topology which turns it into an LF-Lie algebra, that is, an LF-space with a continuous Lie bracket [19, Corollary F.24, Remark F.28]. 
The following proposition reduces the problem of describing the bounded unitary representations of $\Gamma(\mathfrak{K})$ to the case where $X$ is a compact manifold with boundary.

Proposition 4.1. For every bounded unitary representation $(\pi, \mathcal{H})$ of the Fréchet-Lie algebra $\Gamma(\mathfrak{K})$, there exists a compact submanifold $Y \subseteq X$ with boundary and a bounded unitary representation $(\bar{\pi}, \mathcal{H})$ of $\Gamma\left(\left.\mathfrak{K}\right|_{Y}\right)$ such that $\pi(s)=\bar{\pi}\left(\left.s\right|_{Y}\right)$ for every $s \in \Gamma(\mathfrak{K})$.

Proof. Since $\Gamma(\mathfrak{K})$ is the projective limit of the Fréchet spaces $\Gamma\left(\mathfrak{K}_{n}\right)$, there exists an $n \in \mathbb{N}$ and a continuous seminorm $p$ on $\Gamma\left(\mathfrak{K}_{n}\right)$ such that

$$
\|\pi(s)\| \leq p\left(\left.s\right|_{X_{n}}\right) \quad \text { for } s \in \Gamma(\mathfrak{K}) .
$$

This implies that $\pi$ vanishes on the kernel of the restriction map $r_{n}$, and since $r_{n}$ is a quotient map, the assertion follows with $Y=X_{n}$.

Remark 4.2. Suppose that $\mathfrak{k}$ is compact. Let $\mathfrak{k}=\mathfrak{z}(\mathfrak{k}) \oplus[\mathfrak{k}, \mathfrak{k}]$ denote the decomposition of $\mathfrak{k}$ into center and the semisimple commutator algebra. Since this decomposition is invariant under the full automorphism group Aut(k), it induces a direct sum decomposition $\mathfrak{K} \cong Z(\mathfrak{K}) \oplus[\mathfrak{K}, \mathfrak{K}]$ of Lie algebra bundles, which in turn leads to

$$
\Gamma(\mathfrak{K}) \cong \Gamma(Z(\mathfrak{K})) \oplus \Gamma([\mathfrak{K}, \mathfrak{K}]) .
$$

If $(\pi, \mathcal{H})$ is a bounded factor representation of $\Gamma(\mathfrak{K})$, then $\pi(\Gamma(Z(\mathfrak{K}))) \subseteq$ $Z\left(\pi(\Gamma(\mathfrak{K}))^{\prime \prime}\right)=\mathbb{C} 1$. Therefore, the representation $\pi$ is a tensor product of a onedimensional unitary representation of the abelian Lie algebra $\Gamma(Z(\mathfrak{K}))$ and a factor representation of $\Gamma([\mathfrak{K}, \mathfrak{K}])$. Since every continuous linear map $\lambda: \Gamma(Z(\mathfrak{K})) \rightarrow i \mathbb{R}$ defines a one-dimensional unitary representation, the classification of bounded factor representations of $\Gamma(\mathfrak{K})$ reduces to the corresponding problem for $\Gamma([\mathfrak{K}, \mathfrak{K}])$.

Example 4.3. (a) Typically, Lie algebra bundles arise as $\mathfrak{K}:=\operatorname{Ad}(P)$ for a smooth $K$-principal bundle $q: P \rightarrow X$, where $K$ is a Lie group with Lie algebra $\mathfrak{k}$. The adjoint bundle $\operatorname{Ad}(P):=P \times_{\mathrm{Ad}} \mathfrak{k} \rightarrow X$ is the orbit space of the action $K \curvearrowright P \times \mathfrak{k}$ defined by $k \cdot(p, x):=$ $\left(p k^{-1}, \operatorname{Ad}(k) X\right)$.

The group of vertical bundle automorphisms of $P$ is called the gauge group $\operatorname{Gau}(P)$. It is a locally convex Lie group if $M$ is compact. Each gauge transformation $g \in \mathrm{Gau}(P)$ is of the form $g(p)=p \widetilde{g}(p)$ with $\widetilde{g} \in C^{\infty}(P, K)^{K}$, that is, $\widetilde{g}(p k)=k^{-1} \widetilde{g}(p) k$ for all $p \in P, k \in K$. The map $g \mapsto \widetilde{g}$ is an isomorphism of locally exponential Lie groups 
$\operatorname{Gau}(P) \rightarrow C^{\infty}(P, K)^{K}$. Accordingly, we obtain an isomorphism of Lie algebras

$$
\mathfrak{g a u}(P)=\Gamma(\operatorname{Ad}(P)) \rightarrow C^{\infty}(P, \mathfrak{k})^{K}:=\left\{f \in C^{\infty}(P, \mathfrak{k}):(\forall p \in P, k \in K) f(p k)=\operatorname{Ad}(k)^{-1} f(p)\right\}
$$

(b) If $\rho: K \rightarrow \mathrm{U}(V)$ is a continuous finite-dimensional unitary representation of $K$, then we obtain an associated vector bundle $\mathbb{V}:=P \times_{\rho} V$ as the orbit space of the action $K \curvearrowright P \times V$ defined by $k \cdot(p, v):=\left(p k^{-1}, \rho(k) v\right)$. As in the case of the adjoint bundle, one identifies sections $s \in \Gamma(\mathbb{V})$ with equivariant functions $\widetilde{s} \in C^{\infty}(P, V)^{K}$.

The gauge group $\operatorname{Gau}(P)$ acts on $\mathbb{V}$ by bundle automorphisms via

$$
g \cdot[p, v]:=[g(p), v]=[p \widetilde{g}(p), v]=[p, \rho(\widetilde{g}(p)) v]
$$

For any Radon measure $\mu$ on $X$, we obtain on the space $\Gamma(\mathbb{V})$ of smooth sections of $\mathbb{V}$ a scalar product by

$$
\langle s, t\rangle:=\int_{X}\langle s(x), t(x)\rangle \mathrm{d} \mu(x) .
$$

On the Hilbert completion $\Gamma^{2}(\mathbb{V}, \mu)$ of $\Gamma(\mathbb{V})$, this yields a unitary representation of $\operatorname{Gau}(P)$ by $(g \cdot s)(x):=g \cdot s(x)$. If $s$ is identified with $\widetilde{s} \in C^{\infty}(P, V)^{K}$, this reads $(g \cdot \widetilde{s})(p)=$ $\rho(\widetilde{g}(p)) \widetilde{S}(p)$. If $X$ is compact, then this representation $\mathrm{Gau}(P) \rightarrow \mathrm{U}\left(\Gamma^{2}(\mathbb{V}, \mu)\right)$ is norm continuous, and the corresponding derived representation

$$
(\xi . \widetilde{s})(p)=\mathrm{d} \rho(\xi(p)) \widetilde{s}(p)
$$

is a bounded unitary representation of $\mathfrak{g a \mathfrak { u }}(P)$.

Since the commutant of this representation always contains the multiplications with elements of $L^{\infty}(X, \mu)$, it is irreducible if and only if $V$ is irreducible, and $\mu$ is non-zero and supported in a single point $x_{0} \in X$. Fix $p_{0} \in P$ with $q\left(p_{0}\right)=x_{0}$. Then $\Gamma^{2}(\mathbb{V}, \mu) \rightarrow V, s \mapsto \widetilde{s}\left(p_{0}\right)$ is a unitary equivalence intertwining the representation of $\operatorname{Gau}(P)$ on $\Gamma^{2}(\mathbb{V}, \mu)$ with the evaluation representation on $V$ by $\pi_{p_{0}}(g):=\rho\left(\widetilde{g}\left(p_{0}\right)\right)$.

\subsection{Local structure of irreducible bounded representations}

We proceed with the classification of irreducible bounded unitary representations in terms of evaluation representations. We aim to prove that they are tensor products of evaluation representations. In order to do this, we investigate the local structure of bounded unitary representations. 
Definition 4.4. Let $x \in X$ and let $(\rho, V)$ be a bounded representation of $\mathfrak{K}_{X} \cong \mathfrak{k}$. Then $\pi(s):=\rho(s(x))$ defines a bounded unitary representation of $\Gamma(\mathfrak{K})$. We call these representations evaluation representations. Note that $\pi$ is irreducible if and only if $\rho$ is.

Remark 4.5. In view of the paracompactness of $X$, there exists a locally finite open covering $\left(U_{j}\right)_{j \in J}$ by relatively compact subsets $U_{j} \subseteq X$ for which $\mathfrak{K}$ is trivial on an open neighborhood of $\bar{U}_{j}$. Then the space $\Gamma_{C}\left(\left.\mathfrak{K}\right|_{U_{j}}\right)$ of sections of $\mathfrak{K}$ with support contained in $U_{j}$ is isomorphic to $C_{c}^{\infty}\left(U_{j}, \mathfrak{k}\right)$ and a partition of unitary argument shows that

$$
\Gamma_{C}(\mathfrak{K})=\sum_{j \in J} \Gamma_{C}\left(\left.\mathfrak{K}\right|_{U_{j}}\right),
$$

where the $\Gamma_{C}\left(\left.\mathfrak{K}\right|_{U_{j}}\right)$ are ideals isomorphic to $C_{C}^{\infty}\left(U_{j}, \mathfrak{k}\right)$.

The following lemma can also be found as Lemma 2.10 in Eyni's recent preprint [13].

Lemma 4.6. If $\mathfrak{k}$ is perfect, that is, $\mathfrak{k}=[\mathfrak{k}, \mathfrak{k}]$, then also $\Gamma_{C}(\mathfrak{K})$ is perfect.

Proof. Since $\Gamma_{C}(\mathfrak{K})$ is a sum of subalgebras of the form $C_{C}^{\infty}(X, \mathfrak{k})$ (Remark 4.5), it suffices to show that $C_{C}^{\infty}(X, \mathfrak{k}) \simeq C_{C}^{\infty}(X, \mathbb{R}) \otimes_{\mathbb{R}} \mathfrak{k}$ is perfect for every smooth manifold $X$. Since $\mathfrak{k}$ is perfect, every $x \in \mathfrak{k}$ can be written as $x=\sum_{j=1}^{k}\left[y_{j}, z_{j}\right]$ with $y_{j}, z_{j} \in \mathfrak{k}$. For $f \in C_{c}^{\infty}(X, \mathbb{R})$, we choose a function $\chi \in C_{c}^{\infty}(X, \mathbb{R})$ with $\left.\chi\right|_{\operatorname{supp}(f)}=1$. Then

$$
\sum_{j=1}^{k}\left[y_{j} \otimes \chi, z_{j} \otimes f\right]=\sum_{j=1}^{k}\left[y_{j}, z_{j}\right] \otimes \chi f=x \otimes f
$$

shows that $C_{C}^{\infty}(X, \mathfrak{k})$ is perfect.

Note that the above lemma applies in particular to compact semisimple Lie algebras $\mathfrak{k}$, which are automatically perfect.

Lemma 4.7. Let $(\rho, \mathcal{H})$ be a finite tensor product of irreducible evaluation representations at different points for an ideal $\Gamma_{C}\left(\left.\mathfrak{K}\right|_{U}\right)\left(U \subseteq X\right.$ open) of $\Gamma_{C}(\mathfrak{K})$. Then $(\rho, \mathcal{H})$ extends uniquely to a bounded unitary representation $(\bar{\rho}, \mathcal{H})$ of $\Gamma_{c}(\mathfrak{K})$ on the same space. It is again a finite tensor product of irreducible evaluation representations at different points.

Proof. Since $\rho$ is a finite tensor product of evaluation representations, the existence of the extension follows from the obvious extensions of evaluation representations. 
To see that the extension is unique, note that a finite tensor product of irreducible evaluation representations is itself irreducible, provided that one evaluates at different points $x$ of $X$. Now suppose that $\tilde{\rho}$ and $\bar{\rho}$ are two extensions of $\rho$. Then, for each $x \in \Gamma_{C}(\mathfrak{K})$, the operator $\widetilde{\rho}(x)-\bar{\rho}(x)$ commutes with $\rho\left(\Gamma_{C}\left(\left.\mathfrak{K}\right|_{U_{j}}\right)\right)$, so that Schur's Lemma implies that it is of the form $\alpha(x) 1$ for some $\alpha(x) \in i \mathbb{R}$. Then $\alpha: \Gamma_{C}(\mathfrak{K}) \rightarrow \mathbb{R}$ is a one-dimensional representation, hence vanishes on all brackets. As $\Gamma_{C}(\mathfrak{K})$ is perfect by Lemma 4.6, $\alpha=0$, and therefore $\tilde{\rho}=\bar{\rho}$.

Lemma 4.8. Let $\mathfrak{g}$ be a Lie algebra and $\mathfrak{n} \unlhd \mathfrak{g}$ be an ideal. Suppose that $\pi: \mathfrak{g} \rightarrow \mathfrak{u}(\mathcal{H})$ is a unitary factor representation. Then

(i) $\left.\pi\right|_{\mathfrak{n}}$ is a factor representation.

(ii) If $\left.\pi\right|_{\mathfrak{n}}$ is a type I representation, that is, a multiple of an irreducible representation $(\rho, V)$, and if $\rho$ extends to a bounded irreducible representation $\left(\pi_{2}, \mathcal{H}\right)$ of $\mathfrak{g}$, then there exists a bounded representation $\left(\pi_{1}, \mathcal{H}_{1}\right)$ of $\mathfrak{g}$ such that $\pi \cong \pi_{1} \otimes \pi_{2}$ and $\mathfrak{n} \subseteq \operatorname{ker} \pi_{1}$. Then $\pi_{1}$ is a factor representation which is irreducible if and only if $\pi$ is irreducible.

Proof. (i) Let $\mathcal{M}:=\pi(\mathfrak{n})^{\prime \prime} \subseteq B(\mathcal{H})$ denote the bicommutant of $\pi(\mathfrak{n})$. The fact that $\mathfrak{n} \unlhd \mathfrak{g}$ is an ideal implies that $\mathcal{M}$ is invariant under $\operatorname{ad}(\pi(\mathfrak{g}))$, so that we obtain for each $x \in \mathfrak{g}$ a derivation $\operatorname{ad}(\pi(x))$ of $\mathcal{M}$. Since every derivation of a von Neumann algebra is inner [46, Theorem XI.3.5], $\operatorname{ad}(\pi(\mathfrak{g}))$ annihilates the center of $\mathcal{M}$, so that $Z(\mathcal{M}) \subseteq \pi(\mathfrak{g})^{\prime}$. On the other hand, $Z(\mathcal{M}) \subseteq \mathcal{M} \subseteq \pi(\mathfrak{g})^{\prime \prime}$, so that $Z(\mathcal{M}) \subseteq Z\left(\pi(\mathfrak{g})^{\prime \prime}\right)=\mathbb{C} 1$ since $\pi(\mathfrak{g})^{\prime \prime}$ is a factor. We conclude that $\mathcal{M}$ is also a factor.

(ii) If $\mathcal{M}$ is of type I, there exists an irreducible bounded unitary representation $\left(\rho, \mathcal{H}_{2}\right)$ of $\mathfrak{n}$ and a Hilbert space $\mathcal{H}_{1}$ (the multiplicity space) such that $\mathcal{H} \cong \mathcal{H}_{1} \hat{\otimes} \mathcal{H}_{2}$ and $\left.\pi\right|_{\mathfrak{n}}=\mathbf{1}_{\mathcal{H}_{1}} \otimes \rho$. Extending the irreducible representation $\rho$ to a representation $\pi_{2}$ of $\mathfrak{g}$ on $\mathcal{H}_{2}$, we obtain the representation $\tilde{\pi}_{2}:=1 \otimes \pi_{2}$ of $\mathfrak{g}$ on $\mathcal{H}=\mathcal{H}_{1} \hat{\otimes} \mathcal{H}_{2}$ which coincides on $\mathfrak{n}$ with $\pi$. For each $x \in \mathfrak{g}$, the operator

$$
\tilde{\pi}_{1}(x):=\pi(x)-\tilde{\pi}_{2}(x)
$$

commutes with $\pi(\mathfrak{n})$ with generates the von Neumann algebra $\mathcal{M} \cong 1 \otimes B\left(\mathcal{H}_{2}\right)$. Therefore,

$$
\tilde{\pi}_{1}(x)=\pi_{1}(x) \otimes 1 \in B\left(\mathcal{H}_{1}\right) \otimes \mathbf{1}_{\mathcal{H}_{2}} \quad \text { for some } \pi_{1}(x) \in B\left(\mathcal{H}_{1}\right),
$$

which implies in particular that $\tilde{\pi}_{1}(x)$ commutes with $\tilde{\pi}_{2}(\mathfrak{g})$. This leads to

$$
\pi(x)=\pi_{1}(x) \otimes 1+1 \otimes \pi_{2}(x),
$$


that is, $\pi \cong \pi_{1} \otimes \pi_{2}$. Now

$$
\pi(\mathfrak{g})^{\prime} \subseteq \pi(\mathfrak{n})^{\prime}=(1 \otimes \mathcal{M})^{\prime}=B\left(\mathcal{H}_{1}\right) \otimes 1
$$

leads to

$$
\pi(\mathfrak{g})^{\prime}=\pi(\mathfrak{g})^{\prime} \cap\left(B\left(\mathcal{H}_{1}\right) \otimes 1\right)=\pi_{1}(\mathfrak{g})^{\prime} \otimes 1 \cong \pi_{1}(\mathfrak{g})^{\prime},
$$

and further to

$$
\pi(\mathfrak{g})^{\prime \prime}=\pi_{1}(\mathfrak{g})^{\prime \prime} \otimes B\left(\mathcal{H}_{2}\right) .
$$

We conclude that

$$
\mathbb{C} 1=Z\left(\pi(\mathfrak{g})^{\prime \prime}\right)=Z\left(\pi_{1}(\mathfrak{g})^{\prime \prime}\right) \otimes 1,
$$

so that $\pi_{1}$ is a factor representation. We also see with Schur's Lemma that $\pi$ is irreducible if and only if $\pi_{1}$ is irreducible.

Lemma 4.9. Suppose that $\mathfrak{k}$ is compact semisimple. Let $(\pi, \mathcal{H})$ be a bounded unitary factor (irreducible) representation of $\Gamma_{C}(\mathfrak{K})$ and $U \subseteq X$ an open relatively compact subset for which the bundle $\mathfrak{K}$ is trivial on an open neighborhood $V$ of $\bar{U}$. Then the following assertions hold:

(i) The restriction of $\pi$ to the ideal $\mathfrak{n}:=\Gamma_{C}\left(\left.\mathfrak{K}\right|_{U}\right) \cong C_{C}^{\infty}(U, \mathfrak{k})$ extends to a representation of the topological Lie algebra $C_{C}^{\infty}(U, \mathfrak{k}) \rtimes \mathfrak{k} \cong \mathfrak{k} \otimes_{\mathbb{R}} C_{C}^{\infty}(U)_{+}$.

(ii) There exists a bounded factor (irreducible) representation $\left(\pi_{1}, \mathcal{H}_{1}\right)$ of $\Gamma_{C}(\mathfrak{K})$ and a finite tensor product $\left(\pi_{2}, \mathcal{H}_{2}\right)$ of irreducible evaluation representations at different points such that $\pi \cong \pi_{1} \otimes \pi_{2}$ and $\mathfrak{n} \subseteq \operatorname{ker} \pi_{1}$.

Proof. Let $\mathcal{M}:=\pi(\mathfrak{n})^{\prime \prime} \subseteq B(\mathcal{H})$ denote the bicommutant of $\pi(\mathfrak{n})$. In view of Lemma 4.8, this is a factor. If $\pi(\mathfrak{n})=\{0\}$, there is nothing to show. We may therefore assume that $\mathfrak{n} \nsubseteq \operatorname{ker} \pi$. Since $\mathfrak{n} \unlhd \mathfrak{g}:=\Gamma_{C}(\mathfrak{K})$ is an ideal, we obtain a homomorphism

$$
\alpha: \mathfrak{g} \rightarrow \operatorname{der}_{*}(\mathcal{M}), \quad \alpha(s)(A):=[\pi(s), A] .
$$

Since every derivation of a von Neumann algebra is inner [46, Theorem XI.3.5], $\operatorname{der}(\mathcal{M}) \cong$ $\mathcal{M} / Z(\mathcal{M})$ as Banach-Lie algebras. This in turn implies that the $*$-derivations of $\mathcal{M}$ are induced by elements in $\mathfrak{u}(\mathcal{M}):=\left\{A \in \mathcal{M}: A^{*}=-A\right\}$, that is,

$$
\operatorname{der}_{*}(\mathcal{M}):=\left\{D \in \operatorname{der}(\mathcal{M}):(\forall M \in \mathcal{M}) D\left(M^{*}\right)=D(M)^{*}\right\} \cong \mathfrak{u}(\mathcal{M}) / Z(\mathfrak{u}(\mathcal{M})) .
$$

Let $h \in C_{C}^{\infty}(V, \mathbb{R})$ be such that $\left.h\right|_{U}=1$. For $x \in \mathfrak{k}$ and the corresponding section

$$
x \otimes h \in \mathfrak{k} \otimes C_{C}^{\infty}(V, \mathbb{R}) \cong C_{c}^{\infty}(V, \mathfrak{k}) \cong \Gamma_{C}\left(\left.\mathfrak{K}\right|_{V}\right) \subseteq \Gamma_{C}(\mathfrak{K})
$$


we then have

$$
\alpha(x \otimes h) \pi(s)=\pi([x, s]), \quad s \in C_{C}(U, \mathfrak{k}) .
$$

In particular, $\alpha(x \otimes h)$ does not depend on the choice of $h$, which leads to a homomorphism

$$
\bar{\alpha}: \mathfrak{k} \rightarrow \operatorname{der}_{*}(\mathcal{M}), \quad x \mapsto \alpha(x \otimes h) .
$$

The pullback along $\bar{\alpha}$ of the central extension

$$
\mathfrak{u}(Z(\mathcal{M})) \rightarrow \mathfrak{u}(\mathcal{M}) \rightarrow \operatorname{der}_{*}(\mathcal{M})
$$

yields a $\mathfrak{u}(Z(\mathcal{M})$ )-valued 2-cocycle on $\mathfrak{k}$, which is trivial because $\mathfrak{k}$ is semisimple. We conclude that there exists a homomorphism

$$
\tilde{\alpha}: \mathfrak{k} \rightarrow \mathfrak{u}(\mathcal{M}) \quad \text { with } \alpha(x)=\operatorname{ad}(\widetilde{\alpha}(x)) \text { for } x \in \mathfrak{k}
$$

Then $\widetilde{\alpha}$ is a bounded unitary representation of $\mathfrak{k}$. Because of (5),

$$
\bar{\pi}: \mathfrak{n}_{+}:=C_{C}(U, \mathfrak{k}) \rtimes \mathfrak{k} \rightarrow \mathfrak{u}(\mathcal{M}) \subseteq \mathfrak{u}(\mathcal{H}), \quad(s, x) \mapsto \pi(s)+\widetilde{\alpha}(x)
$$

defines a bounded unitary representation whose range lies in $\mathfrak{u}(\mathcal{M})$.

Let $\mathcal{A}:=C^{*}\left(\bar{\pi}\left(\mathfrak{n}_{+}\right)\right) \subseteq B(\mathcal{H})$ denote the $C^{*}$-algebra generated by $\bar{\pi}\left(\mathfrak{n}_{+}\right)$. Then every irreducible representation $(\alpha, \mathcal{F})$ of $\mathcal{A}$ defines a bounded irreducible representation of $\mathfrak{n}_{+}$, hence is finite dimensional by Corollary 2.12. In particular, the image of $\mathcal{A}$ in every irreducible representation contains the compact operators, so that $\mathcal{A}$ is type I [42]. We conclude that the factor $\mathcal{M}=\mathcal{A}^{\prime \prime}$ is of type I and that $\bar{\pi}$ is a factor representation of type I, hence a multiple of some irreducible representation $(\bar{\rho}, V)$ whose restriction $(\rho, V)$ to $\mathfrak{n}$ is also irreducible. Now $\rho$ is a finite tensor product of irreducible evaluation representations in different points for $\mathfrak{k} \otimes C_{C}^{\infty}(U, \mathbb{R})_{+}$(cf. Theorem 2.11), and since the restriction to $\mathfrak{n}$ is irreducible, none of the corresponding characters of $C_{c}^{\infty}(U, \mathbb{R})_{+}$vanishes on the ideal $C_{C}^{\infty}(U, \mathbb{R})$. Hence, they are given by evaluations in points of $U$ (Example 1.2). In view of Lemma 4.7, $\rho$ extends uniquely to an irreducible bounded unitary representation $\left(\pi_{2}, \mathcal{H}_{2}\right)$ of $\mathfrak{g}$. Now Lemma 4.8(ii) applies and the assertion follows.

Theorem 4.10. Suppose that $X$ is a smooth manifold with compact boundary and that $\mathfrak{K} \rightarrow X$ is a smooth Lie algebra bundle whose typical fiber $\mathfrak{k}$ is a compact semisimple Lie algebra. Let $C \subseteq X$ be a compact subset and let $(\pi, \mathcal{H})$ be a bounded unitary factor (irreducible) representation of $\Gamma_{c}(\mathfrak{K})$. Then there exists a bounded factor (irreducible) representation $\left(\pi_{1}, \mathcal{H}_{1}\right)$ and a finite tensor product $\left(\pi_{2}, \mathcal{H}_{2}\right)$ of irreducible evaluation 
representations in different points for $\Gamma_{C}(\mathfrak{K})$ such that $\pi \cong \pi_{1} \otimes \pi_{2}$ and $\Gamma(\mathfrak{K})_{C}=$ $\{s \in \Gamma(\mathfrak{K}): \operatorname{supp}(s) \subseteq C\} \subseteq \operatorname{ker} \pi_{1}$.

Proof. Let $\left(U_{j}\right)_{j \in J}$ be as in Remark 4.5. Since $C$ is compact and the covering $\left(U_{j}\right)$ is locally finite, the set $F:=\left\{j \in J: U_{j} \cap C \neq \emptyset\right\}$ is finite and we may w.l.o.g. assume that $F=\{1, \ldots, N\}$. Then $\mathfrak{n}:=\sum_{j \in F} \mathfrak{g}_{j}$ is an ideal of $\mathfrak{g}:=\Gamma_{C}(\mathfrak{K})$, where $\mathfrak{g}_{j}:=\Gamma_{C}\left(\left.\mathfrak{K}\right|_{U_{j}}\right) \cong C_{C}^{\infty}\left(U_{j}, \mathfrak{k}\right)$. From $C \subseteq \bigcup_{j \in F} U_{j}$, it follows that $\operatorname{supp}(s) \subseteq C$ implies $s \in \mathfrak{n}$.

If $\mathfrak{n} \subseteq \operatorname{ker} \pi$, we put $\pi_{1}:=\pi$, and there is nothing to show. If this is not the case, there exists a minimal $j \in F$ for which $\mathfrak{g}_{j} \not \subset \operatorname{ker} \pi$. Then Lemma 4.9 leads to a tensor product decomposition $\pi=\rho_{j} \otimes \rho_{j}^{\prime}$, where $\rho_{j}$ vanishes on $\sum_{i \leq j} \mathfrak{g}_{j}$ and $\left.\rho_{j}^{\prime}\right|_{\mathfrak{g}_{j}}$ is a finite tensor product of irreducible evaluation representations in different points, hence in particular irreducible. If $\mathfrak{n} \nsubseteq \operatorname{ker} \rho_{j}$, we apply the same argument to $\rho_{j}$, where $j^{\prime} \in\{i \in F: i>j\}$

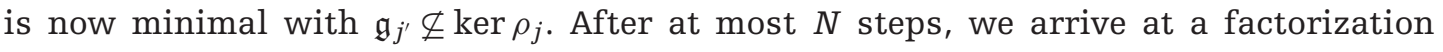
$\pi=\pi_{1} \otimes \pi_{2}$, where $\pi_{1}$ vanishes on $\mathfrak{n}$ and $\pi_{2}$ is a finite tensor product of irreducible evaluation representations. Because $\rho_{j}$ vanishes on $\sum_{i \leq j} \mathfrak{g}_{j}$, the new points that one obtains at each step cannot coincide with points that one already had. This completes the proof.

\subsection{Classification of irreducible bounded representations}

Using the preceding theorem on the representations of $\Gamma_{C}(\mathfrak{K})$, we can now prove our main result on the Fréchet-Lie algebra $\Gamma(\mathfrak{K})$ of all smooth sections. In particular, it shows that all irreducible bounded unitary representations are finite dimensional.

Theorem 4.11. Suppose that $X$ is a smooth manifold with compact boundary and that $\mathfrak{K} \rightarrow X$ is a smooth Lie algebra bundle whose typical fiber $\mathfrak{k}$ is a compact semisimple Lie algebra. Then every bounded irreducible unitary representation $\pi$ of $\Gamma(\mathfrak{K})$ is equivalent to a finite tensor product of irreducible evaluation representations at different points. That is, there exists a finite subset $\mathbf{x} \subseteq X$ and irreducible representations $\rho_{X}$ of $\mathfrak{K}_{X}$ such that $\pi \simeq \pi_{\mathbf{x}, \rho}:=\bigotimes_{X \in \mathbf{X}} \rho_{X} \circ \mathrm{ev}_{X}$. Two such representations $\pi_{\mathbf{X}, \rho}$ and $\pi_{\mathbf{x}^{\prime}, \rho^{\prime}}$ are equivalent if and only if $\mathbf{x}=\mathbf{x}^{\prime}$ and $\rho_{X} \simeq \rho_{X}^{\prime}$ for all $x \in \mathbf{x}$.

Proof. In view of Proposition 4.1, we may w.l.o.g. assume that $X$ is compact. Then $\pi \simeq \pi_{\mathbf{x}, \rho}$ follows from the preceding theorem with $C=X$. Since the evaluation map $\operatorname{ev}_{\mathbf{x} \cup \mathbf{x}^{\prime}}: \Gamma(\mathfrak{K}) \rightarrow \bigoplus_{x \in \mathbf{x} \cup \mathbf{x}^{\prime}} \mathfrak{K}_{X}$ is surjective, $\pi_{\mathbf{x}, \rho} \simeq \pi_{\mathbf{x}^{\prime}, \rho^{\prime}}$ implies $\mathbf{x}=\mathbf{x}^{\prime}$, as well as $\rho_{X} \simeq \rho_{X}^{\prime}$.

Remark 4.12. Let $\mathfrak{g}=\Gamma(\mathfrak{K})$ be as in Theorem 4.11 and let $(\rho, \mathcal{H})$ be a (not necessarily irreducible) bounded unitary representation of $\mathfrak{g}$. We consider the $C^{*}$-algebra 
$\mathcal{A}:=C^{*}(\rho(\mathfrak{g})) \subseteq B(\mathcal{H})$ generated by $\pi(\mathfrak{g})$. For every irreducible representation $(\beta, \mathcal{K})$ of $\mathcal{A}$, we obtain an irreducible bounded representation $(\beta \circ \rho, \mathcal{K})$ of $\mathfrak{g}$. Theorem 4.11 now implies that $\operatorname{dim} \mathcal{K}<\infty$, so that $\beta(\mathcal{A})=B(\mathcal{K})$ consists of compact operators. This means that $\mathcal{A}$ is a CCR (or liminal) $C^{*}$-algebra. As $X$ is $\sigma$-compact and $\mathfrak{k}$ is finite dimensional, it further follows that $\mathfrak{g}$ is separable, and this implies that $\mathcal{A}$ is separable. Now [11, Theorem 8.6.6] implies that the identical representation of $\mathcal{A}$ on $\mathcal{H}$ can be represented in a canonical fashion as a direct integral of irreducible representations over the space $\hat{\mathcal{A}}$ of (equivalence classes of) irreducible representations of $\mathcal{A}$, which is a subset of the set of (equivalence classes of) irreducible bounded representations of $\mathfrak{g}$. In this sense, Theorem 4.11 actually leads to a classification of all bounded unitary representations of $\mathfrak{g}$, not just of the irreducible ones.

We now consider the Lie algebra $\mathfrak{g}=\Gamma_{c}(\mathfrak{K})$ for a non-compact manifold $X$. For every bounded factor representation $(\pi, \mathcal{H})$ of $\mathfrak{g}$ and every compact equidimensional submanifold $Y \subseteq X$ with boundary, we have seen in Theorem 4.10 that there exists a factorization $\pi=\pi_{1} \otimes \pi_{2}$ for which $\Gamma(\mathfrak{K})_{Y} \subseteq \operatorname{ker} \pi_{1}$, and $\pi_{2}$ is a finite tensor product of evaluation representations $\bigotimes_{X \in X} \rho_{X} \circ \operatorname{ev}_{X}$, where $\left(\rho_{X}, V_{X}\right)$ are irreducible representations of the Lie algebras $\mathfrak{K}_{x} \cong \mathfrak{k}$. Therefore,

$$
C^{*}\left(\pi\left(\Gamma(\mathfrak{K})_{Y}\right)\right) \cong C^{*}\left(\pi_{2}\left(\Gamma(\mathfrak{K})_{Y}\right)\right) .
$$

We may then assume w.l.o.g. that $\mathbf{x} \subseteq Y^{0}$, which further implies that $\pi_{2}\left(\Gamma(\mathfrak{K})_{Y}\right) \cong$ $\bigoplus_{x \in \mathbf{X}} \rho_{X}\left(\mathfrak{K}_{X}\right)$, and thus

$$
C^{*}\left(\pi_{2}\left(\Gamma(\mathfrak{K})_{Y}\right)\right) \cong \bigotimes_{X \in \mathbf{X}} B\left(V_{X}\right)
$$

Since $X$ is $\sigma$-compact, $X=\bigcup_{n} Y_{n}$ with $Y_{n} \subseteq Y_{n+1}^{0}$ and $Y_{n}$ is a compact submanifold with boundary, so we can iterate the preceding construction. Therefore, $\Gamma_{c}(\mathfrak{K})=$ $\bigcup_{n} \Gamma(\mathfrak{K})_{Y_{n}}$ implies the existence of a locally finite subset $\mathbf{x} \subseteq X$ such that

$$
C^{*}\left(\pi\left(\Gamma_{C}(\mathfrak{K})\right)\right) \cong \lim _{\longrightarrow} C^{*}\left(\pi\left(\Gamma(\mathfrak{K})_{Y_{n}}\right)\right) \cong \lim _{\longrightarrow} \bigotimes_{x \in \mathbf{x} \cap Y_{n}^{0}} B\left(V_{X}\right)=: \widehat{\bigotimes}_{x \in \mathbf{X}} B\left(V_{X}\right),
$$

where the second limit is the direct limit of the net of $C^{*}$-algebras $\bigotimes_{X \in \mathbf{X} \cap Y_{n}^{0}} B\left(V_{X}\right)$ over the directed system of finite subsets $\mathbf{x} \cap Y_{n}^{0}$ of $\mathbf{x}$, that is, the norm completion of the algebraic limit. Defining

$$
\mathcal{A}_{\mathbf{x}, \rho}:=\widehat{\bigotimes}_{x \in \mathbf{x}} B\left(V_{x}\right),
$$

we obtain for every $x$ a canonical inclusion $\iota_{X}: B\left(V_{X}\right) \hookrightarrow \mathcal{A}_{\mathbf{x}, \rho}$. 
Conversely, for every locally finite subset $\mathbf{x} \subseteq X$ with corresponding irreducible unitary representations $\rho_{X}$ of $\mathfrak{K}_{X}$, we obtain a Lie algebra homomorphism

$$
\eta_{\mathbf{X}, \rho}: \Gamma_{C}(\mathfrak{K}) \rightarrow \mathcal{A}_{\mathbf{X}, \rho}, \quad s \mapsto \sum_{X \in \mathbf{X}} \iota_{X} \circ \rho_{X}(s(X))
$$

whose image generates a dense subalgebra. (The sum only has finitely many terms.)

The preceding discussion now leads to the following theorem which describes the bounded irreducible and factor representations of the LF-Lie algebra $\Gamma_{C}(\mathfrak{K})$ in terms of irreducible representations of the $C^{*}$-algebras $\mathcal{A}_{\mathbf{x}, \rho}$. It reduces all Lie theoretic issues concerning these representation to questions concerning $C^{*}$-algebras.

Theorem 4.13. Suppose that $X$ is a smooth manifold with compact boundary and that $\mathfrak{K} \rightarrow X$ is a smooth Lie algebra bundle whose typical fiber $\mathfrak{k}$ is a compact semisimple Lie algebra. For every bounded unitary factor (irreducible) representation $(\pi, \mathcal{H})$ of $\Gamma_{c}(\mathfrak{K})$, there exists a locally finite subset $\mathbf{x} \subseteq X$, irreducible representations $\rho_{X}$ of $\mathfrak{K}_{X}$ corresponding to $x \in \mathbf{x}$, and a unique factor (irreducible) representation $\beta: \mathcal{A}_{\mathbf{x}, \rho} \rightarrow B(\mathcal{H})$ with $\pi \simeq \beta \circ \eta_{\mathbf{x}, \rho}$. Conversely, every representation of the type $\left(\beta \circ \eta_{\mathbf{x}, \rho}, \mathcal{H}\right)$ is a bounded factor (irreducible) representation. Two such representations are equivalent, $\beta \circ \eta_{\mathbf{x}, \rho} \simeq \beta^{\prime} \circ \eta_{\mathbf{x}^{\prime}, \rho^{\prime}}$, if and only if $\mathbf{x}=\mathbf{x}^{\prime}, \rho_{X} \simeq \rho_{X}^{\prime}$ for all $x \in \mathbf{x}$, and $\beta \simeq \beta^{\prime}$.

Remark 4.14. The algebra $\mathcal{A}_{\mathbf{x}, \rho}$ is a so-called UHF (ultra hyperfinite) $C^{*}$-algebra. These algebras have been classified by Glimm in [17], where one also finds a characterization of their pure states. Even stronger results were obtained later by Powers in [38], where he shows that the automorphism group acts transitively on the set of pure states, so that every irreducible representation is a twist (by an automorphism) of an infinite tensor product of irreducible representations. Together with these results, Theorem 4.13 provides a complete description of the bounded irreducible unitary representations of $\Gamma_{C}(\mathfrak{K})$.

Example 4.15. If $\mathcal{A}=\widehat{\bigotimes}_{x \in \mathrm{x}} B\left(V_{X}\right)$ is an UHF $C^{*}$-algebra, then typical examples of irreducible representations are the infinite tensor products $\widehat{\bigotimes}_{X \in \mathbf{X}}\left(V_{X}, v_{X}\right)$, where $v_{X} \in V_{X}$ is a unit vector. All these representations are irreducible ([44, Proposition 5.2.1]; see also [43, Proposition 4.4.3]), but they do not exhaust all irreducible representations. The restriction of these representations to the canonical maximal abelian subalgebras is multiplicity free. This is not true for all irreducible unitary representations (cf. [44, Sect. 5.2]). Two such infinite tensor product representations corresponding to the sequences $\left(v_{x}\right)$ 
and $\left(w_{x}\right)$ of unit vectors are unitarily equivalent if and only if

$$
\sum_{X \in \mathbf{X}} 1-\left|\left\langle v_{X}, w_{X}\right\rangle\right|<\infty
$$

[44, Proposition5.2.2]. In particular, there exist infinitely many non-equivalent irreducible unitary representations. The above condition is equivalent to

$$
\sum_{x \in \mathbf{X}}^{\infty} d\left(\left[v_{X}\right],\left[w_{X}\right]\right)^{2}<\infty
$$

since the natural metric on the projective space $\mathbb{P}\left(V_{X}\right)$ satisfies $d([v],[w])^{2}=2(1-|\langle v, w\rangle|)$ [33, Lemma 3.2].

\subsection{Translation to the group context}

We have carried out our classification of the bounded unitary representations on the Lie algebra level, which is equivalent to working with the corresponding 1-connected groups. However, some natural mapping groups, such as $G:=C^{\infty}(X, K)$, where $X$ is compact (take f.i. $X=\mathbb{S}^{3}$ ) and $K$ is a 1-connected compact group, are neither connected nor simply connected.

Let $P \rightarrow X$ be a principal $K$-bundle over a compact space $X$, with $K$ compact semisimple. Since every irreducible bounded representation of $\Gamma(\operatorname{Ad}(P))$ is a finite tensor product of irreducible evaluation representations and every irreducible evaluation representation obviously integrates to a representation of $\mathrm{Gau}(P)$, all bounded unitary representations do. They actually factor through quotient homomorphisms $\mathrm{Gau}(P) \rightarrow$ $\Pi_{X \in \mathrm{X}} \mathrm{Gau}\left(\left.P\right|_{X}\right)$, given by evaluation in the finite subset $\mathbf{x} \subseteq X$. Each factor $\operatorname{Gau}\left(\left.P\right|_{X}\right)$ is isomorphic to $K$, so $\Pi_{X \in \mathrm{X}} \mathrm{Gau}\left(\left.P\right|_{X}\right)$ is isomorphic to the 1 -connected group $K^{\mathrm{x}}$. In particular, the group $\pi_{0}(\mathrm{Gau}(P))$ acts trivially on the set $\hat{\mathrm{Gau}}(P)_{0}{ }^{b}$ of equivalence classes of bounded irreducible unitary representations of the identity component $\mathrm{Gau}(P)_{0}$. If $\mathrm{Gau}(P)$ is not connected, then a bounded irreducible unitary representation is not determined by its derived Lie algebra representation. In this context, however, we do have the following theorem:

Theorem 4.16. Every irreducible bounded unitary representation of $\mathrm{Gau}(P)$ is equivalent to $\pi_{1} \otimes \pi_{2}$, where $\pi_{1}$ is a finite tensor product of irreducible evaluation representations at different points, and $\pi_{2}$ comes from an irreducible unitary representation of the discrete group $\pi_{0}(\mathrm{Gau}(P))$. Conversely, any such tensor product is irreducible. 
Proof. We set $G:=\operatorname{Gau}(P)$. Let $\mathcal{A}:=C^{*}\left(\pi\left(G_{0}\right)\right)=C^{*}(\mathrm{~d} \pi(\mathfrak{g})) \subseteq B(\mathcal{H})$ denote the $C^{*}$-algebra generated by $\pi\left(G_{0}\right)$. Then every irreducible representation $\beta$ of $\mathcal{A}$ defines a bounded unitary representation of $G_{0}$, hence is finite dimensional. In particular, the image of $\mathcal{A}$ in every irreducible representation contains the compact operators, so that $\mathcal{A}$ is type I [42]. The group $G$ acts by conjugation on $\mathcal{A}$ but we have already seen above that its action on the space $\hat{\mathcal{A}}$ of equivalence classes of irreducible representations of $\mathcal{A}$ is trivial.

Since $\mathcal{H}$ and $\mathcal{A}$ are separable and $\mathcal{A}$ is of type I, the representation of $\mathcal{A}$ on $\mathcal{H}$ has a canonical direct integral decomposition

$$
\mathcal{H} \cong \int_{\hat{\mathcal{A}}}^{\oplus} \mathcal{H}_{\alpha} \mathrm{d} \mu(\alpha) \cong \bigoplus_{n \in \mathbb{N} \cup\{\infty\}} \int_{\hat{\mathcal{A}}_{n}} \mathcal{H}_{\alpha} \mathrm{d} \mu_{n}(\alpha)
$$

for a measure $\mu$ on $\hat{\mathcal{A}}$ which is a sum of disjoint measures $\mu_{n}:=\left.\mu\right|_{\hat{\mathcal{A}}_{n}}, n \in \mathbb{N} \cup\{\infty\}$, where $\left(\hat{\mathcal{A}}_{n}\right)_{n \in \mathbb{N} \cup\{\infty\}}$ is a measurable partition of $\hat{\mathcal{A}}$, and for which the representation on $\mathcal{H}_{\alpha}$, $\alpha \in \hat{\mathcal{A}}_{n}$, is an $n$-fold multiple of an irreducible representation of type $\alpha$ [11, Theorem 8.6.6]. Here the measure classes $\left[\mu_{n}\right], n \in \mathbb{N} \cup\{\infty\}$, are uniquely determined by the representation. Since $G$ acts trivially on $\hat{\mathcal{A}}$, it preserves all these measure classes. Therefore, the irreducibility of the representation implies that only one of these measures is non-zero, and that this measure is ergodic for the $G$-action on $\hat{\mathcal{A}}$, hence a point measure because the action is trivial. Therefore, $\left.\pi\right|_{G_{0}}$ is a factor representation of type $I$, hence a multiple of a tensor product of irreducible evaluation representations at different points. This implies that $\pi \cong \pi_{1} \otimes \pi_{2}$, where $\pi_{1}$ is a finite tensor product of irreducible evaluation representations at different points, and $\pi_{2}$ vanishes on $G_{0}$, hence defines an irreducible unitary representation of the discrete group $\pi_{0}(G)$. Conversely, any such tensor product is irreducible.

\section{Noncompact Fibers and Projective Representations}

In this section, we show that the problem of classifying bounded irreducible projective unitary representations $\bar{\pi}: \Gamma_{C}(\mathfrak{K}) \rightarrow \mathfrak{p u}(\mathcal{H})$, where the typical fiber $\mathfrak{k}$ of $\mathfrak{K}$ is an arbitrary finite-dimensional real Lie algebra, reduces to Theorems 4.11 and 4.13. This justifies our assumptions that $\mathfrak{k}$ is compact semisimple and that $\pi: \Gamma_{C}(\mathfrak{K}) \rightarrow \mathfrak{u}(\mathcal{H})$ is a linear representation.

We start with the observation that a finite-dimensional Lie algebra $\mathfrak{k}$ is compact if and only if its adjoint group $\left\langle\mathrm{e}^{\mathrm{adk}}\right\rangle$ is relatively compact in GL(k), which in turn is equivalent to the existence of an adjoint invariant norm on $\mathfrak{k}$. Next we observe that every finite-dimensional Lie algebra $\mathfrak{k}$ contains a unique minimal ideal $\mathfrak{n} \unlhd \mathfrak{k}$ for which $\mathfrak{k} / \mathfrak{n}$ is 
compact. This is a direct consequence of the fact that direct sums and subalgebras of compact Lie algebras are compact, which implies that the set of all ideals with compact quotients is filtered.

Proposition 5.1. Let $\mathcal{A}$ be a real commutative associative locally convex algebra and let $p$ be a bounded seminorm on $\mathfrak{k} \otimes \mathcal{A}$ which is invariant under $\mathrm{e}^{\text {adk }}$. Let $\mathfrak{n} \unlhd \mathfrak{k}$ be the minimal ideal for which $\mathfrak{k} / \mathfrak{n}$ is a compact Lie algebra. Then $\mathfrak{n} \otimes \mathcal{A} \subseteq p^{-1}(0)$.

Proof. Let $\mathfrak{g}=\mathfrak{k} \otimes \mathcal{A}$. For each $a \in \mathcal{A}$, we consider the seminorm $p_{a}(x):=p(x \otimes a)$ on $\mathfrak{k}$. Since the map $\mathfrak{k} \rightarrow \mathfrak{k} \otimes \mathcal{A}, x \mapsto x \otimes a$ is $\mathfrak{k}$-equivariant, the seminorm $p_{a}$ on $\mathfrak{k}$ is invariant. Therefore, $p_{a}$ induces on the quotient $\mathfrak{k} / p_{a}^{-1}(0)$ an invariant norm, so that this Lie algebra is compact. This implies that $\mathfrak{n} \subseteq p_{a}^{-1}(0)$, and hence that $\mathfrak{n} \otimes \mathcal{A} \subseteq p^{-1}(0)$.

Corollary 5.2. Let $p$ be a bounded seminorm on $C^{\infty}(X, \mathfrak{k})$ or $C_{c}^{\infty}(X, \mathfrak{k})$, which is invariant under the adjoint action and let $\mathfrak{n} \unlhd \mathfrak{k}$ be the minimal ideal for which $\mathfrak{k} / \mathfrak{n}$ is a compact Lie algebra. Then $C^{\infty}(X, \mathfrak{n})$ and $C_{c}^{\infty}(X, \mathfrak{n})$, respectively, are contained in the closed ideal $p^{-1}(0)$.

Proof. The Lie algebra $C^{\infty}(X, \mathfrak{k})$ is covered by the preceding proposition because $C^{\infty}(X, \mathbb{R})$ is unital, so that $\mathfrak{k} \cong \mathfrak{k} \otimes 1 \subseteq \mathfrak{k} \otimes \mathcal{A}$.

For $\mathcal{A}=C_{c}^{\infty}(X, \mathbb{R})$, we fix a compact subset $Y \subseteq X$ and consider the subalgebra

$$
\mathcal{A}_{Y}:=C^{\infty}(X, \mathbb{R})_{Y}:=\left\{f \in C^{\infty}(X, \mathbb{R}): \operatorname{supp}(f) \subseteq Y\right\} .
$$

Let $\chi \in C_{c}^{\infty}(X, \mathbb{R})$ with $\left.\chi\right|_{Y}=1$. For $X \in \mathfrak{k}$, we then have

$$
\operatorname{ad}(x \otimes \chi)(y \otimes a)=[x, y] \otimes a \quad \text { for } a \in \mathcal{A}_{Y} .
$$

Therefore, the restriction of $p$ to $\mathfrak{k} \otimes \mathcal{A}_{Y}$ is invariant under $\mathrm{e}^{\mathrm{adk}}$, so that the assertion follows from Proposition 5.1.

Corollary 5.3. Let $\mathfrak{K} \rightarrow X$ be a $\mathfrak{k}$-Lie algebra bundle, where $\mathfrak{k}$ is a finite-dimensional real Lie algebra, let $\mathfrak{n} \unlhd \mathfrak{k}$ be the minimal ideal for which $\mathfrak{k} / \mathfrak{n}$ is a compact Lie algebra, and let $\mathfrak{N} \subseteq \mathfrak{K}$ be the corresponding subbundle. If $\bar{\pi}: \Gamma_{C}(\mathfrak{K}) \rightarrow \mathfrak{p u}(\mathcal{H})$ is a projective unitary representation, then $\Gamma_{C}(\mathfrak{N}) \subseteq \operatorname{ker} \bar{\pi}$.

Proof. Since the operator norm on $\mathfrak{u}(\mathcal{H})$ is invariant under the adjoint action of $\mathrm{U}(\mathcal{H})$, it induces on the Banach-Lie algebra $\mathfrak{p u}(\mathcal{H})=\mathfrak{u}(\mathcal{H}) / i \mathbb{R} 1$ a norm which is also invariant 
under the adjoint action. Therefore, $p(s):=\|\bar{\pi}(s)\|$ is an invariant seminorm on $\Gamma_{c}(\mathfrak{K})$. We have to show that $\Gamma_{c}(\mathfrak{N}) \subseteq p^{-1}(0)$.

In view of Remark $4.5, \Gamma_{C}(\mathfrak{K})$ is a sum of ideals of the form $\Gamma_{C}\left(\left.\mathfrak{K}\right|_{U}\right) \cong C_{C}^{\infty}(U, \mathfrak{k})$, where $\left.\mathfrak{K}\right|_{U}$ is trivial. It therefore suffices to observe that Corollary $5.2 \operatorname{implies} C_{C}^{\infty}(U, \mathfrak{n}) \cong$ $\Gamma_{C}\left(\left.\mathfrak{N}\right|_{U}\right) \subseteq p^{-1}(0)$.

The preceding corollary shows that, for the sake of classifying bounded projective unitary representations of the Lie algebra $\Gamma_{C}(\mathfrak{K})$, we may w.l.o.g. assume that $\mathfrak{k}$ is compact. As the following theorem shows, the corresponding cocycles must be trivial.

Theorem 5.4. If $\mathfrak{K}$ is a bundle of Lie algebras with compact fiber, then every bounded projective unitary representation of $\Gamma_{C}(\mathfrak{K})$ lifts to a bounded unitary representation.

Proof. Let $\pi: \Gamma_{c}(\mathfrak{K}) \rightarrow \mathfrak{u}(\mathcal{H})$ be a bounded projective representation. Then there exists a cocycle $\omega: \Gamma_{C}(\mathfrak{K}) \times \Gamma_{C}(\mathfrak{K}) \rightarrow \mathbb{R}$ such that $\pi$ is a unitary representation of $\mathbb{R} C \oplus_{\omega} \Gamma_{C}(\mathfrak{K})$ mapping $c$ to $i 1$. As the decomposition $\mathfrak{k}=\mathfrak{z}(\mathfrak{k}) \oplus[\mathfrak{k}, \mathfrak{k}]$ is $\operatorname{Aut}(\mathfrak{k})$-invariant, it leads to a corresponding direct sum $\mathfrak{K}=Z(\mathfrak{K}) \oplus[\mathfrak{K}, \mathfrak{K}]$ of Lie algebra bundles, and hence to $\Gamma_{c}(\mathfrak{K})=$ $\Gamma_{C}(\mathfrak{z}(\mathfrak{K})) \oplus \Gamma_{C}([\mathfrak{K}, \mathfrak{K}])$.

First of all, we show that $\omega$ vanishes on $\Gamma_{C}(\mathfrak{z}(\mathfrak{K})) \times \Gamma_{C}(\mathfrak{z}(\mathfrak{K}))$. Indeed, for all $z, z^{\prime} \in \Gamma_{C}(\mathfrak{z}(\mathfrak{K})), \pi$ defines a bounded representation of the at most two-step nilpotent Lie algebra spanned by $z, z^{\prime}$, and $c$, and thus vanishes on $\left[z, z^{\prime}\right]=\omega\left(z, z^{\prime}\right) c$. This implies that $\omega\left(z, z^{\prime}\right)=0$. Furthermore, the cocycle property implies that $\omega(z,[\xi, \eta])=0$ for all $z \in \Gamma_{C}(\mathfrak{z}(\mathfrak{K}))$ and $\xi, \eta \in \Gamma_{C}(\mathfrak{K})$, so that we may assume w.l.o.g. that $\mathfrak{k}$ is compact semisimple.

Let $\mathbb{V}:=S^{2}(\mathfrak{K}) /\left\langle\operatorname{ad}(\mathfrak{K}) \cdot S^{2}(\mathfrak{K})\right\rangle, \kappa: \mathfrak{K} \times \mathfrak{K} \rightarrow \mathbb{V}$ be the universal invariant symmetric bilinear bundle map. Then $\mathbb{V}$ is a flat bundle, hence carries a canonical differential d: $\Gamma(\mathbb{V}) \rightarrow \Omega^{1}(X, \mathbb{V})$. We know from [27] that there exists a Lie connection $\nabla$ on $\mathfrak{K}$ and a continuous linear functional $\lambda$ on $\Omega_{c}^{1}(M, \mathbb{V})$ vanishing on $\mathbf{d} \Gamma_{c}(\mathbb{V})$, such that $\omega$ is cohomologous to the cocycle $(\xi, \eta) \mapsto \lambda(\kappa(\xi, \nabla \eta))$. Suppose that $\lambda \neq 0$. Then there exists a $\gamma \in \Omega_{c}^{1}(M, \mathbb{V})$ with $\lambda(\gamma) \neq 0$. We may assume w.l.o.g. that $\gamma$ is supported in an open set $U \subset M$ over which $\mathfrak{K}$ (and thus $\mathbb{V}$ ) can be trivialized. Using this trivialization, we write $\gamma=\sum_{j} f_{j} \mathbf{d} g_{j} \otimes \kappa\left(X_{j}, Y_{j}\right)$. By polarization, we may assume that $X_{j}=Y_{j}$, so that we find $f \otimes X$ and $g \otimes X$ with $\lambda(\kappa(f \otimes X, \nabla g \otimes X)) \neq 0$. As $\pi$ defines a bounded unitary representation of the two-step nilpotent Lie algebra spanned by $f \otimes X, g \otimes X$, and $c$, we must have $\lambda(\kappa(f \otimes X, \nabla g \otimes X))=0$, contradicting our hypothesis. This means that $\omega$ is a coboundary, and $\pi$ lifts to a bounded unitary representation of $\Gamma_{C}(\mathfrak{K})$.

By Remark 4.2, we may assume w.l.o.g. that $\mathfrak{k}$ is compact semisimple, which renders Theorems 4.11 and 4.13 applicable. 


\section{Boundary Conditions and Non-Unital Algebras}

In this final section, we discuss Lie algebras of the form $\mathfrak{k}_{\mathcal{A}}=\mathfrak{k} \otimes_{\mathbb{R}} \mathcal{A}_{\mathbb{R}}$ for $\mathcal{A}$ non-unital, and Lie algebras of sections of $\mathfrak{K}$ that satisfy vanishing conditions at the boundary of $X$.

First we show that, for $\mathcal{A}=\ell^{1}(\mathbb{N}, \mathbb{C})$ (with the pointwise product), the Lie algebra $\mathfrak{k}_{\mathcal{A}}$ has infinite-dimensional bounded irreducible representations (Section 6.1). We already know from Section 4 that this is the case for $\mathcal{A}=C_{C}^{\infty}(X, \mathbb{R})$, where $X$ is a noncompact manifold, but the case of $\ell^{1}(\mathbb{N}, \mathbb{C})$ shows that this also happens for Banach algebras. In view of this observation, it is remarkable that this phenomenon does not occur for non-unital $C^{*}$-algebras, as we show in Section 6.2. Given these two classes of examples, one expects that, for a Banach algebra $\mathcal{A}$ with $\ell^{1}(\mathbb{N}, \mathbb{C}) \subseteq \mathcal{A} \subseteq c_{0}(\mathbb{N}$, $\mathbb{C})$, a mixture of the "tame" behavior of $c_{0}(\mathbb{N}, \mathbb{C})$, where all irreducible representations are finite dimensional, and the "wild" behavior for $\ell^{1}(\mathbb{N}, \mathbb{C})$ will occur.

This issue is addressed on a quantitative level in Section 6.3, where we discuss the Banach-Lie algebra $\Gamma_{0}^{k}(\mathfrak{K})$ of $C^{k}$-sections of $\mathfrak{K}$ whose $k$-jet vanishes at the boundary $\partial X$ of $X$. This is a Banach completion of $\Gamma_{C}\left(X^{\circ}\right)$ and we characterize those pairs $(\mathbf{x}, \rho)$ for which the homomorphism $\eta_{\mathbf{x}, \rho}: \Gamma_{C}(\mathfrak{K}) \rightarrow \mathcal{A}_{\mathbf{x}, \rho}$ extends continuously to $\Gamma_{0}^{k}(\mathfrak{K})$.

\subsection{Bounded representations of $\ell^{1}(\mathbb{N}, \mathfrak{k})$}

The simplest non-compact manifold is $X=\mathbb{N}$. In this case

$$
C_{C}^{\infty}(X, \mathfrak{k}) \cong \mathfrak{k}^{(\mathbb{N})}:=\lim _{\longrightarrow} \mathfrak{k}^{N}:=\left\{X=\left(X_{n}\right) \in \mathfrak{k}^{\mathbb{N}}\left|\left\{n \in \mathbb{N}: X_{n} \neq 0\right\}\right|<\infty\right\}
$$

From Theorem 4.13, it follows that $\mathfrak{k}^{(\mathbb{N})}$ has a wild bounded unitary representation theory. That this is not a phenomenon caused by the rather fine topology on this Lie algebra, follows from the fact that it is shared by certain Banach completions. Let $\rho:=\left(\rho_{n}, V_{n}\right)_{n \in \mathbb{N}}$ be a sequence of irreducible unitary representations of $\mathfrak{k}$ and let $\mathcal{A}_{\rho}:=\widehat{\bigotimes}_{n \in \mathbb{N}} B\left(V_{n}\right)$ denote the corresponding UHF $C^{*}$-algebra. We further assume that sup $\left\|\rho_{n}\right\|<\infty$. Then the inclusion $\mathfrak{k}^{(\mathbb{N})} \rightarrow \mathcal{A}_{\rho}$ extends to a continuous embedding

$$
\eta_{\rho}: \mathfrak{g}:=\ell^{1}(\mathbb{N}, \mathfrak{k}) \rightarrow \mathcal{A}_{\rho}, \quad\left(X_{n}\right)_{n \in \mathbb{N}} \mapsto \sum_{n=1}^{\infty} \mathbf{1}^{\otimes(n-1)} \otimes \rho_{n}\left(X_{n}\right) \otimes \mathbf{1}^{\otimes \infty}
$$

with $\left\|\eta_{\rho}\right\| \leq \sup _{n \in \mathbb{N}}\left\|\rho_{n}\right\|$. Since $\eta_{\rho}$ maps $\mathfrak{k}^{(\mathbb{N})}$ to a topologically generating subalgebra, $\operatorname{im}\left(\eta_{\rho}\right)$ generates a dense subalgebra. Therefore, the Banach-Lie algebra $\mathfrak{g}$ has bounded factor representations of types II and III. 
Example 6.1 (cf. [43, pp. 205 et seqq.]). For $\mathfrak{k}=\mathfrak{s u}_{2}(\mathbb{C})$ and the defining representation $\rho_{n}: \mathfrak{k} \rightarrow M_{2}(\mathbb{C})$, we consider the algebra $\mathcal{A}:=\mathcal{A}_{\rho}$, that is, $\mathcal{A}=\widehat{\bigotimes}_{n \in \mathbb{N}} M_{2}(\mathbb{C})$. Let $0 \leq p_{n} \leq \frac{1}{2}$ and consider the factorial state

$$
\phi_{n}\left(\begin{array}{ll}
a & b \\
c & d
\end{array}\right):=p_{n} a+\left(1-p_{n}\right) d
$$

on $M_{2}(\mathbb{C})$. Then $\psi:=\bigotimes_{n \in \mathbb{N}} \phi_{n}$ is a factorial state on $\mathcal{A}$. If $p_{n}=0$ for every $n$, then $\psi$ is pure, so that we obtain a type $\mathrm{I}_{\infty}$ representation. If $p_{n}=\frac{1}{2}$ for every $n$, then $\pi_{\psi}(\mathcal{A})^{\prime \prime}$ is a type $\mathrm{II}_{1}$-factor. If there exists a $\delta>0$ with $\delta<p_{n}<\frac{1}{2}-\delta$ for every $n$, then $\pi_{\psi}(\mathcal{A})^{\prime \prime}$ is a type III factor. For $\left.p_{n}=\lambda \in\right] 0, \frac{1}{2}\left[, n \in \mathbb{N}\right.$, the factor $\mathcal{M}_{\lambda}:=\pi_{\psi}(\mathcal{A})^{\prime \prime}$ is called a Powers factor (cf. [38]).

Remark 6.2. It is easy to see that the map $\eta_{\rho}$ actually is an isometric embedding $\ell^{1}(\mathbb{N}, \mathfrak{k}) \hookrightarrow \mathcal{A}$. Let $\beta: \mathcal{A} \rightarrow B(\mathcal{H})$ be a factor representation of $\mathcal{A}$. Since $\mathcal{A}$ is simple, $\beta$ is isometric, and this implies for every sequence $\left(X_{n}\right) \in \mathfrak{k}^{\mathbb{N}}$ that

$$
\lim _{N \rightarrow \infty}\left\|\beta \circ \eta_{\rho}\left(X_{1}, X_{2}, \ldots, X_{N}, 0, \cdots\right)\right\|=\sum_{n}\left\|X_{n}\right\|
$$

In particular, the representation $\pi:=\beta \circ \eta_{\rho}$ does not extend to a bounded representation of $\ell^{2}(\mathbb{N}, \mathfrak{k})$, or any other Banach-Lie algebra containing $\ell^{1}(\mathbb{N}, \mathfrak{k})$ as a proper dense subspace.

As the spectra of elements in $\ell^{1}(\mathbb{N}, \mathfrak{k})$ are symmetric, it follows that the map

$$
\bar{\eta}_{\rho}: \ell^{1}(\mathbb{N}, \mathfrak{k}) \rightarrow \operatorname{der}(\mathcal{A}), \quad X \mapsto \operatorname{ad}\left(\eta_{\rho}(X)\right)
$$

is isometric. Therefore, even the bounded projective representation $\bar{\pi}: \ell^{1}(\mathbb{N}, \mathfrak{k}) \rightarrow \mathfrak{p u}(\mathcal{H})$ does not extend to any Banach-Lie algebra containing $\ell^{1}(\mathbb{N}, \mathfrak{k})$ as a proper dense subspace.

However, one can show that for certain $\beta, \pi$ has a unique proper extension to a projective unitary representation of $\ell^{2}(\mathbb{N}, \mathfrak{k})$ by unbounded operators and that this representation integrates to an analytic representation of a non-trivial central $\mathbb{T}$-extension of the corresponding Banach-Lie group

$$
\ell^{2}\left(\mathbb{N}, \mathrm{SU}_{2}(\mathbb{C})\right):=\left\{k \in \mathrm{SU}_{2}(\mathbb{C})^{\mathbb{N}}: \sum_{n=1}^{\infty}\left\|\mathbf{1}-k_{n}\right\|^{2}<\infty\right\}
$$

We shall explore this and related phenomena in subsequent work (cf. [26]). 
Example 6.3. Note that any Lie algebra that has a dense continuous homomorphism into $\ell^{1}(\mathbb{N}, \mathfrak{k})$ will inherit the "wild" factor representations mentioned in Example 6.1. For example, the Lie algebra $\mathcal{S}\left(\mathbb{R}^{d}, \mathfrak{k}\right)$ of $\mathfrak{k}$-valued Schwartz functions allows for the dense homomorphism $\mathcal{S}\left(\mathbb{R}^{d}, \mathfrak{k}\right) \rightarrow \ell^{1}(\mathbb{N}, \mathfrak{k})$ defined by $f \mapsto\left(f\left(n e_{1}\right)\right)_{n \in \mathbb{N}}$.

Example 6.4. Consider the Lie algebra $\mathfrak{k}_{\mathcal{A}}:=\mathfrak{k} \otimes_{\mathbb{R}} \mathcal{A}_{\mathbb{R}}$, where $\mathcal{A}:=C_{0}^{k}([0,1], \mathbb{C})$ is the commutative Banach algebra

$$
C_{0}^{k}([0,1], \mathbb{C}):=\left\{f \in C^{k}([0,1], \mathbb{C}): f^{(j)}(0)=0, \forall j \in\{0, \ldots, k\}\right\}
$$

If $k \geq 1$, then we have a dense continuous Lie algebra homomorphism into $\ell^{1}(\mathbb{N}, \mathfrak{k})$, derived from the Banach algebra homomorphism $\eta: \mathcal{A} \rightarrow \ell^{1}(\mathbb{N}, \mathbb{C})$ defined by $\eta(f)_{n}=$ $f\left(x_{n}\right)$, with $x_{n}:=\frac{1}{(n+1)^{2}}$. It is continuous because $|f(x)| \leq \frac{1}{k !} x^{k}\left\|f^{(k)}\right\|_{\infty}$, and $1 /(n+1)^{2 k}$ is summable for $k \geq 1$. Consequently, $\mathfrak{K}_{\mathcal{A}}$ has bounded unitary factor representations of types II and III if $k \geq 1$. However, we will see in Section 6.2 that, for $k=0$, all irreducible bounded unitary representations are finite dimensional, so that in this case, the bounded unitary factor representations are all of type I.

In the following, we will perform a more refined analysis of Example 6.4. In Section 6.2 below, we will show that if $\mathcal{A}:=C_{0}(X, \mathbb{C})$ is a (possibly non-unital) $C^{*}$-algebra, then every irreducible bounded unitary representation of $\mathfrak{k}_{\mathcal{A}}$ is a finite tensor product of evaluation representations. Note that the Banach algebra $C_{0}^{k}([0,1], \mathfrak{k})$ is a $C^{*}$-algebra only if $k=0$. This is what causes the "tame" behavior that distinguishes it from its siblings with $k \geq 1$. In Section 6.3, we will take up the thread for $k \geq 1$. We will show, again in a more general context, that an infinite evaluation representation in a sequence $\left(x_{n}\right)$ is defined if and only if $x_{n}^{k}$ is summable.

\subsection{Non-unital $C^{*}$-algebras}

Let $\mathcal{A} \cong C_{0}(X)$ be the $C^{*}$-algebra of continuous functions that vanish at infinity, for $X$ a locally compact space which is not compact. The dual $\mathcal{A}_{\mathbb{R}}^{\prime}$ of the Banach space $\mathcal{A}_{\mathbb{R}} \cong$ $C_{0}(X, \mathbb{R})$ can be identified with the space $\mathcal{M}(X)$ of finite regular Borel measures on $X$. Let $B \subseteq \mathcal{A}_{\mathbb{R}}^{\prime}$ be a weak-*-compact subset. Then we have a natural map $\mathcal{A}_{\mathbb{R}} \rightarrow C(B, \mathbb{R}), a \mapsto a^{*}$ with $a^{*}(\alpha):=\alpha(a)$.

Lemma 6.5. If $\left(\delta_{n}\right)_{n \in \mathcal{A}}$ is an approximate identity in $\mathcal{A}$ with $0 \leq \delta_{n} \leq 1$, then $\delta_{n}^{*} \rightarrow 1^{*}$ holds pointwise on $\mathcal{A}^{\prime}$. 
Proof. If $\mu \in \mathcal{A}^{\prime} \cong \mathcal{M}(X)$ is a complex regular measure on $X$, then we have to show that

$$
\int_{X} \delta_{n} \mathrm{~d} \mu \rightarrow \int_{X} 1 \mathrm{~d} \mu=\mu(X) .
$$

Since $\mu$ is a linear combination of four positive measures [40, Theorem 6.14], we may w.l.o.g. assume that $\mu$ is positive. Let $\varepsilon>0$ and pick a compact subset $K \subseteq X$ with $\mu(X \backslash K) \leq \varepsilon$. There exists a function $\chi \in C_{0}(X)$ with $\left.\chi\right|_{K}=1$. Then $\delta_{n} \chi \rightarrow \chi$ implies that $\left.\delta_{n}\right|_{K}$ converges uniformly to 1 , so that $\int_{K} \delta_{n} \mathrm{~d} \mu \rightarrow \mu(K)$. Since

$$
0 \leq \int_{X \backslash K} \delta_{n} \mathrm{~d} \mu \leq \mu(X \backslash K) \leq \varepsilon
$$

it follows that, eventually, $\left|\int_{X} \delta_{n} \mathrm{~d} \mu-\mu(X)\right| \leq 2 \varepsilon$.

Remark 6.6. A sequence $\left(\delta_{n}\right)_{n \in \mathbb{N}}$ with the above properties exists if and only if $X$ is countable at infinity. In fact, if $X$ is countable at infinity, then there exists an exhaustion $\left(K_{n}\right)_{n \in \mathbb{N}}$ by compact subsets satisfying $K_{n} \subseteq K_{n+1}^{0}$. Then Urysohn's lemma implies the existence of $\delta_{n} \in C_{0}(X)$ with $\left.\delta_{n}\right|_{K_{n}}=1$ and $0 \leq \delta_{n} \leq 1$. Now $\left(\delta_{n}\right)$ is an approximate identity of $C_{0}(X)$.

If, conversely, $\left(\delta_{n}\right)$ exists, then we consider the compact subsets $K_{n}:=\left\{\delta_{n} \geq \frac{1}{n}\right\} \subseteq$ $X$. Since $\delta_{n} \rightarrow 1$ holds uniformly on every compact subset $K \subseteq X$, there exists an $n \in \mathbb{N}$ with $K \subseteq K_{n}$. In particular, $X=\bigcup_{n} K_{n}$ and $X$ is countable at infinity.

Lemma 6.7. Let $B \subseteq \mathcal{A}_{\mathbb{R}}^{\prime}$ be a weak-*-compact subset with Borel $\sigma$-algebra $\mathfrak{B}(B)$, and let $\left(\delta_{n}\right)$ be an approximate identity of $\mathcal{A}$ with $0 \leq \delta_{n} \leq 1$. For every spectral measure $P: \mathfrak{B}(B) \rightarrow B(\mathcal{H})$, we then have

$$
P\left(1^{*}\right)=s-\lim _{n \rightarrow \infty} P\left(\delta_{n}^{*}\right) .
$$

Proof. Since $B$ is weak-*-compact, it is weak-*-bounded, hence bounded by the uniform boundedness principle. Therefore, the sequence $\left(\delta_{n}^{*}\right)$ is uniformly bounded. Since $\delta_{n}^{*} \rightarrow 1^{*}$ pointwise by Lemma 6.5, the assertion follows from the standard continuity properties of spectral measures.

Proposition 6.8. Let $\mathfrak{k}$ be a compact Lie algebra and $\mathfrak{k}_{\mathcal{A}}:=C_{0}(X, \mathfrak{k})$ for a locally compact space $X$ countable at infinity. Then any bounded unitary representation $\pi: \mathfrak{k}_{\mathcal{A}} \rightarrow \mathfrak{u}(\mathcal{H})$ extends to the Banach-Lie algebra $\mathfrak{k}_{\mathcal{A}_{+}} \cong \mathfrak{k}_{\mathcal{A}} \rtimes \mathfrak{k}$ by

$$
\hat{\pi}(x):=s-\lim _{n \rightarrow \infty} \pi\left(x \otimes \delta_{n}\right) .
$$


Proof. For each $x \in \mathfrak{k}$, the map

$$
\pi_{X}: \mathcal{A}_{\mathbb{R}} \rightarrow \mathfrak{u}(\mathcal{H}), \quad a \mapsto \pi(x \otimes a)
$$

is a bounded representation of the abelian Lie algebra $\mathcal{A}_{\mathbb{R}}$. Hence, there exists a spectral measure $P_{X}$ on a weak-*-compact subset $B_{X} \subseteq \mathcal{A}_{\mathbb{R}}^{\prime}$ such that

$$
\pi_{X}(a)=i P_{X}(a)=i \int_{B_{X}} a d P_{X}
$$

[32, Theorem 4.1]. We now put

$$
\hat{\pi}(x):=i P_{X}\left(1^{*}\right)=s-\lim _{n \rightarrow \infty} i P_{X}\left(\delta_{n}\right) .
$$

Since the commutator bracket is separately strongly continuous on bounded subsets of $B(\mathcal{H})$, we obtain

$$
\begin{aligned}
{[\hat{\pi}(x), \pi(y \otimes a)] } & =s-\lim _{n \rightarrow \infty}\left[\pi\left(x \otimes \delta_{n}\right), \pi(y \otimes a)\right] \\
& =s-\lim _{n \rightarrow \infty} \pi\left([x, y] \otimes \delta_{n} a\right)=\pi([x, y] \otimes a)
\end{aligned}
$$

and

$$
[\hat{\pi}(x), \hat{\pi}(y)]=s-\lim _{n \rightarrow \infty}\left[\hat{\pi}(x), \pi\left(y \otimes \delta_{n}\right)\right]=s-\lim _{n \rightarrow \infty} \pi\left([x, y] \otimes \delta_{n}\right)=\hat{\pi}([x, y]) .
$$

Therefore,

$$
\hat{\pi}: \mathfrak{k}_{\mathcal{A}_{+}}=\mathfrak{k}_{\mathcal{A}} \rtimes \mathfrak{k} \rightarrow B(\mathcal{H}), \quad(x \otimes a, y) \mapsto \pi(x \otimes a)+\hat{\pi}(y)
$$

is a representation of the Banach-Lie algebra $\mathfrak{k}_{\mathcal{A}_{+}}$. Since $\mathfrak{k}$ is finite dimensional, it is also continuous.

Since every bounded unitary representation of $\mathfrak{k}_{\mathcal{A}}$ extends to $\mathfrak{k}_{\mathcal{A}_{+}}$, the classification of the irreducible bounded unitary representations of $\mathfrak{k}_{\mathcal{A}_{+}}$(Theorem 2.17) yields immediately the corresponding classification for $\mathfrak{k}_{\mathcal{A}}$ (cf. [34] for the case of unital commutative $C^{*}$-algebras).

Theorem 6.9. Let $\mathfrak{k}_{\mathcal{A}}=\mathfrak{k} \otimes_{\mathbb{R}} C_{0}(X, \mathbb{C})_{\mathbb{R}}$, with $\mathfrak{k}$ a compact semisimple Lie algebra, and $\mathcal{A}=C_{0}(X, \mathbb{C})$ the commutative $C^{*}$-algebra of continuous functions vanishing at infinity for a locally compact space $X$ countable at infinity. Then every bounded irreducible unitary representation $(\pi, \mathcal{H})$ of $\mathfrak{k}_{\mathcal{A}}$ is a finite tensor product of evaluation representations at different points corresponding to irreducible representations of $\mathfrak{k}$. In particular, $\mathcal{H}$ is finite dimensional. 


\subsection{Boundary conditions}

Let $X$ be a compact manifold with boundary $\partial X$ and let $\mathfrak{K} \rightarrow X$ be a Lie algebra bundle whose typical fiber is a compact semisimple Lie algebra $\mathfrak{k}$. We then denote by $\Gamma^{k}(\mathfrak{K})$ the Lie algebra of $C^{k}$-sections of $\mathfrak{K}$, and by

$$
\Gamma_{0}^{k}(\mathfrak{K}):=\left\{s \in \Gamma^{k}(\mathfrak{K}) ;\left.j^{k}(s)\right|_{\partial X}=0\right\}
$$

the subalgebra of sections whose derivatives of order $\leq k$ vanish at the boundary. The negative of the Killing form yields a smoothly varying inner product $\kappa: \mathfrak{K}_{X} \times \mathfrak{K}_{X} \rightarrow \mathbb{R}$ on the fibers. If we choose a metric $g$ on $X$ and a Lie connection $\nabla$ on $\mathfrak{K}$, both nondegenerate at $\partial X$, then $\nabla$ combines with the Levi-Civita connection to the covariant derivative $\nabla^{n}: \Gamma(\mathfrak{K}) \rightarrow \Gamma\left(\mathfrak{K} \otimes\left(T^{*} X\right)^{\otimes n}\right)$. The scalar product $\kappa \otimes g_{X}^{* \otimes n}$ induces the norm $\|\cdot\|_{g, X}$ on $\mathfrak{K}_{X} \otimes\left(T_{X}^{*} X\right)^{\otimes k}$, and we obtain the $C^{k}$-norm

$$
\|S\|_{k}:=\sup \left\{\sum_{j=0}^{k}\left\|\nabla^{j} S(x)\right\|_{g, x} ; \quad x \in X\right\}
$$

on $\Gamma^{k}(\mathfrak{K})$. This norm (multiplied by a suitable constant to guarantee $\left\|\left[s, s^{\prime}\right]\right\|_{k} \leq\|s\|_{k}\left\|s^{\prime}\right\|_{k}$ ) makes $\Gamma^{k}(\mathfrak{K})$ into a Banach Lie algebra with $\Gamma_{0}^{k}(\mathfrak{K})$ as a closed ideal. Note that different choices of $g$ and $\nabla$ yield equivalent norms.

Since the inclusion $\Gamma(\mathfrak{K}) \hookrightarrow \Gamma^{k}(\mathfrak{K})$ is continuous and dense, every irreducible bounded unitary representation of $\Gamma^{k}(\mathfrak{K})$ restricts to an irreducible bounded unitary representation of $\Gamma(\mathfrak{K})$, by which it is uniquely determined. By Theorem 4.11, these are finite tensor products of irreducible evaluation representations, hence extend to $\Gamma^{k}(\mathfrak{K})$. The Lie algebras $\Gamma(\mathfrak{K})$ and $\Gamma^{k}(\mathfrak{K})$ of smooth and $C^{k}$ sections thus have the same bounded irreducible representations, given by 4.11. The following theorem generalizes this observation to representations that need not be irreducible.

Theorem 6.10. If $X$ is compact, then any bounded unitary representation of $\Gamma(\mathfrak{K})$ extends to the Banach-Lie algebra $\Gamma^{0}(\mathfrak{K})$ of continuous sections of $\mathfrak{K}$ and even to the Banach-Lie algebra $\Gamma^{b}(\mathfrak{K})$ of all bounded sections.

Proof. Let $(\pi, \mathcal{H})$ be a bounded representation of $\Gamma(\mathfrak{K})$ and $\mathcal{A}:=C^{*}(\operatorname{im}(\pi))$ the $C^{*}$ algebra generated by its image. We have to show that the linear map $\pi: \Gamma(\mathfrak{K}) \rightarrow \mathcal{A}$ is continuous with respect to the norm $\|s\|_{\infty}:=\sup _{x \in X}\|s(x)\|$.

To this end, we recall that the irreducible representations of a $C^{*}$-algebra determine its norm [11, Theorem 2.7.3]. For every irreducible representation $\beta$ of $\mathcal{A}$, the 
unitary representation $\beta \circ \pi$ of $\Gamma(\mathfrak{K})$ is bounded and irreducible, hence a finite tensor product of evaluation representations $\pi_{x, \rho}$ (Theorem 4.11). We may therefore assume that $\pi$ is a direct sum of irreducible representations $\pi_{\mathbf{x}_{j}, \rho_{j}}, j \in J$. We now have to show that there exists a $C>0$ with

$$
\|\pi(s)\|=\sup _{j \in J}\left\|\pi_{\mathbf{x}_{j}, \rho_{j}}(s)\right\| \leq C\|s\|_{\infty} \quad \text { for } s \in \Gamma(\mathfrak{K}) .
$$

With $\mathbf{x}_{j}=\left\{x_{j}^{1}, \ldots, x_{j}^{n_{j}}\right\}$ and $\left\|\pi_{\mathbf{x}_{j}, \rho_{j}}\right\|=\sum_{i=1}^{n_{j}}\left\|\rho_{j}^{i}\right\|$, we thus obtain

$$
\|\pi(s)\|=\sup _{j \in J}\left\|\pi_{\mathbf{x}_{j}, \rho_{j}}(s)\right\| \leq\left(\sup _{j \in J} \sum_{i=1}^{n_{j}}\left\|\rho_{j}^{i}\right\|\right)\|s\|_{\infty} .
$$

It now remains to show that $C:=\sup _{j \in J} \sum_{i=1}^{n_{j}}\left\|\rho_{j}^{i}\right\|<\infty$. Since every summand $\pi_{\mathbf{x}_{j}, \rho_{j}}$ defines a bounded representation of $\Gamma^{0}(\mathfrak{K})$, it follows that $\pi$ extends to a continuous representation of the Lie algebra $\Gamma^{b}(\mathfrak{K})$ of all bounded sections.

Let $y_{0} \in X$ and pick open neighborhoods $V=V\left(y_{0}\right) \subseteq U$ of $y_{0}$ such that $\bar{V} \subseteq U$ is compact and $\left.\mathfrak{K}\right|_{U}$ is trivial. We identify $\Gamma_{C}\left(\left.\mathfrak{K}\right|_{U}\right) \subseteq \Gamma(\mathfrak{K})$ with $C_{C}^{\infty}(U, \mathfrak{k})$. Let $\chi \in C_{C}^{\infty}(U, \mathbb{R})$ with $0 \leq \chi \leq 1$ and $\left.\chi\right|_{V}=1$. We thus obtain a linear embedding $\gamma: \mathfrak{k} \rightarrow \Gamma_{C}\left(\left.\mathfrak{K}\right|_{U}\right) \subseteq \Gamma(\mathfrak{K}), x \mapsto$ $\chi x$. Since $\pi$ defines a bounded representation of $\Gamma(\mathfrak{K})$, the operator $\pi(\gamma(x))$ is bounded for every $x \in \mathfrak{k}$. In particular, we have

$$
\sup _{j \in J} \sum_{x_{j}^{i} \in V \cap \mathbf{x}_{j}}\left\|\rho_{j}^{i}(x)\right\|<\infty
$$

where we consider for $x_{j}^{i} \in V$ the representation $\rho_{j}^{i}$ of $\mathfrak{K}_{x_{j}^{i}}$ as a representation of $\mathfrak{k}$. As $\mathfrak{k}$ is finite dimensional, using these estimates for a linear basis of $\mathfrak{k}$, we obtain

$$
C_{Y_{0}}:=\sup _{j \in J} \sum_{x_{j}^{i} \in V \cap \mathbf{x}_{j}}\left\|\rho_{j}^{i}\right\|<\infty .
$$

Since $X$ is compact, there are finitely many points $y_{1}, \ldots, y_{N} \in X$ with $X \subseteq \bigcup_{k=1}^{N} V\left(y_{j}\right)$. Then $\left\|\pi_{\mathbf{x}_{j}, \rho_{j}}\right\| \leq \widetilde{C}:=\sum_{k} C_{Y_{k}}$ holds for every $j \in J$, and we thus obtain $\|\pi(s)\| \leq \widetilde{C}\left\|_{\infty}\right\|_{\infty}$.

This shows that, for all $k \geq 0$, the Banach-Lie algebra $\Gamma^{k}(\mathfrak{K})$ of $C^{k}$-sections has the same bounded unitary representation theory as the Fréchet-Lie algebra $\Gamma(\mathfrak{K})$ of smooth sections.

For the Lie algebra $\Gamma_{0}^{k}(\mathfrak{K})$, the situation is slightly more subtle, even in the case of irreducible representations. Although every bounded unitary factor (irreducible) representation of $\Gamma_{0}^{k}(\mathfrak{K})$ restricts to a bounded unitary factor (irreducible) representation of 
$\Gamma_{C}\left(\left.\mathfrak{K}\right|_{X^{\circ}}\right)$, the latter do not always extend to the former. The following theorem shows that bounded unitary factor (irreducible) representation of $\Gamma_{C}\left(\left.\mathfrak{K}\right|_{X^{\circ}}\right)$ (which are all obtained by infinite tensor products of irreducible evaluation representations, cf. Theorem 4.13), extend to bounded representations of $\Gamma_{0}^{k}(\mathfrak{K})$ only if the highest weights of the evaluation representations satisfy the following growth condition as the points approach the boundary.

Theorem 6.11. Let $X$ be a compact manifold with boundary, and $\mathfrak{K} \rightarrow X$ a Lie algebra bundle whose typical fiber is a compact simple Lie algebra. Then every factor (irreducible) bounded unitary representation of $\Gamma_{0}^{k}$ is equivalent to

$$
\pi_{\mathbf{x}, \rho, \beta}: \Gamma_{0}^{k}(\mathfrak{K}) \rightarrow B(\mathcal{H}), \quad s \mapsto \sum_{X \in \mathbf{X}} \beta \circ \iota_{X}\left(\rho_{X}(s(X))\right),
$$

where $(\mathbf{x}, \rho, \beta)$ is a triple with $\mathbf{x} \subset X^{\circ}$ a locally finite subset, $\rho=\left\{\left(\rho_{X}, V_{X}\right) ; x \in \mathbf{x}\right\}$ a set of irreducible representations of $\mathfrak{K}_{X}$ with highest weight $\lambda_{X}$ satisfying

$$
\sum_{x \in \mathbf{x}}\left\|\lambda_{x}\right\|_{\kappa} d(x, \partial X)^{k}<\infty
$$

and $(\beta, \mathcal{H})$ a factor (irreducible) representation of the $C^{*}$-algebra $\mathcal{A}_{\mathbf{x}, \rho}=\widehat{\bigotimes}_{\mathbf{x} \in \mathbf{x}} B\left(V_{X}\right)$ (cf. (6)).

In order to prove Theorem 6.11, we will have use for the following lemma.

Lemma 6.12. Let $\mathfrak{k}$ be a compact simple Lie algebra with invariant scalar product $\kappa$. Then there exists a constant $C(\mathfrak{k})>0$ such that

$$
C(\mathfrak{k})\|\lambda\|_{\kappa}\|x\|_{\kappa} \leq\|\rho(x)\| \leq\|\lambda\|_{\kappa}\|x\|_{\kappa}
$$

holds for all irreducible representations $\rho$ with highest weight $\lambda$, and for all $x \in \mathfrak{k}$.

Proof. Let $\mathfrak{t} \subseteq \mathfrak{k}$ be maximal abelian and $\mathfrak{h}:=\mathfrak{t}_{\mathbb{C}} \subseteq \mathfrak{g}:=\mathfrak{k}_{\mathbb{C}}$ the corresponding Cartan subalgebra (cf. Section 2). We also fix a connected Lie group $K$ with Lie algebra $\mathfrak{k}$. We denote by $\mathcal{W}:=N_{K}(\mathfrak{t}) / Z_{K}(\mathfrak{t})$ the Weyl group of $(\mathfrak{k}, \mathfrak{t})$, and by $\Delta^{+} \subseteq \Delta \subseteq \mathfrak{h}^{*}$ a positive system with respect to which $\lambda$ is the highest weight of $\rho$. Recall that every adjoint orbit $\operatorname{Ad}(K) X$ intersects

$$
\mathfrak{t}_{+}:=\left\{z \in \mathfrak{t}:\left(\forall \alpha \in \Delta^{+}\right) \mathrm{i} \alpha(z) \geq 0\right\},
$$

so we may assume that $x \in \mathfrak{t}_{+}$. 
First we recall that the set $\mathcal{P}_{\rho}$ of $\mathfrak{t}$-weights of $\rho$ is $\mathcal{W}$-invariant, contains $\lambda$ and is contained in $\operatorname{conv}(\mathcal{W} \lambda)$ (cf. [8, Chapter VIII]). As $\mathcal{W}$ acts isometrically on $\mathfrak{t}^{*}$ with respect to the induced norm, the relation

$$
\|\rho(x)\|=\sup |\langle\mathcal{W} \lambda, x\rangle| \leq\|\lambda\|_{\kappa}\|x\|_{\kappa}
$$

for $x \in \mathfrak{t}$ follows from the Cauchy-Schwarz inequality.

It remains to show that there exists a constant $C>0$ with $\|\rho(x)\| \geq C\|\lambda\|_{\kappa}$ for every $x \in \mathfrak{t}_{+}$with $\|x\|=1$. For $\beta \in \mathrm{it}^{*}$, let $t_{\beta} \in \mathfrak{t}$ be the unique element with $\kappa\left(t_{\beta}, z\right)=i \beta(z)$ for $z \in \mathfrak{t}$. Then $\mathfrak{t}_{+}=\left\{z \in \mathfrak{t}:\left(\forall \alpha \in \Delta^{+}\right) \kappa\left(t_{\alpha}, z\right) \geq 0\right\}$. For $\alpha \in \Delta$, we have $-i \check{\alpha}=\frac{2}{\left\|t_{\alpha}\right\|^{2}} t_{\alpha}$, so that $\kappa\left(t_{\lambda}, t_{\alpha}\right)=i \lambda\left(t_{\alpha}\right) \geq 0$ for $\alpha \in \Delta^{+}$implies $t_{\lambda} \in \mathfrak{t}_{+}$.

Now we observe that

$$
\|\rho(x)\| \geq|\lambda(x)|=\kappa\left(t_{\lambda}, x\right)
$$

so that it remains to show that

$$
\inf \left\{\kappa(y, z):\|y\|=\|z\|=1, y, z \in \mathfrak{t}_{+}\right\}>0 .
$$

By compactness, it suffices to show that $\kappa(y, z)>0$ for $0 \neq y, z \in \mathfrak{t}_{+}$. We argue by contradiction and assume that this is not the case. Then there exists non-zero $y, z \in \mathfrak{t}_{+}$with $\kappa(y, z) \leq 0$. Since

$$
\mathcal{W} z \subseteq z-\sum_{\alpha \in \Delta^{+}} \mathbb{R}_{+} t_{\alpha}
$$

by [30, Proposition V.2.7(ii)], we obtain $\kappa\left(y, z^{\prime}\right) \leq 0$ for $z^{\prime} \in \operatorname{conv}(\mathcal{W} z)$. The simplicity of $\mathfrak{k}$ implies that $\mathfrak{t}$ is a simple $\mathcal{W}$-module, so $f:=\sum_{w \in \mathcal{W}} w z$ is an element of $\mathfrak{t}^{\mathcal{W}}=\{0\}$. Since $\mathcal{W} z$ spans $\mathfrak{t}$, there exists a $w \in \mathcal{W}$ with $\kappa(w z, y) \neq 0$, hence $<0$, but this leads to the contradiction $0=\kappa(y, f)<0$.

Proof of Theorem 6.11. Since $X$ is compact, the continuous inclusion $\Gamma_{C}\left(\left.\mathfrak{K}\right|_{X^{\circ}}\right) \hookrightarrow \Gamma_{0}^{k}(\mathfrak{K})$ of Lie algebras is dense. Thus every norm continuous factor (irreducible) representation of $\Gamma_{0}^{k}(\mathfrak{K})$ restricts to a norm continuous factor (irreducible) representation of $\Gamma_{C}\left(\left.\mathfrak{K}\right|_{X^{\circ}}\right)$, and is uniquely defined by this restriction. Theorem 4.13 then yields a locally finite set $\mathbf{x} \subseteq X^{\circ}$ and representations $\rho_{X}$ of $\mathfrak{K}_{X}$ and $\beta$ of $\mathcal{A}_{\mathbf{x}, \rho}$ such that $\left.\pi\right|_{\Gamma_{c}\left(\left.\mathfrak{K}\right|_{X^{\circ}}\right)}=\beta \circ \eta_{\mathbf{x}, \rho}$. Since $\beta$ is an isometry, the representation of $\Gamma_{C}\left(\left.\mathfrak{K}\right|_{X^{\circ}}\right)$ extends to a bounded unitary representation of $\Gamma_{0}^{k}$ if and only if $\eta_{\mathbf{x}, \rho}: \Gamma_{C}\left(\left.\mathfrak{K}\right|_{X^{\circ}}\right) \rightarrow \mathcal{A}_{\mathbf{x}, \rho}$ is continuous w.r.t. the $C^{k}$-norm. In the following, we write $d_{x}:=d(x, \partial X)$ for brevity.

We first prove that $\sum_{x \in \mathbf{x}}\left\|\lambda_{x}\right\|_{\kappa} d_{x}^{k}<\infty$ implies continuity of $\eta_{\mathbf{x}, \rho}$. Recall that $\eta_{\mathbf{x}, \rho}(s):=\sum_{X \in \mathbf{x}} \iota_{X} \rho_{X}(s(X))$, where $\iota_{X}: B\left(V_{X}\right) \rightarrow \mathcal{A}_{\mathbf{x}, \rho}$ is the canonical inclusion. Since the $\iota_{X}$ 
are isometries, we have

$$
\left\|\eta_{\mathbf{x}, \rho}(s)\right\|=\sum_{x \in \mathbf{x}}\left\|\rho_{X}(s(x))\right\|
$$

By Lemma 6.12, we have the estimate $\left\|\rho_{X}(s(x))\right\| \leq\left\|\lambda_{X}\right\|_{\kappa}\|s(X)\|_{\kappa}$. Since every Lie connection is orthogonal w.r.t. $\kappa$, we can combine Taylor's theorem with the parallel transport equation to yield $\|s(x)\|_{\kappa} \leq \frac{1}{k !} d_{x}^{k}\|s\|_{k}$, and thus $\left\|\rho_{X}(s(x))\right\| \leq \frac{1}{k !}\left\|\lambda_{X}\right\|_{\kappa} d_{x}^{k}\|s\|_{k}$. Now (10) yields $\left\|\eta_{\mathbf{x}, \rho}(s)\right\| \leq \frac{1}{k !}\left(\sum_{X \in \mathbf{X}}\left\|\lambda_{X}\right\|_{\kappa} d_{x}^{k}\right)\|s\|_{k}$, which shows that $\eta_{\mathbf{x}, \rho}$ is continuous if $\sum_{X \in \mathbf{X}}\left\|\lambda_{X}\right\|_{\kappa} d_{X}^{k}<\infty$.

Conversely, suppose that $\sum_{x \in \mathbf{x}}\left\|\lambda_{X}\right\|_{\kappa} d_{x}^{k}=\infty$. We will exhibit sections $s_{\gamma}$ with $\left\|\eta_{\mathbf{x}, \rho}\left(s_{\gamma}\right)\right\| \geq 1$ but $\left\|s_{\gamma}\right\|_{k}$ arbitrarily small as $\gamma \downarrow 0$, showing that $\eta_{\mathbf{x}, \rho}$ is not continuous in the $C^{k}$-norm.

The first step is to localize the problem. Using the exponential flow for the metric $g$, we find an $\varepsilon \in\left(0, \frac{1}{2}\right)$, a finite covering of $\partial M$ by $U_{i} \subseteq \partial M$, open neighborhoods $V_{i} \supset \bar{U}_{i}$, and local diffeomorphisms $\phi_{i}: V_{i} \times[0,2 \varepsilon) \rightarrow X$, such that $\mathfrak{K}$ trivializes over $\operatorname{im}\left(\phi_{i}\right)$ and such that $d\left(\phi_{i}(v, x), \partial X\right)=x$ (cf. [45, Chapter 9, Theorem 20/21]). The local diffeomorphisms are defined by the flow $\phi_{i}(v, t)=\exp _{v}\left(t \vec{n}_{v}\right)$ along a (locally defined) inward pointing normal vector field $\vec{n}$ on $\partial M$.

Since $\mathbf{x}$ is locally finite in $X^{\circ}$, and since $X^{\circ}$ is covered by the open sets $\hat{U}_{i}:=$ $\phi_{i}\left(U_{i} \times(0, \varepsilon)\right)$ together with the compact set $\left\{x \in M ; d_{x} \geq \varepsilon / 2\right\} \subset X^{\circ}$, there is at least one $i$ such that $\sum_{x \in \mathbf{x} \cap \hat{U}_{i}}\left\|\lambda_{X}\right\|_{\kappa} d_{x}^{k}=\infty$.

Now let $\delta>0$ and let $\xi \in C_{c}^{\infty}\left(\operatorname{im}\left(\phi_{i}\right), \mathfrak{k}\right)$ be such that its restriction to $\hat{U}_{i}$ is a constant $\xi_{0} \in \mathfrak{k}$ with $\left\|\xi_{0}\right\|_{\kappa}=1$. Using the trivialization of $\mathfrak{K}$ over $\operatorname{im}\left(\phi_{i}\right)$, we define for every $\gamma \in(0,1)$ the section $s_{\gamma} \in \Gamma_{0}^{k}(X)$ by $s_{\gamma}\left(\phi_{i}(u, x)\right):=\delta x^{k+\gamma} \xi$. Since the topology induced by the $C^{k}$-norm is independent of the choice of connection, we may as well choose $\nabla$ to be compatible with the trivialization over $\operatorname{supp}(\xi)$. We then have $\left\|s_{\gamma}\right\|_{k} \leq \delta C\|\xi\|_{k}\left\|x^{k+\gamma}\right\|_{k}$ for some suitable constant $C$, with

$$
\left\|x^{k+\gamma}\right\|_{k}:=\sup \left\{\sum_{j=0}^{k}\left|\frac{d^{j}}{d x^{j}} x^{k+\gamma}\right|: x \in[0,2 \varepsilon]\right\} \leq \sum_{j=0}^{k+1} \frac{(k+1) !}{j !} \leq e(k+1) ! .
$$

Thus $\lim _{\delta \downarrow 0}\left\|s_{\gamma}\right\|_{k}=0$ uniformly in $\gamma$. However, we have $\left\|\rho_{X}(s(x))\right\| \geq C(\mathfrak{k})\left\|\lambda_{X}\right\|_{\kappa}\|s(x)\|_{\kappa}$ by Lemma 6.12, so that

$$
\left\|\eta_{\mathbf{x}, \rho}\left(s_{\gamma}\right)\right\| \geq \delta C(\mathfrak{k}) \sum_{x \in \mathbf{x} \cap \hat{U}_{i}}\left\|\lambda_{X}\right\|_{\kappa} d_{x}^{k+\gamma}
$$

Now since $\sum_{x \in \mathbf{x} \cap \hat{U}_{i}}\left\|\lambda_{x}\right\|_{\kappa} d_{x}^{k}=\infty$, there exists for every $N \in \mathbb{N}$ a finite subset $\mathbf{x}_{0} \subset \mathbf{x} \cap \hat{U}_{i}$ such that $\sum_{x \in \mathbf{x}_{0}}\left\|\lambda_{x}\right\|_{\kappa} d_{x}^{k}>N$. Taking the limit $\gamma \rightarrow 0$ in the inequality $\left\|\eta_{\mathbf{x}, \rho}(s)\right\| \geq \delta C(\mathfrak{k}) \sum_{x \in \mathbf{x}_{0}}\left\|\lambda_{X}\right\|_{\kappa} d_{x}^{k+\gamma}$ and choosing $N>1 /(\delta C(\mathfrak{k}))$, we see that there exists 
a $\gamma>0$ for which $\left\|\eta_{\mathbf{x}, \rho}\left(s_{\gamma}\right)\right\|>1$. Since $\lim _{\delta \downarrow 0}\left\|s_{\gamma}\right\|_{k}=0$ uniformly in $\gamma$, this finishes the proof.

The following is a direct consequence of Theorem 6.11 in the case $k=0$.

Corollary 6.13. Every bounded irreducible unitary representation $(\pi, \mathcal{H})$ of $\Gamma_{0}^{0}(\mathfrak{K})$ is a finite tensor product of irreducible evaluation representations. In particular, $\mathcal{H}$ is finite dimensional.

\subsection{Further problems}

It is an interesting question to which extent the theory developed in Sections 2 and 3 can be extended to the case where $\mathcal{A}$ is a general non-unital cia. Not unrelated, it would also be interesting to see whether the results on Lie algebras of sections in Section 4 extend to the context of complex analytic or algebraic geometry.

\subsubsection{Unital cias}

It is straightforward to apply our techniques to the unital cia $\mathcal{A}=\mathcal{O}_{\text {an }}\left(T^{n}\right)$ of analytic functions on the torus $T^{n} \subset \mathbb{C}^{n}$ (cf. Example 1.2(d)). The characters are given by evaluation in points of $T^{n}$, and are thus in particular involutive w.r.t. the involution $f^{*}\left(z_{1}, \ldots, z_{n}\right):=\bar{f}\left(1 / \bar{z}_{1}, \ldots, 1 / \bar{z}_{n}\right)$. Corollary 1.9 guarantees that every holomorphic multiplicative map $\phi: \mathcal{O}_{\text {an }}\left(T^{n}\right) \rightarrow \mathbb{C}$ is given by evaluation in finitely many points $p_{i} \in T^{n}$, that is, $\phi(f)=\Pi_{i=1}^{N} f\left(p_{i}\right)$. Using Theorem 2.11, we then see that every irreducible bounded *-representation of the Lie algebra $\mathcal{O}_{\text {an }}\left(T^{n}, \mathfrak{g}\right)$ of analytic functions on $T^{n}$ with values in $\mathfrak{g}=\mathfrak{k}_{\mathbb{C}}$, for $\mathfrak{k}$ a compact semisimple Lie algebra, is a tensor product of finitely many irreducible evaluation representations in different points $p_{i}$ of $T^{n}$.

\subsubsection{Non-unital cias}

If $\mathcal{A}$ is a non-unital cia, then the results of Sections 2 and 3 do not apply. The localization techniques of Section 4 will work for certain classes of cias which are soft sheaves over their spectra, but more 'rigid' non-unital cias, such as the algebra $\mathcal{A}_{p}=\mathcal{O}_{\text {an }}\left(T^{n}\right)_{p}$ of analytic functions on $T^{n}$ that vanish at $p \in T^{n}$, cannot be handled in this way.

It is clear that the Lie algebra $\mathfrak{g}\left(\mathcal{A}_{p}\right)$ allows for infinite tensor products of evaluation representations, coming from holomorphic multiplicative involutive maps of 
the form

$$
F: 1+\mathcal{A}_{p} \rightarrow \mathbb{C}, \quad(1+f) \mapsto \prod_{m=1}^{\infty}\left(1+f\left(p_{m}\right)\right),
$$

where $\left(p_{m}\right)_{m \in \mathbb{N}}$ is a sequence of points in $T^{n}$ with $\sum_{m=0}^{\infty} d\left(p_{m}, p\right)<\infty$ (cf. Examples 6.3 and 6.4). Here we use that, for any bounded set $S$ of holomorphic functions in a neighborhood $U$ of $T^{n}$, the set $\left\{f^{\prime}(p): f \in S\right\}$ of derivatives in $p$ is bounded by the Cauchy estimates.

In this connection, the following question arises: Suppose that $\mathcal{A}$ is a non-unital involutive cia, and $F: \mathbf{1}+\mathcal{A} \rightarrow \mathbb{C}$ a holomorphic, multiplicative and involutive map. Is the functional $\lambda:=\mathrm{d} F(\mathbf{1}): \mathcal{A} \rightarrow \mathbb{C}$ inducible? As the following proposition shows, it makes a serious difference whether we consider multiplicative homomorphisms on $(\mathcal{A}, \cdot)$ or on $(1+\mathcal{A}, \cdot)$.

Proposition 6.14. Let $\mathcal{A}$ be a non-unital commutative $\mathbf{k}$-algebra. Then every multiplicative map $\chi:(\mathcal{A}, \cdot) \rightarrow \mathbb{C}$ extends to a multiplicative map $\tilde{\chi}:\left(\mathcal{A}_{+}, \cdot\right) \rightarrow \mathbb{C}$ with $\tilde{\chi}(\mathbf{1})=1$. If, in addition, $\mathcal{A}$ is a complex complete cia and $\chi$ is holomorphic, then the same holds for $\tilde{\chi}$ and then $\chi$ is a finite product of algebra homomorphisms.

Proof. If $\chi=0$, then we put $\chi(a+t 1):=t$. We now assume that $\chi \neq 0$. For $a \in \mathcal{A}_{+}$, we write $\lambda_{a}: \mathcal{A} \rightarrow \mathcal{A}$ for the multiplication with $a$. Then there exists a $b \in \mathcal{A}$ with $\chi(b) \neq 0$. We put

$$
\chi_{b}(a):=\frac{\chi(a b)}{\chi(b)} \text { for } a \in \mathcal{A}_{+}, b \in \mathcal{A}
$$

For any other $c \in \mathcal{A}$ with $\chi(c) \neq 0$ and $a \in \mathcal{A}_{+}$, we then have

$$
\chi_{b}(a) \chi(b) \chi(c)=\chi(a b) \chi(c)=\chi(a b c)=\chi(a c b)=\chi(a c) \chi(b)=\chi_{c}(a) \chi(c) \chi(b)
$$

so that $\chi_{b}(a)=\chi_{c}(a)$. Therefore,

$$
\tilde{\chi} \mathcal{A}_{+} \rightarrow \mathbb{C}, \quad \tilde{\chi}(a):=\chi_{b}(a) \text { for } \chi(b) \neq 0
$$

is a well-defined extension of $\chi$. It satisfies $\chi(a b)=\tilde{\chi}(a) \chi(b)$ for $\chi(b) \neq 0$. If $\chi(b)=0$, then we choose a $c \in \mathcal{A}$ with $\chi(c) \neq 0$ and obtain

$$
\chi(a b) \chi(c)=\chi(a b c)=\chi(b a c)=\chi(b) \chi(a c)=0,
$$

so that $\chi(a b)=0$. This shows that

$$
\chi(a b)=\tilde{\chi}(a) \chi(b) \quad \text { for } a \in \mathcal{A}_{+}, b \in \mathcal{A} \text {. }
$$


For $a, a^{\prime} \in \mathcal{A}_{+}$and $\chi(b) \neq 0$, we then have

$$
\tilde{\chi}\left(a a^{\prime}\right)=\chi_{b}\left(a a^{\prime}\right)=\frac{\chi\left(a a^{\prime} b\right)}{\chi(b)}=\tilde{\chi}(a) \frac{\chi\left(a^{\prime} b\right)}{\chi(b)}=\tilde{\chi}(a) \tilde{\chi}\left(a^{\prime}\right) .
$$

Therefore, $\tilde{\chi}$ is multiplicative.

Now suppose that $\mathcal{A}$ is a complex complete cia and that $\chi$ is holomorphic. For any $b \in \mathcal{A}$ with $\chi(b) \neq 0$, the relation $\tilde{\chi}(a)=\chi_{b}(a)$, together with the holomorphy of the map $\mathcal{A}_{+} \rightarrow \mathcal{A}, a \mapsto a b$ now implies that also $\tilde{\chi}$ is holomorphic. We conclude with Theorem 1.8 that $\tilde{\chi}$ is a finite product of characters.

Not all bounded irreducible $*$-representations of $\mathcal{A}_{p}=\mathcal{O}_{\text {an }}\left(T^{n}\right)_{p}$ are highest weight representations. The Lie algebra $\mathfrak{s l}_{2}\left(\mathcal{A}_{p}\right)$ has an irreducible bounded $*$-representation,

$$
\pi(X):=\bigoplus_{m \in \mathbb{N}} \mathbf{1}^{m} \otimes X\left(p_{m}\right) \otimes 1^{\infty}
$$

on any infinite tensor product space $\mathcal{H}:=\bigotimes_{m \in \mathbb{N}}\left(\mathbb{C}^{2}, v_{m}\right)$, where $v_{m} \in \mathbb{C}^{2}$ is a sequence of unit vectors and $\left(p_{m}\right)_{m \in \mathbb{N}}$ a sequence of points in $T^{n}$ with $\sum_{m \in \mathbb{N}} d\left(p_{m}, p\right)<\infty$ (cf. (7) and Example 4.15). In particular, we have irreducible bounded unitary representations of $\mathfrak{s l}_{2}\left(\mathcal{A}_{p}\right)$ where $\mathcal{H}^{\mathfrak{g}^{-}}=\{0\}$.

Clearly, these cannot be classified with the highest weight theory from Section 2. This raises the following question: Suppose that $\mathcal{E}=\mathcal{H}^{\mathfrak{g}^{-}}$is non-zero for an irreducible bounded unitary representation of $\mathfrak{k}_{\mathcal{A}}$, with $\mathcal{A}$ a non-unital involutive complete cia. Then the arguments from the proof of Proposition 2.2 still imply that $\operatorname{dim} \mathcal{E}=1$, so that the representation $(\rho, \mathcal{E})$ of $\mathfrak{g}^{0}=h \otimes \mathcal{A}$ is given by a linear functional $\lambda: \mathcal{A} \rightarrow \mathbb{C}$. In view of the above example, $\lambda$ can be an infinite sum $\sum_{j=1}^{\infty} \lambda_{j} \otimes \chi_{j}$, so that Theorem 2.9 does not extend to the non-unital case. Does $F\left(\mathrm{e}^{a}\right):=\mathrm{e}^{\lambda(a)}$ extend to a multiplicative holomorphic $\operatorname{map} F: \mathcal{A} \rightarrow \mathbb{C}$ ?

\subsubsection{Multiloop algebras}

It would also be interesting to see whether the results on Lie algebras of sections in Section 4 extend to the context of complex analytic or algebraic geometry.

The class of twisted multiloop algebras is an interesting example. In this case, the finite group $H=\Pi_{i=1}^{n} \mathbb{Z} / k_{i} \mathbb{Z}$ acts on $\mathfrak{g}$ by automorphisms, and on $T^{n}$ by the free action $h \cdot z:=\left(\mathrm{e}^{2 \pi \mathrm{i} h_{1} / k_{1}} z_{1}, \ldots, \mathrm{e}^{2 \pi \mathrm{i} h_{n} / k_{n}} z_{n}\right)$. Then $\mathfrak{K}:=T^{n} \times_{H} \mathfrak{k}$ is a bundle of Lie algebras over $T^{n} / H \simeq T^{n}$, and the twisted multiloop algebras in the algebraic, analytic, and smooth 
setting are the corresponding Lie algebras of sections

$$
\mathcal{O}_{\text {alg }}\left(T^{n}, \mathfrak{g}\right)^{H} \subset \mathcal{O}_{\text {an }}\left(T^{n}, \mathfrak{g}\right)^{H} \subset C^{\infty}\left(T^{n}, \mathfrak{g}\right)^{H},
$$

where $\mathcal{O}_{\text {alg }}\left(T^{n}, \mathfrak{g}\right):=\mathbb{C}\left[t_{1}^{ \pm}, \ldots, t_{n}^{ \pm}\right] \otimes \mathfrak{g}$. Assuming that the action of $H$ on $\mathfrak{g}$ preserves a compact real form $\mathfrak{k}$, Theorem 4.11 implies that every irreducible bounded $*$-representation of $\Gamma(\mathfrak{K})_{\mathbb{C}} \simeq C^{\infty}\left(T^{n}, \mathfrak{g}\right)^{H}$ is a finite tensor product of irreducible evaluation representations in different points $p_{i}$ of $T^{n}$. Analogously, it follows from [28] that every finitedimensional irreducible representation of $\mathcal{O}_{\mathrm{alg}}\left(T^{n}, \mathfrak{g}\right)^{H}$ is given by a finite tensor product of evaluation representations, which implies the corresponding result in the analytic and smooth setting because the inclusions in (12) are dense. (See also [36], where the action of $H$ on $T^{n}$ is generalized to the automorphic action of a finite group on a scheme.)

This striking similarity raises the question whether our results extend to the analytic/algebraic setting also if the bundle $\mathfrak{K} \rightarrow X$ does not allow for a flat Lie connection, so that the structure group is not finite.

\section{Funding}

Partially supported by the Emerging Field Project "Quantum Geometry" of the FAU ErlangenNürnberg and by the NWO grant 613.001.214 "Generalised Lie algebra sheaves". Supported by DFG-grant NE 413/7-2, Schwerpunktprogramm “Darstellungstheorie".

\section{Acknowledgements}

We thank the referees for their thorough reading of the manuscript which lead to the elimination of several inaccuracies in the text. B. J. thanks the Mathematics Department of the FAU ErlangenNürnberg for hosting him during the time when this paper was written.

\section{References}

[1] Albeverio, S. and R. J. Høegh-Krohn. "The energy representation of Sobolev-Lie groups." Compositio Mathematica 36, no. 1 (1978): 37-51.

[2] Albeverio, S., R. J. Høegh-Krohn, J. A. Marion, D. H. Testard, and B. S. Torresani. Noncommutative Distributions - Unitary Representations of Gauge Groups and Algebras. Pure and Applied Mathematics 175. New York: Marcel Dekker, 1993.

[3] Ando, H. "On the local structure of the representation of a local gauge group." Infinite Dimensional Analysis, Quantum Probability and Related Topics 13, no. 2 (2010): 223-42.

[4] Araki, H. "Factorizable representation of current algebra- Non commutative extension of the Lévy-Kinchin formula and cohomology of a solvable group with values in a Hilbert Space." Publications of the Research Institute for Mathematical Sciences 5, no. 3 (1969): 361-422. 
[5] Arens, R. "Linear topological division algebras." Bulletin of the American Mathematical Society 53, no. 6 (1947): 623-30.

[6] Berezin, F. A. "Representations of the continuous direct product of universal coverings of the group of motions of the complex ball." Transactions of the Moscow Mathematical Society 1979, no. 2 (1979): 281-89.

[7] Biller, H. "Analyticity and naturality of the multi-variable functional calculus." Expositiones Mathematicae 25 (2007): 131-63.

[8] Bourbaki, N. Groupes et algèbres de Lie, Chapitres VII-VIII. Paris: Masson, 1990.

[9] Chari, V. and A. Pressley. "New unitary representations of loop groups." Mathematische Annalen 275, no. 1 (1986): 87-104.

[10] Chari, V. and A. Pressley. "Unitary representations of the maps $S^{1} \rightarrow s u(N, 1)$. " Mathematical Proceedings of the Cambridge Philosophical Society 102, no. 2 (1987): 259-72.

[11] Dixmier, J. $C^{*}$-Algebras. Amsterdam, New York, Oxford: North Holland Publishing Company, 1977.

[12] Dixmier, J. Les algèbres d'opérateurs dans l'espace Hilbertien. Éditions Gabay, 1996.

[13] Eyni, J. M. “Universal continuous bilinear forms for compactly supported sections of Lie algebra bundles and universal continuous extensions of certain current algebras." (2014): preprint arXiv:RA.1401.8154.

[14] Galina, E. and A. Kaplan. "On unitary representations of nilpotent gauge groups." Communications in Mathematical Physics 236, no. 2 (2003): 187-98.

[15] Gelfand, I. M. and M. I. Graev. "Representations of quaternion groups over locally compact fields and function fields." Funkcional'nyi Analiz I Ego Priloženija 2, no. 1 (1968): 20-35.

[16] Gelfand, I. M., M. I. Graev, and A. M. Vershik. "Representations of the group of functions taking values in a compact Lie group." Compositio Mathematica. 42, no. 2 (1980): 217-43.

[17] Glimm, J. "On a certain class of operator algebras." Transactions of the American Mathematical Society 95, no. 2 (1960): 318-40.

[18] Glöckner, H. "Algebras whose groups of units are Lie groups." Studia Mathematica. 153, no. 2 (2002): 147-77.

[19] Glöckner, H. "Lie groups over non-discrete topological fields." (2004): preprint arXiv:math/0408008 [math.GR].

[20] Guichardet, A. Symmetric Hilbert Spaces and Related Topics. Lecture Notes in Mathematics. 261. Springer, 1972.

[21] Henrichs, R. W. "Decomposition of invariant states and nonseparable $C^{*}$-algebras." Publications of the Research Institute for Mathematical Sciences, Kyoto University. 18, no. 1 (1982): 159-81.

[22] Ismagilov, R. S. "Unitary representations of the group $C_{C}^{\infty}(X, G), G=\mathrm{SU}_{2}$." Matematicheskii Sbornik (N.S.) 100(142), no. 1(5) (1976): 117-31, 166.

[23] Ismagilov, R. S. Representations of Infinite-Dimensional Groups. Translations of Mathematical Monographs 152. American Mathematical Society, 1996.

[24] Jakobsen, H. P. and V. Kac. "A New Class of Unitarizable Highest Weight Representations of Infinite-Dimensional Lie Algebras." In Non-Linear Equations in Classical and Quantum 
Field Theory, edited by N. Sanchez, 1-20. Lecture Notes in Physics 226. Berlin, Heidelberg, New York: Springer, 1985.

[25] Jakobsen, H. P. and V. Kac. "A new class of unitarizable highest weight representationsof infinite-dimensional Lie algebras, II." Journal of Functional Analysis 82, no. 1 (1989): 69-90.

[26] Janssens, B. and K.-H. Neeb. "Projective unitary representations extending bounded factor representations of mapping Lie algebras." in preparation

[27] Janssens, B. and C. Wockel. "Universal central extensions of gauge algebras and groups." Journal für die Reine und Angewandte Mathematik 682 (2013): 129-39.

[28] Lau, M. “Representations of multiloop algebras." Pacific Journal of Mathematics. 245, no. 1 (2010): 167-84.

[29] Maier, P. "Central Extensions of Topological Current Algebras." In Geometry and Analysis on Finite- and Infinite-Dimensional Lie Groups, edited by A. Strasburger et al. 61-76. Banach Center Publications 55. Warszawa, 2002.

[30] Neeb, K.-H. Holomorphy and Convexity in Lie Theory. Expositions in Mathematics 28. Berlin: de Gruyter Verlag, 2000.

[31] Neeb, K.-H. "Towards a Lie theory of locally convex groups." Japanese Journal of Mathematics 3rd series. 1, no. 2 (2006): 291-468.

[32] Neeb, K.-H. "Semibounded Unitary Representations of Infinite Dimensional Lie Groups." In Infinite Dimensional Harmonic Analysis IV, edited by J. Hilgert et al., 209-22. World Scientific, 2009.

[33] Neeb, K.-H. "Positive energy representations and continuity of projective representations for general topological groups." Glasgow Mathematical Journal 56 (2014): 295-316.

[34] Neeb, K.-H. and H. Seppänen. "Borel-Weil theory for groups over commutative Banach algebras." Journal für die reine und angewandte Mathematik. 655 (2011): 165-87.

[35] Neher, E. and A. Savage. "A Survey of Equivariant Map Algebras with Open Problems." In Recent Developments in Algebraic and Combinatorial Aspects of Representation Theory (Bangalore, India, 2010 and Riverside, CA, 2012), 165-82. Contemporary Mathematics 602. Providence, RI: American Mathematical Society, 2013.

[36] Neher, E., A. Savage, and P. Senesi. "Irreducible finite-dimensional representations of equivariant map algebras." Transactions of the American Mathematical Society 364, no. 5 (2012): 2619-46.

[37] Olshanski, G. I. "Spherical functions and characters of the group $G^{X}$." Russian Mathematical Surveys 37, no. 2 (1982): 233-4.

[38] Powers, R. T. "Representations of uniformly hyperfinite algebras and their associated von Neumann algebras." Annals of Mathematics 86, no. 1 (1967): 138-71.

[39] Pressley, A. and G. Segal. Loop Groups. Oxford: Oxford University Press, 1986.

[40] Rudin, W. Real and Complex Analysis. New York: McGraw Hill, 1986.

[41] Rudin, W. Functional Analysis. International Series in Pure and Applied Mathematics. New York: McGraw-Hill, 1991.

[42] Sakai, S. "On type I $C^{*}$-algebras." Proceedings of the American Mathematical Society 18, no. 5 (1967): 861-63. 
[43] Sakai, S. $C^{*}$-Algebras and $W^{*}$-Algebras. Ergebnisse der Math. und ihrer Grenzgebiete 60. Berlin, Heidelberg, New York: Springer, 1971.

[44] Samoilenko, Y. S. Spectral Theory of Families of Self-Adjoint Operators. Mathematics and Its Applications (Soviet Series). Dordrecht: Kluwer, 1991.

[45] Spivak, M. A Comprehensive Introduction to Differential Geometry. Vol. 1, Boston, MA: Publish or Perish, 1970.

[46] Takesaki, M. Theory of Operator Algebras. Encyclopedia of Mathematical Sciences 125. Operator Algebras and Non-commutative Geometry 6. Berlin: Springer, 2003.

[47] Thiemann, Th. Modern Canonical Quantum General Relativity. Cambridge Monographs on Mathematical Physics, 2008.

[48] Torresani, B. S. "Unitary positive energy representations of the gauge group." Letters in Mathematical Physics 13, no. 1 (1987): 7-15.

[49] Treves, F. Topological Vector Spaces, Distributions and Kernels. San Diego: Academic Press, 1967.

[50] Turpin, Ph. "Une remarque sur les algèbres à inverse continue." Comptes rendus de l'Académie des sciences, Paris, Tome 270, Série A (1970): A1686-9.

[51] Vershik, A. M., I. M. Gelfand, and M. I. Graev. "Irreducible representations of the group $G^{X}$ and cohomology." Functional Analysis and Its Applications . 8, no. 2 (1974): 67-9.

[52] Waelbroeck, L. "Le calcul symbolique dans les algèbres commutatives." Journal de Mathématiques Pures et Appliquées 33, no. 9 (1954): 147-86.

[53] Wagner, S. "Extending characters on fixed point algebras." (2011): preprint arXiv:1111.5560 [math.DS].

[54] Wagner, S. "A geometric approach to noncommutative principal torus bundles." Ph.D. thesis, FAU Erlangen-Nuremberg, 2011; arXiv:1108.4294 [math.DG].

[55] Wockel, C. "Smooth extensions and spaces of smooth and holomorphic mappings." Journal of Geometry and Symmetry in Physics 5 (2006): 126-34; see also arXiv:0511064 [math.DG] 